\title{
Diastereoselective Cobalt-Mediated Cross-Couplings of Cycloalkyl lodides with Alkynyl or (Hetero)Aryl \\ Grignard Reagents
}

Jeffrey M. Hammann, Diana Haas, Carl-Phillip Tüllmann, Konstantin Karaghiosoff and Paul Knochel*

\author{
Ludwig-Maximilians-University Munich \\ Department of Chemistry
}

Butenandtstr. 5-13, 81377 Munich, Germany

Paul.Knochel@cup.uni-muenchen.de

\section{Supporting Information}

\section{Table of Content}

General Information

Diastereoselective Cobalt-Mediated Cross-Couplings of Cycloalkyl lodides with Alkynyl 


\section{General Information}

Unless otherwise indicated, all reactions were carried out with magnetic stirring and in flame-dried glassware under argon. Syringes used to transfer reagents and solvents were purged with argon prior to use. Reactions were monitored by gas chromatography (GC and GC-MS) or thin layer chromatography (TLC). TLCS were performed using aluminum plates covered with $\mathrm{SiO}_{2}$ (Merck 60, F-254) and visualized by UV detection. Purification via column chromatography was performed using Merck silica gel 60 (40-63 mm 230-400 mesh ASTM from Merck). THF was continuously refluxed and freshly distilled from sodium benzophenone ketyl under nitrogen. Melting points were measured using a Büchi B-540 apparatus and are uncorrected. NMR spectra were recorded in $\mathrm{CDCl}_{3}$ and chemical shifts $(\delta)$ are reported in parts per million (ppm). Mass spectra and highresolution mass spectra (HR-MS) were recorded using electro ionization (EI) except where otherwise noted. GCs were recorded on machines of the type Hewlett-Packard 6890 (Hewlett Packard, 5\% phenylmethylpolysiloxane; length: $15 \mathrm{~m}$, diameter: $0.25 \mathrm{~mm}$; film thickness: $0.25 \mu \mathrm{m}) . \mathrm{CoCl}_{2}$ (97\% purity) was purchased from Fisher Scientific.

\section{Optimization of the Reaction Conditions: Solvent Screening}

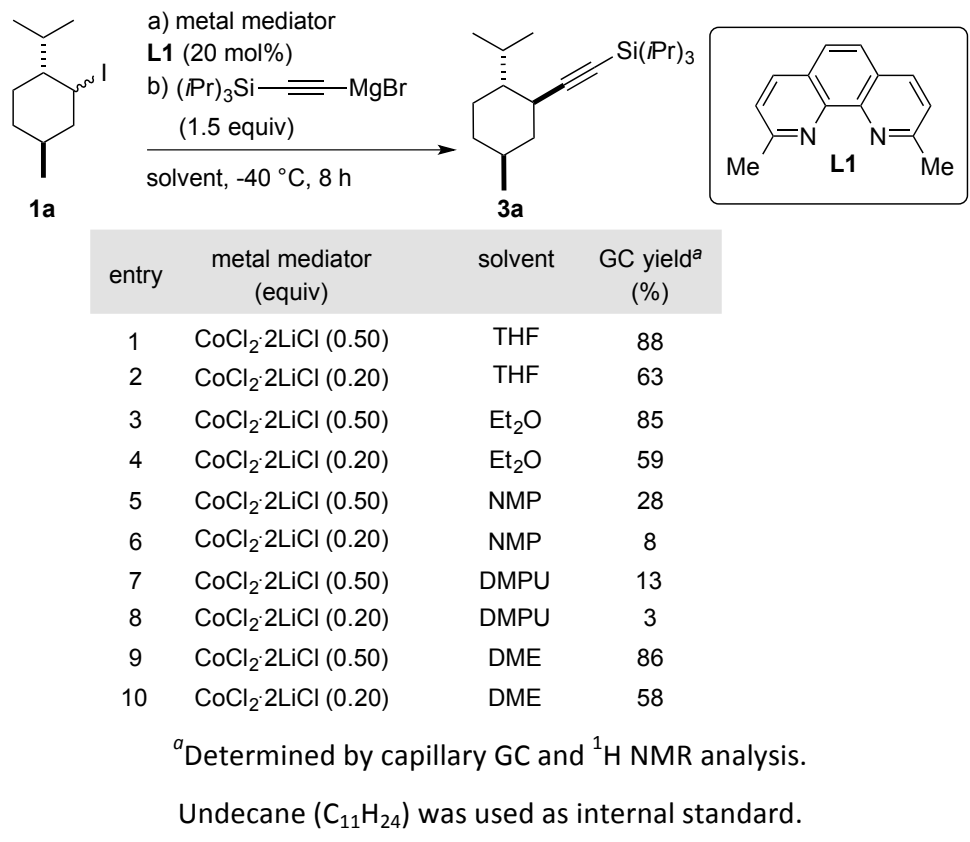

Noteworthy is, that all reactions gave the desired cross-coupling product with a dr of 99:1.

\section{Preparation of Organometallic Reagents}

\section{Preparation of $\mathrm{CoCl}_{2} \cdot \mathbf{2} \mathrm{LiCl}(1.0 \mathrm{M})$ :}

A dry and argon-flushed $250 \mathrm{~mL}$ Schlenk-flask, equipped with a stirring bar and a septum, was charged with anhydrous $\mathrm{LiCl}\left(200 \mathrm{mmol}, 8.48 \mathrm{~g}\right.$ ) and heated to $130^{\circ} \mathrm{C}$ under high vacuum for $5 \mathrm{~h}$. After cooling to r.t. under 
argon, anhydrous $\mathrm{CoCl}_{2}(100 \mathrm{mmol}, 12.98 \mathrm{~g})$ was added. The Schlenk-flask was further heated to $130{ }^{\circ} \mathrm{C}$ for $5 \mathrm{~h}$ under high vacuum, cooled to $25^{\circ} \mathrm{C}$ and charged with dry THF $(100 \mathrm{~mL})$. The mixture was vigorously stirred until all solids were dissolved (ca. $8 \mathrm{~h}$ ). The reagent $\mathrm{CoCl}_{2} \cdot 2 \mathrm{LiCl}(1 \mathrm{M}$ in THF) is obtained as a dark blue solution.

\section{Preparation of EtMgBr (0.94 M):}

A dry and argon-flushed $100 \mathrm{~mL}$ Schlenk-flask, equipped with a stirring bar and a septum, was charged with magnesium turnings (60 mmol, $1.44 \mathrm{~g}$ ) in THF $(50 \mathrm{~mL})$. Bromoethane $(50 \mathrm{mmol}, 5.4 \mathrm{~g})$ was added dropwise at $-20^{\circ} \mathrm{C}$ and the reaction mixture was shortly heated to reflux and again cooled to $-20^{\circ} \mathrm{C}$. This procedure was repeated until the reaction started. After disappearance of the Mg turnings the dark-grey solution was titrated by using a stoichiometric amount of iodine $(100 \mathrm{mg})$ in THF $(2 \mathrm{~mL})$ and a concentration of $0.94 \mathrm{M}$ was determined.

\section{Preparation of TMPMgCl-LiCl (1.15 M):}

A dried and argon-flushed 2 L Schlenk-flask, equipped with a magnetic stirring bar and rubber septum, was charged with iPrMgCl. LiCl (1.31 M in THF, $850 \mathrm{~mL}, 1.11 \mathrm{~mol})$. Then, 2,2,6,6-tetramethylpiperidine (161 g, 194 $\mathrm{mL}, 1.14 \mathrm{~mol}, 1.02$ equiv) was added at once and the mixture was stirred until gas evolution ceases (48 h). Titration with benzoic acid using 4-(phenylazo)diphenylamine as indicator prior to use showed a concentration of about $1.15 \mathrm{M}$.

\section{Preparation of alkynyl grignard reagents:}

The corresponding alkyne (1 equiv, $1 \mathrm{mmol})$ and THF $(1 \mathrm{~mL})$ were added to a dry and argon-flushed $10 \mathrm{~mL}$ Schlenk-flask, equipped with a stirring bar and a septum. $\mathrm{TMPMgCl} \cdot \mathrm{LiCl}$ (1.2 equiv, addition at $0{ }^{\circ} \mathrm{C}$ ) or $\mathrm{EtMgBr}$ (1.2 equiv, addition at $-20^{\circ} \mathrm{C}$ ) were added dropwise and the reaction mixture was allowed to warm to room temperature and stirred until a reaction aliquot quenched with $\mathrm{I}_{2}$ in THF showed full conversion of the starting material by GC.

\section{Preparation of aryl magnesium reagents via insertion of magnesium:}

$\mathrm{LiCl}$ (1.1 equiv) was dried under high vacuum and allowed to cool to room temperature, then $\mathrm{Mg}$ turnings (1.2 equiv) and THF ( $1 \mathrm{M}$ solution relating to the aryl bromide) were added. The reaction mixture was cooled to $0{ }^{\circ} \mathrm{C}$ and the corresponding aryl bromide (1.0 equiv) was added dropwise. The reaction was stirred until iodolysis of a reaction aliquot indicated full consumption of the starting material.

\section{Preparation of aryl magnesium reagents via iodine-magnesium exchange:}

The corresponding aryl bromide (1.0 equiv) was dissolved in THF ( $1 \mathrm{M}$ solution relating to the aryl bromide) and the reaction mixture was cooled to $-30^{\circ} \mathrm{C}$. Then $i-\mathrm{PrMgCl} \cdot \mathrm{LiCl}$ (1.1 equiv) was added dropwise and the reaction was stirred at this temperature until reaction aliquots quenched with iodine showed full consumption of the starting material. 


\section{Synthesis of Starting Materials}

The following starting materials were prepared according to literature procedures with only little deviation. The spectral data of knowncompounds are in full agreement with the literature.

2-lodo-1-isopropyl-4-methylcyclohexane (1a) ${ }^{1}$<smiles>CC(C)[C@H]1CC[C@@H](C)C[C@H]1I</smiles>

$d r=99: 1$

${ }^{1} \mathrm{H}-\mathrm{NMR}\left(300 \mathrm{MHz}, \mathrm{CDCl}_{3}, \mathrm{ppm}\right): \delta=4.94(\mathrm{p}, J=2.8 \mathrm{~Hz}, 1 \mathrm{H}), 2.38$ (dtd, $\left.J=14.5,3.3,2.2 \mathrm{~Hz}, 1 \mathrm{H}\right), 2.14$ (tdd, $J=$ 11.6, 5.7, 3.2 Hz, 1H), $1.92(\mathrm{ttd}, J=12.7,3.5,2.2 \mathrm{~Hz}, 2 \mathrm{H}), 1.58-1.36(\mathrm{~m}, 3 \mathrm{H}), 1.15-1.01(\mathrm{~m}, 10 \mathrm{H}), 0.00(\mathrm{ddt}, J=$ 11.9, 9.1, $2.9 \mathrm{~Hz}, 1 \mathrm{H})$.

tert-Butyl((2-iodocyclopentyl)oxy)dimethylsilane (1b) ${ }^{2}$

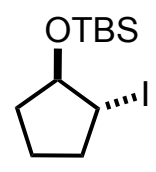

$\mathbf{d r}=99: 1$

${ }^{1} \mathrm{H}-\mathrm{NMR}\left(300 \mathrm{MHz}^{\mathrm{CDCl}}{ }_{3}, \mathrm{ppm}\right): \delta=4.43(\mathrm{ddd}, J=6.3,3.6,3.3 \mathrm{~Hz}, 1 \mathrm{H}), 4.06-3.98(\mathrm{~m}, 1 \mathrm{H}), 2.41-2.28(\mathrm{~m}, 1 \mathrm{H})$, $2.16-1.97(\mathrm{~m}, 2 \mathrm{H}), 1.80$ (quin, $J=7.4 \mathrm{~Hz}, 2 \mathrm{H}$ ), $1.60-1.49(\mathrm{~m}, 1 \mathrm{H}), 0.89(\mathrm{~s}, 9 \mathrm{H}), 0.10(\mathrm{~s}, 3 \mathrm{H}), 0.07(\mathrm{~s}, 3 \mathrm{H})$.

2-(2-lodocyclohexyl)-5,5-dimethyl-1,3-dioxane (1c) $)^{3}$

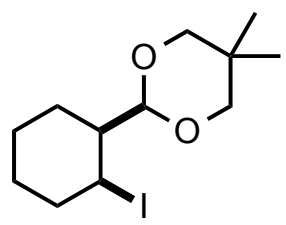

\footnotetext{
${ }^{1}$ S. D. Lepore, D. Mondal, S. Y. Li, A. K. Bhunia, Angew. Chem. Int. Ed. 2008, 47, 7511.

2 M. R. Detty, M. D. Seidler, J. Org. Chem. 1981, 46, 1283.

${ }^{3}$ K. Moriya, P. Knochel, Org. Lett. 2014, 16, 924.
} 
$d r=99: 1$

${ }^{1} \mathrm{H}-\mathrm{NMR}\left(300 \mathrm{MHz}, \mathrm{CDCl}_{3}, \mathrm{ppm}\right): \delta=4.85(\mathrm{dq}, J=3.1,1.7 \mathrm{~Hz}, 1 \mathrm{H}), 4.07$ (d, $\left.J=7.5 \mathrm{~Hz}, 1 \mathrm{H}\right), 3.60$ (ddd, $J=11.6$, 9.3, $2.7 \mathrm{~Hz}, 2 \mathrm{H}), 3.43(\mathrm{dd}, J=11.4,5.0 \mathrm{~Hz}, 2 \mathrm{H}), 2.19-2.13(\mathrm{~m}, 1 \mathrm{H}), 1.79-1.62(\mathrm{~m}, 4 \mathrm{H}), 1.55(\mathrm{~s}, 1 \mathrm{H}), 1.40-1.22$ (m, 2H), $1.17(\mathrm{~s}, 3 \mathrm{H}), 0.77(\mathrm{ddt}, J=10.6,7.6,2.9 \mathrm{~Hz}, 1 \mathrm{H}), 0.72(\mathrm{~s}, 3 \mathrm{H})$.

2-lodo-4-methyl-1-(prop-1-en-2-yl)cyclohexane (1d) ${ }^{1}$

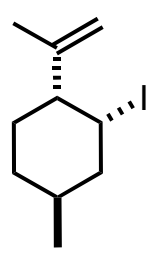

$\mathbf{d r}=99: 1$

${ }^{1} \mathrm{H}-\mathrm{NMR}\left(300 \mathrm{MHz}^{\mathrm{CDCl}} \mathrm{CD}_{3}, \mathrm{ppm}\right): \delta=4.86(\mathrm{ddd}, J=5.8,3.0,1.5 \mathrm{~Hz}, 2 \mathrm{H}), 4.66(\mathrm{td}, J=1.7,0.8 \mathrm{~Hz}, 1 \mathrm{H}), 2.25(\mathrm{dtd}, J$ $=14.4,3.2,2.3 \mathrm{~Hz}, 1 \mathrm{H}), 1.99(\mathrm{tdt}, J=14.8,6.6,3.1 \mathrm{~Hz}, 1 \mathrm{H}), 1.87-1.75(\mathrm{~m}, 1 \mathrm{H}), 1.74(\mathrm{dt}, J=1.4,0.7 \mathrm{~Hz}, 3 \mathrm{H}), 1.70$ $-1.53(\mathrm{~m}, 2 \mathrm{H}), 1.45(\mathrm{ddd}, J=14.6,11.5,3.3 \mathrm{~Hz}, 1 \mathrm{H}), 1.30-1.15(\mathrm{~m}, 1 \mathrm{H}), 1.05(\mathrm{dtd}, J=13.3,12.0,4.8 \mathrm{~Hz}, 1 \mathrm{H})$, $0.94(d, J=6.6 \mathrm{~Hz}, 3 \mathrm{H})$.

tert-Butyl((2-iodocyclohexyl)oxy)dimethylsilane ${ }^{4}$

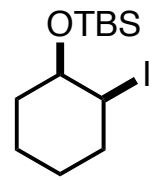

$d r=25: 75$

${ }^{1} \mathrm{H}-\mathrm{NMR}\left(300 \mathrm{MHz}^{\mathrm{CDCl}} \mathrm{CD}_{3} \mathrm{ppm}\right): \delta=4.53-4.39(\mathrm{~m}, 1 \mathrm{H}), 3.36$ (s br., $\left.1 \mathrm{H}\right), 2.32-2.20(\mathrm{~m}, 1 \mathrm{H}), 1.96-1.84(\mathrm{~m}, 1$ H), $1.79-1.60(\mathrm{~m}, 3 \mathrm{H}), 1.57-1.30(\mathrm{~m}, 3 \mathrm{H}), 0.95(\mathrm{~s}, 9 \mathrm{H}), 0.14(\mathrm{~s}, 3 \mathrm{H}), 0.08(\mathrm{~s}, 3 \mathrm{H})$.

\footnotetext{
${ }^{4}$ M. Smietana, V. Gouverneur, C. Mioskowski, Tetrahedron Lett. 2000, 41, 193.
} 


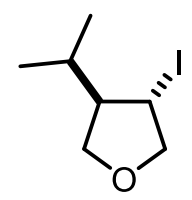

$d r=99: 1$

${ }^{1} \mathrm{H}-\mathrm{NMR}\left(400 \mathrm{MHz} \mathrm{CDCl}_{3}, \mathrm{ppm}\right): \delta=4.21-4.12(\mathrm{~m}, 1 \mathrm{H}), 3.81-3.67(\mathrm{~m}, 2 \mathrm{H}), 3.43(\mathrm{t}, J=7.9 \mathrm{~Hz}, 1 \mathrm{H}), 1.94(\mathrm{~d}, J=$ $5.8 \mathrm{~Hz}, 1 \mathrm{H}$ ), 1.80 (ddd, $J=10.9,8.8,5.5 \mathrm{~Hz}, 1 \mathrm{H}), 1.57$ (dh, $J=13.5,6.6 \mathrm{~Hz}, 1 \mathrm{H}), 1.02(\mathrm{~d}, J=6.6 \mathrm{~Hz}, 3 \mathrm{H}), 0.89(\mathrm{~d}$, $J=6.7 \mathrm{~Hz}, 3 \mathrm{H})$.

3-lodo-4-(prop-1-en-2-yl)tetrahydrofuran

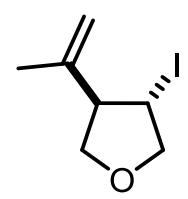

$d r=99: 1$

${ }^{1} \mathrm{H}-\mathrm{NMR}\left(300 \mathrm{MHz}^{\mathrm{CDCl}}\right.$, ppm): $\delta=5.03-4.91(\mathrm{~m}, 1 \mathrm{H}), 4.62-4.60(\mathrm{~m}, 1 \mathrm{H}), 4.57(\mathrm{td}, J=4.6,1.6 \mathrm{~Hz}, 1 \mathrm{H}), 4.52$ (dd, $J=10.4,4.4 \mathrm{~Hz}, 1 \mathrm{H}), 4.41(\mathrm{dd}, J=10.4,1.6 \mathrm{~Hz}, 1 \mathrm{H}), 4.03(\mathrm{~s}, 1 \mathrm{H}), 4.01(\mathrm{~d}, J=1.8 \mathrm{~Hz}, 1 \mathrm{H}), 2.34-2.27(\mathrm{~m}, 1 \mathrm{H})$, $1.79(\mathrm{dt}, J=1.6,0.8 \mathrm{~Hz}, 3 \mathrm{H})$.

3-lodo-4-isopropyl-1-tosylpyrrolidine

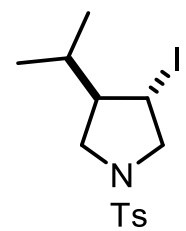

$d r=99: 1$

${ }^{1} \mathrm{H}-\mathrm{NMR}\left(300 \mathrm{MHz}^{\mathrm{CDCl}} \mathrm{CDpm}_{3}\right.$ ): $\delta=7.74(\mathrm{~d}, J=7.9 \mathrm{~Hz}, 2 \mathrm{H}), 7.33(\mathrm{~d}, J=7.9 \mathrm{~Hz}, 2 \mathrm{H}), 4.33(\mathrm{t}, J=4.1 \mathrm{~Hz}, 1 \mathrm{H}), 4.16$ (dd, $J=12.4,4.3 \mathrm{~Hz}, 1 \mathrm{H}), 3.97(\mathrm{~d}, J=12.4 \mathrm{~Hz}, 1 \mathrm{H}), 3.56(\mathrm{dd}, J=11.1,9.3 \mathrm{~Hz} \mathrm{~Hz}, 1 \mathrm{H}), 3.01(\mathrm{dd}, J=11.0,9.3 \mathrm{~Hz}$, 
$1 \mathrm{H}), 2.43(\mathrm{~s}, 3 \mathrm{H}), 1.43(\mathrm{dp}, J=9.5,6.6 \mathrm{~Hz}, 1 \mathrm{H}), 0.92(\mathrm{~d}, J=6.6 \mathrm{~Hz}, 3 \mathrm{H}), 0.82(\mathrm{~d}, J=6.5 \mathrm{~Hz}, 3 \mathrm{H}), 0.78-0.70(\mathrm{~m}$, $1 \mathrm{H})$. 


\section{Diastereoselective Cobalt-Mediated Cross-Couplings of Cycloalkyl lodides with Alkynyl or (Hetero)Aryl Grignard Reagents}

\section{Typical procedure (TP) for the cobalt-mediated cross-coupling reaction:}

A dry and argon-flushed $10 \mathrm{~mL}$ Schlenk-tube was charged with $\mathrm{CoCl}_{2} \cdot \mathrm{LiCl}(1.0 \mathrm{M}$ in THF) $(0.25 \mathrm{mmol}, 0.25 \mathrm{~mL}$, $50 \mathrm{~mol} \%)$ and neocuproine $(0.2 \mathrm{mmol}, 21 \mathrm{mg}, 20 \mathrm{~mol} \%)$, in dry THF $(1 \mathrm{~mL})$. The respective cycloalkyl iodide $(0.5 \mathrm{mmol})$ was added and the mixture was cooled to $-40{ }^{\circ} \mathrm{C}$ or $0{ }^{\circ} \mathrm{C}$. Then, a solution of the appropriate alkynyl or aryl Grignard reagent ( $0.75 \mathrm{mmol}, 1.5$ equiv) was added dropwise over $10 \mathrm{~min}$ via syringe. The reaction was stirred and monitored by GC-analysis $\left(\mathrm{C}_{14} \mathrm{H}_{30}\right.$ was used as an internal standard). Upon consumption of the starting material, saturated aq. $\mathrm{NH}_{4} \mathrm{Cl}$ solution $(5 \mathrm{~mL})$ was added, the phases were separated and the aqueous phase was extracted with EtOAc $(3 \times 25 \mathrm{~mL})$. The combined organic layers were dried over $\mathrm{MgSO}_{4}$. The solvents were evaporated and the residue was subjected to column chromatography on silica yielding the respective title compound.

Triisopropyl(((1R,2S,5R)-5-methyl-2-(prop-1-en-2-yl)cyclohexyl)ethynyl)silane (3a)

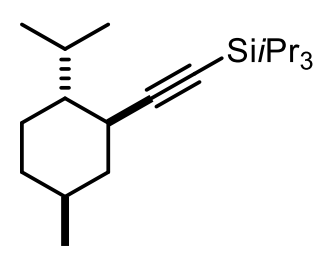

Isolated yield: $108 \mathrm{mg}, 0.34 \mathrm{mmol}, 68 \%$, colorless oil

dr: 99:1.

Purification: $i$-hexane

${ }^{1} \mathrm{H}-\mathrm{NMR}\left(300 \mathrm{MHz} \mathrm{CDCl}_{3}, \mathrm{ppm}\right): \delta=2.27$ (ddq, $\left.J=9.9,7.0,3.5,2.9 \mathrm{~Hz}, 1 \mathrm{H}\right), 2.11(\mathrm{td}, J=11.5,3.5 \mathrm{~Hz}, 1 \mathrm{H}), 1.92$ $(\mathrm{dq}, J=12.9,3.4 \mathrm{~Hz}, 1 \mathrm{H}), 1.67-1.59(\mathrm{~m}, 1 \mathrm{H}), 1.59-1.49(\mathrm{~m}, 1 \mathrm{H}), 1.30-1.20(\mathrm{~m}, 1 \mathrm{H}), 1.01-0.95(\mathrm{~m}, 28 \mathrm{H}), 0.84$ $(\mathrm{d}, J=7.1 \mathrm{~Hz}, 3 \mathrm{H}), 0.81(\mathrm{~d}, J=6.5 \mathrm{~Hz}, 3 \mathrm{H}), 0.71(\mathrm{~d}, J=6.9 \mathrm{~Hz}, 3 \mathrm{H})$.

${ }^{13} \mathrm{C}-\mathrm{NMR}\left(75 \mathrm{MHz}_{\mathrm{CDCl}}, \mathrm{ppm}\right): \delta=112.6,80.2,47.5,42.6,34.9,34.6,32.5,28.7,24.2,22.3,21.3,18.6,15.7$, 11.3.

FT-IR $\left(\mathbf{A T R}, \mathbf{c m}^{-1}\right): \tilde{v}=2942,2924,2891,2865,2170,1647,1462,1375,1259,1060,996,908,884,844,735$, 678.

MS (EI, 70 eV): $m / z(\%)=224$ (4), 323 (12), 291 (3), 278 (2), 277 (9), 276 (40), 275 (100), 273 (6)

HR-MS (EI, 70 eV): [ $\left.\mathrm{C}_{21} \mathrm{H}_{38} \mathrm{Si}\right]$, calcd.: 275.2201; found: 275.2192 [M+ $\left.{ }^{+}-\mathrm{Pr}\right]$. 


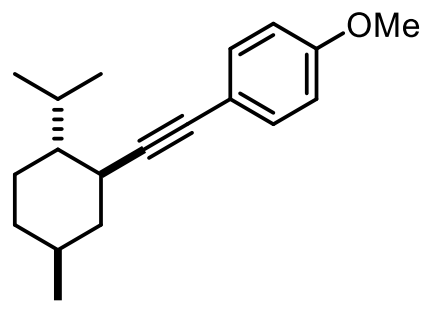

Isolated yield: $100 \mathrm{mg}, 0.37 \mathrm{mmol}, 74 \%$, colorless oil

dr: 99:1.

Purification: $i$-hexane: ethyl acetate $=100: 2$

${ }^{1} \mathrm{H}-\mathrm{NMR}\left(400 \mathrm{MHz}^{\mathrm{CDCl}} \mathrm{CD}_{3} \mathrm{ppm}\right): \delta=7.26(\mathrm{~d}, J=8.9 \mathrm{~Hz}, 2 \mathrm{H}), 6.73(\mathrm{~d}, J=8.6 \mathrm{~Hz}, 2 \mathrm{H}), 3.72(\mathrm{~s}, 3 \mathrm{H}), 2.31-2.21(\mathrm{~m}$, $2 \mathrm{H}), 2.03-1.95(\mathrm{~m}, 1 \mathrm{H}), 1.70-1.62(\mathrm{~m}, 1 \mathrm{H}), 1.60-1.54(\mathrm{~m}, 1 \mathrm{H}), 1.37-1.24(\mathrm{~m}, 1 \mathrm{H}), 1.24-1.04(\mathrm{~m}, 3 \mathrm{H}), 0.99$ $-0.90(\mathrm{~m}, 1 \mathrm{H}), 0.87(\mathrm{~d}, J=7.1 \mathrm{~Hz}, 3 \mathrm{H}), 0.83(\mathrm{~d}, J=6.5 \mathrm{~Hz}, 3 \mathrm{H}), 0.75(\mathrm{~d}, J=6.9 \mathrm{~Hz}, 3 \mathrm{H})$.

${ }^{13} \mathrm{C}-\mathrm{NMR}\left(100 \mathrm{MHz} \mathrm{CDCl}_{3}, \mathrm{ppm}\right): \delta=158.9,132.9,116.4,113.7,91.9,81.1,55.3,47.5,42.5,34.9,34.1,32.5$, $28.9,24.3,22.3,21.4,15.9$.

FT-IR $\left(A T R, \mathbf{c m}^{-1}\right): \tilde{v}=2954,2869,2253,1607,1509,1288,1246,1172,1035,906,831,731$.

MS (EI, 70 eV): m/z (\%) = 270 (15), 255 (100), 213 (14), 147 (35), 145 (9), 120 (20), 114 (2), 42 (4), 40 (8).

HR-MS (EI, $70 \mathrm{eV}$ ): [ $\mathrm{C}_{19} \mathrm{H}_{26} \mathrm{O}$ ], calcd.: 270.1984; found: 238.1356.

tert-Butyl(((1R,2S)-2-((4-methoxyphenyl)ethynyl)cyclopentyl)oxy)dimethylsilane (3c)

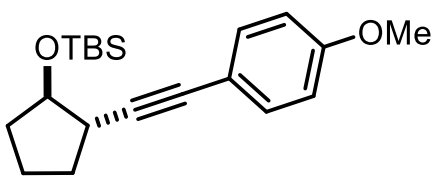

Isolated yield: $114 \mathrm{mg}, 0.34 \mathrm{mmol}, 69 \%$, colorless oil

dr: 99:1.

Purification: $i$-hexane:ethyl acetate $=100: 2$

${ }^{1} \mathrm{H}-\mathrm{NMR}\left(400 \mathrm{MHz} \mathrm{CDCl}_{3}, \mathrm{ppm}\right): \delta=7.24-7.18(\mathrm{~m}, 2 \mathrm{H}), 6.73-6.67(\mathrm{~m}, 2 \mathrm{H}), 4.12(\mathrm{q}, J=5.7 \mathrm{~Hz}, 1 \mathrm{H}), 3.69(\mathrm{~s}$, $3 \mathrm{H}), 2.67-2.57(\mathrm{~m}, 1 \mathrm{H}), 2.05-1.96(\mathrm{~m}, 1 \mathrm{H}), 1.90-1.80(\mathrm{~m}, 1 \mathrm{H}), 1.70-1.55(\mathrm{~m}, 3 \mathrm{H}), 1.52-1.41(\mathrm{~m}, 1 \mathrm{H}), 0.80$ $(\mathrm{s}, 9 \mathrm{H}), 0.02(\mathrm{~s}, 3 \mathrm{H}), 0.01(\mathrm{~s}, 3 \mathrm{H})$. 
${ }^{13} \mathrm{C}-\mathrm{NMR}\left(100 \mathrm{MHz}, \mathrm{CDCl}_{3}, \mathrm{ppm}\right): \delta=158.9,132.8,116.2,113.8,91.1,81.2,79.9,55.3,40.2,34.6,31.0,25.9$, $21.9,18.2,-4.5,-4.7$.

FT-IR (ATR, cm $\left.^{-1}\right): \tilde{v}=2955,2856,2359,1607,1509,1287,1247,1090,1038,906,832,776,731$.

MS (El, $70 \mathrm{eV}): m / z(\%)=273$ (29), 200 (13), 199 (100), 171 (5), 164 (4), 121 (4), 75 (15), 73 (15).

HR-MS (EI, $70 \mathrm{eV})$ : [ $\left.\mathrm{C}_{16} \mathrm{H}_{21} \mathrm{O}_{2} \mathrm{Si}\right]$, calcd.: 273.1311; found: 273.1326 [M+ $\left.\mathrm{M}^{+}-t \mathrm{Bu}\right]$.

tert-Butyl(((1R,2S,5R)-2-isopropyl-5-methylcyclohexyl)ethynyl)dimethylsilane (3d)

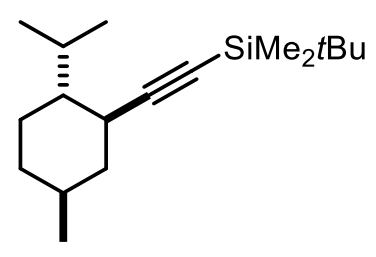

Isolated yield: $79 \mathrm{mg}, 0.28 \mathrm{mmol}, 61 \%$, colorless oil

dr: 99:1.

Purification: $i$-hexane

${ }^{1} \mathrm{H}-\mathrm{NMR}\left(300 \mathrm{MHz}, \mathrm{CDCl}_{3}, \mathrm{ppm}\right): \delta=2.29(\mathrm{pd}, J=7.0,2.9 \mathrm{~Hz}, 1 \mathrm{H}), 2.21-2.13(\mathrm{~m}, 1 \mathrm{H}), 1.98(\mathrm{dtd}, J=13.0,3.5$, $2.2 \mathrm{~Hz}, 1 \mathrm{H}), 1.73-1.67(\mathrm{~m}, 1 \mathrm{H}), 1.64-1.57(\mathrm{~m}, 1 \mathrm{H}), 1.38-1.25(\mathrm{~m}, 1 \mathrm{H}), 1.23-1.03(\mathrm{~m}, 2 \mathrm{H}), 0.97-0.90(\mathrm{~m}$, $14 \mathrm{H}), 0.88(\mathrm{~d}, J=6.5 \mathrm{~Hz}, 3 \mathrm{H}), 0.77(\mathrm{~d}, \mathrm{~J}=6.9 \mathrm{~Hz}, 3 \mathrm{H}), 0.07(\mathrm{~s}, 6 \mathrm{H})$.

${ }^{13} \mathrm{C}-\mathrm{NMR}\left(75 \mathrm{MHz}, \mathrm{CDCl}_{3}, \mathrm{ppm}\right): \delta=111.5,82.9,47.5,42.6,35.0,34.7,32.6,28.8,26.7,26.3,26.2,24.4,22.4$, $21.4,16.7,15.9,-4.2,-4.2$.

FT-IR (ATR, $\left.\mathbf{c m}^{-1}\right): \tilde{v}=2955,2929,2857,2167,1471,1249,908,908,837,824,810,775,735,686$.

MS (EI, 70 eV): $m / z(\%)=221(5), 74(8), 73(100), 57(11)$.

HR-MS (EI, $70 \mathrm{eV}$ ): [ $\left.\mathrm{C}_{18} \mathrm{H}_{34} \mathrm{Si}\right]$, calcd.: 221.1720; found: $221.1720\left[\mathrm{M}^{+}-\mathrm{C}_{4} \mathrm{H}_{9}\right]$.

(((1S,2R,5S)-2-Isopropyl-5-methylcyclohexyl)ethynyl)trimethylsilane (3e)

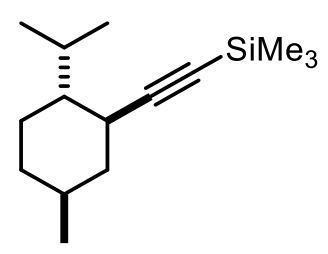

Isolated yield: $92 \mathrm{mg}, 0.39 \mathrm{mmol}, 78 \%$, colorless oil 
dr: 99:1.

Purification: $i$-hexane

${ }^{1} \mathrm{H}-\mathrm{NMR}\left(400 \mathrm{MHz}, \mathrm{CDCl}_{3}, \mathrm{ppm}\right): \delta=2.25(\mathrm{pd}, J=7.1,2.8 \mathrm{~Hz}, 1 \mathrm{H}), 2.17(\mathrm{td}, J=11.5,3.5 \mathrm{~Hz}, 1 \mathrm{H}), 1.98(\mathrm{dd}, J=$ 12.8, 3.2 Hz, 1H), $1.75-1.66(\mathrm{~m}, 1 \mathrm{H}), 1.64-1.57(\mathrm{~m}, 1 \mathrm{H}), 1.39-1.24(\mathrm{~m}, 1 \mathrm{H}), 1.24-1.10(\mathrm{~m}, 2 \mathrm{H}), 1.01-0.94$ $(\mathrm{m}, 2 \mathrm{H}), 0.93(\mathrm{~d}, J=6.8 \mathrm{~Hz}, 3 \mathrm{H}), 0.88(\mathrm{~d}, J=6.5 \mathrm{~Hz}, 3 \mathrm{H}), 0.78(\mathrm{~d}, J=6.9 \mathrm{~Hz}, 3 \mathrm{H}), 0.14(\mathrm{~s}, 9 \mathrm{H})$.

${ }^{13} \mathrm{C}-\mathrm{NMR}\left(100 \mathrm{MHz}, \mathrm{CDCl}_{3}, \mathrm{ppm}\right): \delta=110.6,84.4,46.8,42.0,34.5,34.1,32.1,28.3,24.0,22.0,21.0,15.5,0.0$.

FT-IR $\left(A T R\right.$, cm $\left.^{-1}\right): \widetilde{v}=2956,1704,1456,1407,1386,1368,1248,1131,930,835,758,696,668$.

MS (EI, 70 eV): m/z (\%) = 237 (4), $236(21), 222$ (25), 221 (100), 163 (26), 162 (66), 147 (32), 140 (15), 109 (13), $73(55)$.

HR-MS (EI, $70 \mathrm{eV}):\left[\mathrm{C}_{15} \mathrm{H}_{28} \mathrm{Si}\right]$, calcd.: 236.1960; found: 236.1945.

tert-Butyldimethyl(((1R,2S,5R)-5-methyl-2-(prop-1-en-2-yl)cyclohexyl)ethynyl)silane (3f)

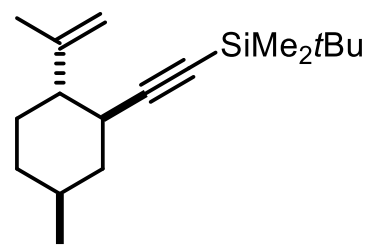

Isolated yield: $76 \mathrm{mg}, 0.27 \mathrm{mmol}, 55 \%$, colorless oil

dr: 99:1.

Purification: $i$-hexane

${ }^{1} \mathrm{H}-\mathrm{NMR}\left(300 \mathrm{MHz}, \mathrm{CDCl}_{3}, \mathrm{ppm}\right): \delta=4.75(\mathrm{q}, J=1.2 \mathrm{~Hz}, 2 \mathrm{H}), 2.29$ (ddd, $\left.J=11.9,11.0,3.5 \mathrm{~Hz}, 1 \mathrm{H}\right), 2.01$ (dtd, $J=$ 13.1, 3.5, $2.0 \mathrm{~Hz}, 1 \mathrm{H}), 1.93(\mathrm{ddd}, J=12.0,11.0,3.3 \mathrm{~Hz}, 1 \mathrm{H}), 1.70(\mathrm{~m}, 3 \mathrm{H}), 1.69-1.62(\mathrm{~m}, 2 \mathrm{H}), 1.42-1.23(\mathrm{~m}$, $2 \mathrm{H}), 1.17-1.06(\mathrm{~m}, 1 \mathrm{H}), 1.06-0.93(\mathrm{~m}, 1 \mathrm{H}), 0.90(\mathrm{~d}, J=5.7 \mathrm{~Hz}, 12 \mathrm{H}), 0.04(\mathrm{~s}, 6 \mathrm{H})$.

${ }^{13} \mathrm{C}-\mathrm{NMR}\left(75 \mathrm{MHz}, \mathrm{CDCl}_{3}, \mathrm{ppm}\right): \delta=148.1,111.2,110.9,82.7,51.3,41.8,34.8,34.7,32.3,32.0,26.2,22.4,19.8$, $16.7,1.2,-4.2,-4.3$.

FT-IR $\left(A T R\right.$, cm $\left.^{-1}\right): \tilde{v}=2952,2927,2865,2171,1647,1456,1248,1006,907,845,825,811,774,734,685,650$.

MS (EI, $70 \mathrm{eV}): m / z$ (\%) = 276 (2), 221 (16), 220 (69), 219 (100), 217 (13), 178 (11), 177 (65), 163 (9), 160 (18), 159 (37), 157 (14), 151 (9), 149 (17), 135 (14), 123 (16), 119 (11), 109 (38), 105 (11), 98 (14), 96 (13), 83 (22), 81 (11), 73 (98), 59 (80), 57 (21), 55 (15), 43 (9), 43 (11), 41 (24).

HR-MS (EI, $70 \mathrm{eV}):\left[\mathrm{C}_{18} \mathrm{H}_{32} \mathrm{Si}\right]$, calcd.: 276.2273; found: 276.2272. 
Triisopropyl(((1S,2S,5S)-5-methyl-2-(prop-1-en-2-yl)cyclohexyl)ethynyl)silane (3g)

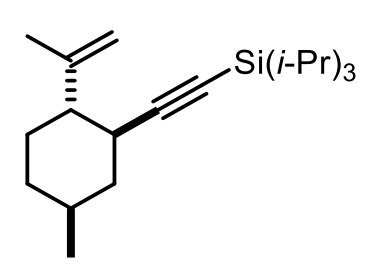

Isolated yield: $108 \mathrm{mg}, 0.34 \mathrm{mmol}, 68 \%$, colorless oil

${ }^{1} \mathrm{H}-\mathrm{NMR}\left(300 \mathrm{MHz} \mathrm{CDCl}_{3}, \mathrm{ppm}\right): \delta=4.74(\mathrm{dt}, J=5.4,1.8 \mathrm{~Hz}, 2 \mathrm{H}), 2.36-2.22(\mathrm{~m}, 1 \mathrm{H}), 2.02(\mathrm{dtd}, J=13.1,3.5$, $1.9 \mathrm{~Hz}, 1 \mathrm{H}), 1.94(\mathrm{td}, J=11.8,3.3 \mathrm{~Hz}, 1 \mathrm{H}), 1.71(\mathrm{t}, J=1.1 \mathrm{~Hz}, 3 \mathrm{H}), 1.69-1.62(\mathrm{~m}, 1 \mathrm{H}), 1.44-1.22(\mathrm{~m}, 2 \mathrm{H}), 1.21-$ $0.92(\mathrm{~m}, 23 \mathrm{H}), 0.90(\mathrm{~d}, J=6.5 \mathrm{~Hz}, 3 \mathrm{H})$.

${ }^{13}$ C-NMR (75 MHz, CDCl 3 , ppm): $\delta=148.1,112.0,111.2,80.0,78.7,51.5,42.0,34.8,34.7,32.3,32.1,22.4,19.6$, $18.8,18.8,18.8,11.4,11.4$.

FT-IR $\left(\mathbf{A T R}, \mathbf{c m}^{-1}\right): \tilde{v}=2942,2924,2891,2865,2170,1647,1462,1375,1259,1060,996,908,884,844,735$, 678.

MS (EI, 70 eV): m/z (\%) = 323 (23), 307 (3), 277 (10), 276 (40), 275 (100), 273 (6).

HR-MS (EI, $70 \mathrm{eV}$ ): [ $\left.\mathrm{C}_{21} \mathrm{H}_{38} \mathrm{Si}\right]$, calcd.: 275.2195; found: $275.2192\left[\mathrm{M}^{+}-\mathrm{C}_{3} \mathrm{H}_{7}\right]$.

tert-butyl(((1R,2S)-2-(cyclopentylethynyl)cyclopentyl)oxy)dimethylsilane (3h)

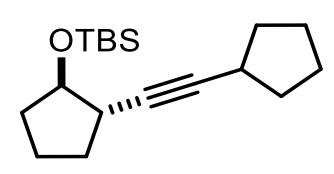

Isolated yield: $85 \mathrm{mg}, 0.29 \mathrm{mmol}, 58 \%$, colorless oil

dr: 99:1.

Purification: $i$-hexane: ethyl acetate $=99: 1$

${ }^{1} \mathrm{H}-\mathrm{NMR}\left(400 \mathrm{MHz} \mathrm{CDCl}_{3}, \mathrm{ppm}\right): \delta=3.99(\mathrm{q}, J=5.7 \mathrm{~Hz}, 1 \mathrm{H}), 2.49(\mathrm{pd}, J=7.5,2.1 \mathrm{~Hz}, 1 \mathrm{H}), 2.41$ (tdd, $J=7.7,5.5$, $2.1 \mathrm{~Hz}, 1 \mathrm{H}), 1.98-1.87(\mathrm{~m}, 1 \mathrm{H}), 1.87-1.75(\mathrm{~m}, 3 \mathrm{H}), 1.67-1.54(\mathrm{~m}, 4 \mathrm{H}), 1.51-1.37(\mathrm{~m}, 6 \mathrm{H}), 0.81(\mathrm{~s}, 9 \mathrm{H}), 0.01$ $(\mathrm{s}, 3 \mathrm{H}), 0.00(\mathrm{~s}, 3 \mathrm{H})$.

${ }^{13} \mathrm{C}-\mathrm{NMR}\left(100 \mathrm{MHz}, \mathrm{CDCl}_{3}, \mathrm{ppm}\right): \delta=85.4,82.2,80.1,39.6,34.4,34.1,31.3,30.4,25.9,24.9,21.8$, $18.2,-4.6,-4.7$. 
FT-IR (ATR, cm ${ }^{-1}$ ): $\tilde{v}=2954,2931,2858,1710,1472,1361,1251,1085,1005,939,895,865,835,775,668$.

MS (EI, 70 eV): m/z (\%) = 291 (1), 235 (28), 161 (24), 159 (63), 91 (32), 75 (100), 73 (31), 67 (12).

HR-MS (EI, $70 \mathrm{eV}$ ): [ $\left.\mathrm{C}_{18} \mathrm{H}_{32} \mathrm{OSi}\right]$, calcd.: 291.2148; found: 291.2144.

tert-butyl(((1R,2S)-2-(cyclohex-1-en-1-ylethynyl)cyclopentyl)oxy)dimethylsilane (3i)

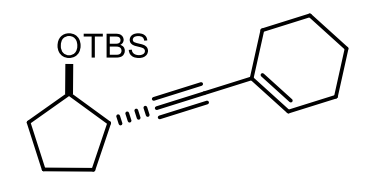

Isolated yield: $104 \mathrm{mg}, 0.34 \mathrm{mmol}, 68 \%$, colorless oil

dr: 99:1.

Purification: $i$-hexane:ethyl acetate $=99: 1$

${ }^{1} \mathrm{H}-\mathrm{NMR}\left(400 \mathrm{MHz}^{\mathrm{CDCl}} \mathrm{CDpm}_{3}\right): \delta=5.99(\mathrm{dt}, J=4.0,2.1 \mathrm{~Hz}, 1 \mathrm{H}), 4.13(\mathrm{q}, J=5.4 \mathrm{~Hz}, 1 \mathrm{H}), 2.67-2.57(\mathrm{~m}, 1 \mathrm{H})$, $2.11-2.03(\mathrm{~m}, 5 \mathrm{H}), 1.91(\mathrm{ddt}, J=12.6,8.6,6.3 \mathrm{~Hz}, 1 \mathrm{H}), 1.72-1.51(\mathrm{~m}, 8 \mathrm{H}), 0.89(\mathrm{~s}, 9 \mathrm{H}), 0.09(\mathrm{~s}, 3 \mathrm{H}), 0.08(\mathrm{~s}$, $3 \mathrm{H})$.

${ }^{13} \mathrm{C}-\mathrm{NMR}\left(100 \mathrm{MHz}, \mathrm{CDCl}_{3}, \mathrm{ppm}\right): \delta=133.2,121.0,89.7,83.2,79.9,40.1,34.51,31.2,29.6,25.9,25.6,22.4$, $21.9,21.6,18.2,-4.7,-4.8$.

FT-IR (ATR, $\mathbf{c m}^{-1}$ ): $\tilde{v}=2950,2930,2857,1715,1674,1472,1462,1447,1388,1360,1250,1170,1087,1005$, $939,867,834,775,668$.

MS (El, 70 eV): m/z (\%) = 304 (1), 247 (47), 174 (15), 173 (100), 171 (53), 129 (15), 91 (15), 75 (68), 73 (34).

HR-MS (EI, $70 \mathrm{eV}$ ): [ $\left.\mathrm{C}_{19} \mathrm{H}_{32} \mathrm{OSi}\right]$, calcd.: 304.2222; found: 304.2202.

4-(((1S,2R)-2-((tert-butyldimethylsilyl)oxy)cyclohexyl)ethynyl)benzonitrile (3j)<smiles>N#Cc1ccc(C#C[C@H]2CCCCC2[Se-](F)(F)F)cc1</smiles>

Isolated yield: $105 \mathrm{mg}, 0.31 \mathrm{mmol}, 62 \%$, yellow oil

dr: 99:1. 
Purification: $i$-hexane:ethyl acetate $=9: 1$

${ }^{1} \mathrm{H}-\mathrm{NMR}\left(400 \mathrm{MHz}^{\mathrm{CDCl}}{ }_{3}, \mathrm{ppm}\right): \delta=7.55(\mathrm{~d}, J=8.3 \mathrm{~Hz}, 2 \mathrm{H}), 7.44(\mathrm{~d}, J=8.3 \mathrm{~Hz}, 2 \mathrm{H}), 3.69-3.58(\mathrm{~m}, 1 \mathrm{H}), 2.52$ (ddd, $J=10.0,8.1,3.9 \mathrm{~Hz}, 1 \mathrm{H}), 2.09-1.99(\mathrm{~m}, 1 \mathrm{H}), 1.95-1.86(\mathrm{~m}, 1 \mathrm{H}), 1.78-1.61(\mathrm{~m}, 2 \mathrm{H}), 1.53-1.40(\mathrm{~m}, 1 \mathrm{H})$, $1.37-1.19(\mathrm{~m}, 3 \mathrm{H}), 0.88(\mathrm{~s}, 9 \mathrm{H}), 0.07(\mathrm{~s}, 6 \mathrm{H})$.

${ }^{13} \mathrm{C}-\mathrm{NMR}\left(100 \mathrm{MHz} \mathrm{CDCl}_{3}, \mathrm{ppm}\right): \delta=132.1,131.9,129.2,118.7,110.8,98.3,80.4,73.1,38.7,34.5,30.3,29.7$, $25.8,24.3,23.5,18.1,-4.4,-4.6$.

FT-IR $\left(\mathbf{A T R}, \mathbf{c m}^{-1}\right): \tilde{v}=2929,2856,2227,1605,1501,1472,1462,1249,1131,1100,1059,1039,1022,1006$, $938,876,833,773,668$.

MS (EI, 70 eV): m/z (\%) = 339 (1), 284 (14), 283 (43), 282 (22), 208 (17), 184 (25), 15 (13), 75 (100), 44.

HR-MS (EI, $70 \mathrm{eV}$ ): [ $\left.\mathrm{C}_{21} \mathrm{H}_{29} \mathrm{NOSi}\right]$, calcd.: 339.2018; found: 339.1996.

tert-Butyldimethyl((trans-2-((triisopropylsilyl)ethynyl)cyclohexyl)oxy)silane (3k)<smiles>CC(C)[SiH2]C#C[C@H]1CCCCC1[OH+]</smiles>

Isolated yield: $910 \mathrm{mg}, 77 \%$, colorless oil

dr: 99:1.

Purification: $i$-hexane: $\mathrm{EE}=100: 2$

${ }^{1} \mathrm{H}-\mathrm{NMR}\left(300 \mathrm{MHz}, \mathrm{CDCl}_{3}, \mathrm{ppm}\right): \delta=3.71(\mathrm{td}, J=6.4,3.1 \mathrm{~Hz}, 1 \mathrm{H}), 2.44(\mathrm{td}, J=6.8,4.1 \mathrm{~Hz}, 1 \mathrm{H}), 2.03-1.85(\mathrm{~m}$, $1 \mathrm{H}), 1.73-1.58(\mathrm{~m}, 2 \mathrm{H}), 1.52-1.41(\mathrm{~m}, 1 \mathrm{H}), 1.32$ (qt, $J=6.2,3.4 \mathrm{~Hz}, 3 \mathrm{H}), 1.15-0.96(\mathrm{~m}, 21 \mathrm{H}), 0.89(\mathrm{~s}, 10 \mathrm{H})$, $0.08(\mathrm{~d}, J=5.6 \mathrm{~Hz}, 6 \mathrm{H})$.

${ }^{13} \mathrm{C}-\mathrm{NMR}\left(75 \mathrm{MHz}^{\mathrm{CDCl}}{ }_{3}, \mathrm{ppm}\right): \delta=111.7,81.7,72.0,47.8,37.9,32.7,29.3,26.1,26.0,23.4,22.3,18.8,18.2$, $11.5,-4.4,-4.5$.

FT-IR $\left(\mathbf{A T R}, \mathbf{c m}^{-1}\right): \tilde{v}=2937,2892,2863,2359,2166,1463,1366,1383,1252,1157,1105,1068,1022,942,908$, $875,836,775,735,676$.

MS (EI, 70 eV): m/z (\%) = 394 (1), 393 (1), 337 (8), 309 (18), 296 (26), 295 (100), 267 (19), 253 (4), 225 (8).

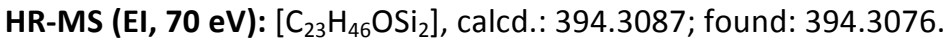


1-((1R,2S,5R)-2-Isopropyl-5-methylcyclohexyl)-4-(trifluoromethyl)benzene (4a)

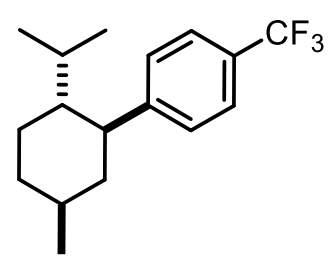

Isolated yield: $201 \mathrm{mg}, 0.36 \mathrm{mmol}, 71 \%$, colorless oil

dr: 99:1.

Purification: $i$-hexane:ethyl acetate $=100: 3$

${ }^{1} \mathrm{H}-\mathrm{NMR}\left(400 \mathrm{MHz}_{\mathrm{CDCl}}, \mathrm{ppm}\right): \delta=7.55(\mathrm{~d}, J=8.5 \mathrm{~Hz}, 2 \mathrm{H}), 7.33-7.16(\mathrm{~m}, 2 \mathrm{H}), 2.49(\mathrm{td}, J=11.6,3.5 \mathrm{~Hz}, 1 \mathrm{H})$, $1.84-1.66(\mathrm{~m}, 2 \mathrm{H}), 1.56(\mathrm{~s}, 1 \mathrm{H}), 1.53-1.41(\mathrm{~m}, 2 \mathrm{H}), 1.36-0.95(\mathrm{~m}, 4 \mathrm{H}), 0.89(\mathrm{~d}, J=6.5 \mathrm{~Hz}, 3 \mathrm{H}), 0.79(\mathrm{~d}, J=6.9$ $\mathrm{Hz}, 3 \mathrm{H}), 0.65(\mathrm{~d}, J=6.9 \mathrm{~Hz}, 3 \mathrm{H})$.

${ }^{13} \mathrm{C}-\mathrm{NMR}\left(100 \mathrm{MHz}_{\mathrm{CDCl}}, \mathrm{ppm}\right): \delta=152.4,132.2,128.3,119.1,109.5,48.3,47.2,44.8,35.0,33.1,27.6,24.36$, $22.4,21.4,15.3$.

FT-IR (ATR, $\left.\mathbf{c m}^{-1}\right): \tilde{v}=2957,2871,1447,1324,1164,1125,1073,906,799,732,703,665$.

MS (EI, 70 eV): m/z (\%) = 284 (56), 199 (74), 173 (51), 172 (94), 159 (100), 112 (42), 69 (53).

HR-MS (EI, $70 \mathrm{eV}):$ [ $\left.\mathrm{C}_{17} \mathrm{H}_{23} \mathrm{~F}_{3}\right]$, calcd.: 284.1752; found: 284.1735.

1-(4-((1R,2S,5R)-2-Isopropyl-5-methylcyclohexyl)phenyl)-2,2-dimethylpropan-1-one (4b)

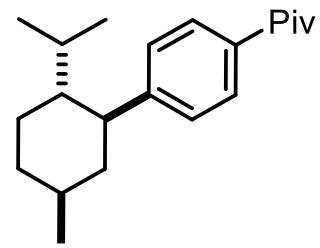

Isolated yield: $221 \mathrm{mg}, 0.35 \mathrm{mmol}, 70 \%$, colorless oil

dr: 99:1.

Purification: $i$-hexane:ethyl acetate $=95: 5$

${ }^{1} \mathrm{H}-\mathrm{NMR}\left(400 \mathrm{MHz} \mathrm{CDCl}_{3}, \mathrm{ppm}\right): \delta=7.90(\mathrm{~d}, J=8.3 \mathrm{~Hz}, 2 \mathrm{H}), 7.19(\mathrm{~d}, J=8.2 \mathrm{~Hz}, 2 \mathrm{H}), 2.48(\mathrm{td}, J=11.6,3.5 \mathrm{~Hz}$, $1 \mathrm{H}), 1.86-1.68(\mathrm{~m}, 3 \mathrm{H}), 1.57(\mathrm{~s}, 9 \mathrm{H}), 1.52-1.32(\mathrm{~m}, 3 \mathrm{H}), 1.19-0.97(\mathrm{~m}, 3 \mathrm{H}), 0.88(\mathrm{~d}, J=6.5 \mathrm{~Hz}, 3 \mathrm{H}), 0.77(\mathrm{~d}, J=$ $7.0 \mathrm{~Hz}, 3 \mathrm{H}), 0.65(\mathrm{~d}, J=6.9 \mathrm{~Hz}, 3 \mathrm{H})$. 
${ }^{13} \mathrm{C}-\mathrm{NMR}\left(100 \mathrm{MHz}, \mathrm{CDCl}_{3}, \mathrm{ppm}\right): \delta=165.8,151.7,129.5,80.5,48.1,47.3,44.9,35.2,33.1,28.2,27.5,24.5$, $22.4,21.4,15.3$.

FT-IR (ATR, cm $\left.^{-1}\right): \tilde{v}=2954,2918,1711,1608,1455,1367,1290,1255,1165,1116,1102,908,850,773,733$, 708.

MS (EI, $70 \mathrm{eV}): m / z(\%)=261$ (33), 250 (100), 243 (56), 149 (43), 148 (73), 135 (35), 125 (26), 91 (23), 69 (37), 57 (87), 40 (32).

HR-MS (EI, $70 \mathrm{eV}):\left[\mathrm{C}_{21} \mathrm{H}_{32} \mathrm{O}_{2}\right]$, calcd.: 316.2402; found: 316.2387.

4-((1R,2S,5R)-2-Isopropyl-5-methylcyclohexyl)benzonitrile (4c)

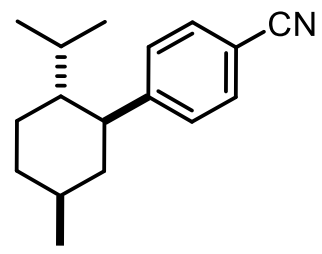

Isolated yield: $147 \mathrm{mg}, 0.31 \mathrm{mmol}, 61 \%$, colorless oil

dr: 99:1.

Purification: $i$-hexane:ethyl acetate $=100: 3$

${ }^{1} \mathrm{H}-\mathrm{NMR}\left(400 \mathrm{MHz}, \mathrm{CDCl}_{3}, \mathrm{ppm}\right): \delta=7.55(\mathrm{~d}, J=8.5 \mathrm{~Hz}, 2 \mathrm{H}), 7.33-7.16(\mathrm{~m}, 2 \mathrm{H}), 2.49(\mathrm{td}, J=11.6,3.5 \mathrm{~Hz}, 1 \mathrm{H})$, $1.84-1.66(\mathrm{~m}, 2 \mathrm{H}), 1.56(\mathrm{~s}, 1 \mathrm{H}), 1.53-1.41(\mathrm{~m}, 2 \mathrm{H}), 1.36-0.95(\mathrm{~m}, 5 \mathrm{H}), 0.89(\mathrm{~d}, J=6.5 \mathrm{~Hz}, 3 \mathrm{H}), 0.79(\mathrm{~d}, J=6.9$ $\mathrm{Hz}, 3 \mathrm{H}), 0.65(\mathrm{~d}, J=6.9 \mathrm{~Hz}, 3 \mathrm{H})$.

${ }^{13} \mathrm{C}-\mathrm{NMR}\left(100 \mathrm{MHz}, \mathrm{CDCl}_{3}, \mathrm{ppm}\right): \delta=152.4,132.2,128.3,119.1,109.5,48.3,47.2,44.8,35.0,33.1,27.6,24.36$, $22.4,21.4,15.3$.

FT-IR $\left(A T R\right.$, cm $\left.^{-1}\right): \tilde{v}=1413,1342,1322,1253,1165,1125,1097,1076,1045,895,796,702,665$.

MS (EI, $70 \mathrm{eV}): m / z(\%)=241$ (100), 156 (73), 142 (53), 130 (95), 116 (52), 81 (53).

HR-MS (EI, $70 \mathrm{eV}):\left[\mathrm{C}_{17} \mathrm{H}_{23} \mathrm{~N}\right]$, calcd.: 241.1830; found: 241.1821.

4-((1S,2R,5S)-2-Isopropyl-5-methylcyclohexyl)phenyl pivalate (4d) 


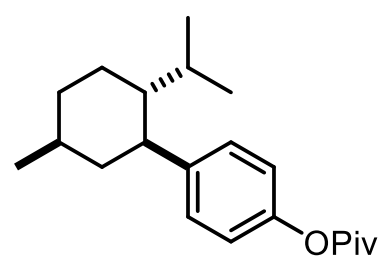

Isolated yield: $119 \mathrm{mg}, 0.38 \mathrm{mmol}, 75 \%$, colorless crystals

dr: 99:1.

Purification: $i$-hexane:ethyl acetate $=96: 4$

${ }^{1} \mathrm{H}-\mathrm{NMR}\left(400 \mathrm{MHz}, \mathrm{CDCl}_{3}, \mathrm{ppm}\right): \delta=7.15(\mathrm{~d}, J=8.0 \mathrm{~Hz}, 2 \mathrm{H}), 6.97(\mathrm{~d}, J=8.0 \mathrm{~Hz}, 2 \mathrm{H}), 2.43(\mathrm{td}, J=11.7,3.4 \mathrm{~Hz}$, $1 \mathrm{H}), 1.88-1.67(\mathrm{~m}, 3 \mathrm{H}), 1.52-1.37(\mathrm{~m}, 3 \mathrm{H}), 1.35(\mathrm{~s}, 9 \mathrm{H}), 1.20-0.94(\mathrm{~m}, 3 \mathrm{H}), 0.89(\mathrm{~d}, J=6.4 \mathrm{~Hz}, 3 \mathrm{H}), 0.78(\mathrm{~d}, J$ $=6.8 \mathrm{~Hz}, 3 \mathrm{H}), 0.65(\mathrm{~d}, J=6.6 \mathrm{~Hz}, 3 \mathrm{H})$.

${ }^{13} \mathrm{C}-\mathrm{NMR}\left(100 \mathrm{MHz} \mathrm{CDCl}_{3}, \mathrm{ppm}\right): \delta=177.3,148.9,143.9,128.2,121.2,47.6,47.4,45.3,39.1,35.3,33.2,27.3$, $27.2,27.1,24.5,22.6,22.5,21.5,15.3$.

FT-IR (ATR, $\left.\mathrm{cm}^{-1}\right): \tilde{v}=2963,2921,2872,2842,1744,1730,1504,1492,1480,1462,1454,1442,1398,1384$, $1366,1277,1231,1202,1194,1165,1118,1101,1068,1053,1030,1014,968,944,937,919,896,872,852$, $832,819,808,796,784,759,723,621,604,594,585,574,566,558$.

MS (EI, 70 eV): m/z (\%) = 317 (5), 316 (23), 233 (18), 232 (100), 146 (77), 107 (31), 91 (7), 95 (8), 57 (42), 41 (11).

HR-MS (EI, $70 \mathrm{eV}):\left[\mathrm{C}_{21} \mathrm{H}_{32} \mathrm{O}_{2}\right]$, calcd.: 316.2402; found: 316.2388.

4-((1S,2R,5S)-2-Isopropyl-5-methylcyclohexyl)-N,N-dimethylaniline (4e)

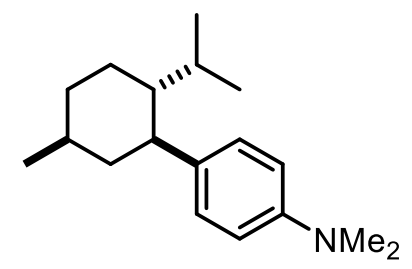

Isolated yield: $80 \mathrm{mg}, 0.31 \mathrm{mmol}, 62 \%$, white solid

dr: 91:9.

Purification: $i$-hexane:ethyl acetate $=98: 2$

${ }^{1} \mathrm{H}-\mathrm{NMR}\left(400 \mathrm{MHz}, \mathrm{CDCl}_{3}, \mathrm{ppm}\right): \delta=7.04(\mathrm{~d}, J=8.1 \mathrm{~Hz}, 2 \mathrm{H}), 6.70(\mathrm{~d}, J=8.1 \mathrm{~Hz}, 2 \mathrm{H}), 2.92(\mathrm{~s}, 6 \mathrm{H}), 2.33(\mathrm{td}, J=$ 11.6, 3.4 Hz, 1H), $1.87-1.65(\mathrm{~m}, 3 \mathrm{H}), 1.54-1.28(\mathrm{~m}, 3 \mathrm{H}), 1.20-0.94(\mathrm{~m}, 3 \mathrm{H}), 0.88(\mathrm{~d}, J=6.5 \mathrm{~Hz}, 3 \mathrm{H}), 0.79(\mathrm{dd}$, $J=6.7 \mathrm{~Hz}, 1.2,3 \mathrm{H}), 0.67(\mathrm{dd}, J=7.0,1.2 \mathrm{~Hz}, 3 \mathrm{H})$. 
${ }^{13} \mathrm{C}-\mathrm{NMR}\left(100 \mathrm{MHz}, \mathrm{CDCl}_{3}, \mathrm{ppm}\right): \delta=148.8,134.9,128.0,112.8,47.6,46.9,45.7,40.9,35.4,33.3,27.3,24.6$, 22.6, 21.6, 15.4 .

FT-IR (ATR, $\left.\mathrm{cm}^{-1}\right)$ : $\tilde{v}=2951,2913,2868,2844,2798,1613,1565,1519,1493,1480,1461,1452,1444,1383$, $1366,1344,1277,1206,1191,1162,1138,1097,1082,1059,1038,999,978,948,935,868,818,808,749,730$, $724,690,642,626,595$.

MS (EI, $70 \mathrm{eV}): m / z(\%)=260$ (14), 259 (72), 258 (3), 244 (6), 175 (13), 174 (100), 160 (6), 159 (5), 147 (9), 146 (10), $135(6), 134$ (52).

HR-MS (EI, $70 \mathrm{eV}$ ): [ $\left.\mathrm{C}_{18} \mathrm{H}_{29} \mathrm{~N}\right]$, calcd.: 259.2300; found: 259.2297.

$N, N$-Diethyl-O-(4-((1R,2S,5R)-2-isopropyl-5-methylcyclohexyl)benzoyl)hydroxylamine (4f)

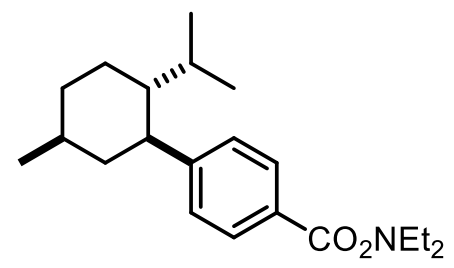

Isolated yield: $248 \mathrm{mg}, 0.38 \mathrm{mmol}, 75 \%$, colorless oil

dr: 99:1.

Purification: $i$-hexane:ethyl acetate $=100: 3$

${ }^{1} \mathrm{H}-\mathrm{NMR}\left(400 \mathrm{MHz}, \mathrm{CDCl}_{3}, \mathrm{ppm}\right): \delta=7.27-7.22(\mathrm{~m}, 1 \mathrm{H}), 7.00-6.91(\mathrm{~m}, 3 \mathrm{H}), 3.49-3.37(\mathrm{~m}, 4 \mathrm{H}), 2.44(\mathrm{td}, \mathrm{J}=$ 11.6, 3.4 Hz, 1H), $1.84-1.70(\mathrm{~m}, 3 \mathrm{H}), 1.55-1.43(\mathrm{~m}, 3 \mathrm{H}), 1.30-1.10(\mathrm{~m}, 8 \mathrm{H}), 1.01(\mathrm{tdd}, J=12.9,11.5,3.4 \mathrm{~Hz}$, $1 \mathrm{H}), 0.89(\mathrm{~d}, J=6.7 \mathrm{~Hz}, 3 \mathrm{H}), 0.81(\mathrm{~d}, J=7.1 \mathrm{~Hz}, 3 \mathrm{H}), 0.69(\mathrm{~d}, J=6.9 \mathrm{~Hz}, 3 \mathrm{H})$.

${ }^{13} \mathrm{C}-\mathrm{NMR}\left(100 \mathrm{MHz}, \mathrm{CDCl}_{3}, \mathrm{ppm}\right): \delta=154.2,151.6,148.1,128.9,118.9,47.9,47.3,45.2,42.1,41.8,35.3,33.2$, $27.4,24.5,22.5,21.5,15.4,14.3,13.4$.

FT-IR (ATR, cm $\left.^{-1}\right): \tilde{v}=2954,2916,2871,1715,1608,1472,1456,1415,1272,1255,1216,1153,970,907,773$, $730,698$.

MS (EI, 70 eV): m/z (\%) = 331 (39), 107 (11), 106 (12), 100 (34), 71 (100), 55 (11), 43 (20), 40 (13).

HR-MS (EI, $70 \mathrm{eV}):\left[\mathrm{C}_{21} \mathrm{H}_{33} \mathrm{NO}_{2}\right]$, calcd.: 331.2511; found: 331.2500 . 
Pentafluoro(3-((1S,2R,5S)-2-isopropyl-5-methylcyclohexyl)phenyl)- $\lambda^{6}$-sulfane (4g)

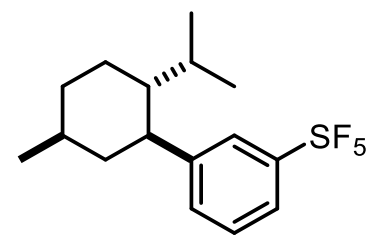

Isolated yield: $116 \mathrm{mg}, 0.34 \mathrm{mmol}, 68 \%$, colorless oil

dr: 99:1.

Purification: $i$-hexane

${ }^{1} \mathrm{H}-\mathrm{NMR}\left(400 \mathrm{MHz}^{\mathrm{CDCl}} 3, \mathrm{ppm}\right): \delta=7.55(\mathrm{~d}, J=8.1 \mathrm{~Hz}, 1 \mathrm{H}), 7.53(\mathrm{~s}, 1 \mathrm{H}), 7.36(\mathrm{t}, J=7.8 \mathrm{~Hz}, 1 \mathrm{H}), 7.30(\mathrm{~d}, J=$ $7.7 \mathrm{~Hz}, 1 \mathrm{H}), 2.51(\mathrm{td}, J=11.7 \mathrm{~Hz}, 3.4 \mathrm{~Hz}, 1 \mathrm{H}), 1.88-1.71(\mathrm{~m}, 3 \mathrm{H}), 1.56-1.28(\mathrm{~m}, 3 \mathrm{H}), 1.25-0.97(\mathrm{~m}, 3 \mathrm{H}), 0.90$ $(\mathrm{d}, J=6.7 \mathrm{~Hz}, 3 \mathrm{H}), 0.81(\mathrm{~d}, J=6.9 \mathrm{~Hz}, 3 \mathrm{H}), 0.67(\mathrm{~d}, J=6.9 \mathrm{~Hz}, 3 \mathrm{H})$.

${ }^{13}$ C-NMR (100 MHz, CDCl 3 , ppm): $\delta=154.1,147.9,130.6,128.6,125.0$ (m), 123.4 (p, J= 4.6 Hz), 48.1, 47.3, $45.1,35.1,33.2,27.5,24.5,22.4,21.4,15.3$.

FT-IR $\left(\mathbf{A T R}, \mathbf{c m}^{-1}\right): \tilde{v}=2963,2921,2872,2842,1744,1730,1504,1492,1480,1462,1454,1442,1398,1384$, $1366,1277,1231,1202,1194,1165,1118,1101,1068,1053,1030,1014,968,944,937,919,896,872,852$, $832,819,808,796,784,759,723,621,604,594,585,574,566,558$.

MS (EI, 70 eV): m/z (\%) = 343 (16), 342 (100), 257 (27), 230 (74), 217 (71), 130 (68), 129 (52), 116 (69), 83 (61), 55 (80).

HR-MS (EI, $70 \mathrm{eV}):\left[\mathrm{C}_{16} \mathrm{H}_{23} \mathrm{~F}_{5} \mathrm{~S}\right]$, calcd.: 342.1441; found: 342.1430.

tert-Butyl(3-((1R,2S,5R)-2-isopropyl-5-methylcyclohexyl)phenoxy)dimethylsilane (4h)

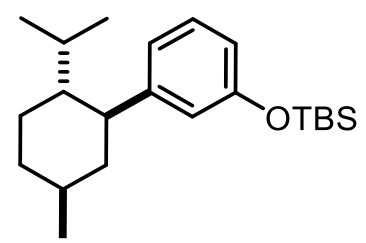

Isolated yield: $252 \mathrm{mg}, 0.37 \mathrm{mmol}, 73 \%$, colorless oil

dr: 99:1.

Purification: $i$-hexane

${ }^{1} \mathrm{H}-\mathrm{NMR}\left(400 \mathrm{MHz}_{\mathrm{CDCl}}, \mathrm{ppm}\right): \delta=7.30-7.21(\mathrm{~m}, 1 \mathrm{H}), 7.18-7.10(\mathrm{~m}, 1 \mathrm{H}), 6.87(\mathrm{dq}, J=7.7,0.9 \mathrm{~Hz}, 1 \mathrm{H}), 6.72-$ $6.64(\mathrm{~m}, 1 \mathrm{H}), 2.39(\mathrm{td}, J=11.5,3.4 \mathrm{~Hz}, 1 \mathrm{H}), 1.95-1.70(\mathrm{~m}, 3 \mathrm{H}), 1.62-1.37(\mathrm{~m}, 3 \mathrm{H}), 1.27-1.08(\mathrm{~m}, 3 \mathrm{H}), 1.02(\mathrm{dd}$, 
$J=1.7,0.7 \mathrm{~Hz}, 9 \mathrm{H}), 0.92(\mathrm{~d}, J=6.6 \mathrm{~Hz}, 3 \mathrm{H}), 0.85-0.80(\mathrm{~m}, 3 \mathrm{H}), 0.71(\mathrm{dd}, J=6.8,0.7 \mathrm{~Hz}, 3 \mathrm{H}), 0.22(\mathrm{dd}, J=2.7,0.7$ $\mathrm{Hz}, 6 \mathrm{H})$.

${ }^{13} \mathrm{C}-\mathrm{NMR}\left(100 \mathrm{MHz} \mathrm{CDCl}_{3}, \mathrm{ppm}\right): \delta=155.6,148.2,129.3,129.1,121.3,120.1,117.5,47.9,47.5,45.1,35.3$, $33.2,27.4,25.8,25.7,24.5,22.5,21.5,18.3,15.4,-4.4,-4.4$.

FT-IR (ATR, cm $^{-1}$ ): $\tilde{v}=2955,2929,2859,1597,1491,1484,1440,1362,1252,1155,1002,971,908,867,835$ $779,730,801,665$.

MS (EI, 70 eV): m/z (\%) = 346 (71), 290 (31), 289 (98), 193 (30), 179 (91), 165 (37), 75 (8).

HR-MS (EI, $70 \mathrm{eV}$ ): [ $\mathrm{C}_{22} \mathrm{H}_{38} \mathrm{OSi}$ ], calcd.: 346.2692; found: 346.2687.

1-((1R,2S,5R)-2-Isopropyl-5-methylcyclohexyl)naphthalene (4i)

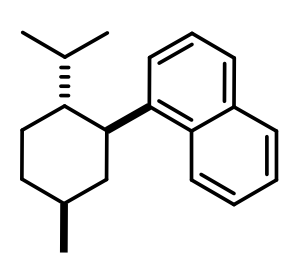

Isolated yield: $165 \mathrm{mg}, 0.31 \mathrm{mmol}, 62 \%$, colorless oil

dr: 99:1.

Purification: $i$-hexane

${ }^{1} \mathrm{H}-\mathrm{NMR}\left(400 \mathrm{MHz}^{\mathrm{CDCl}}{ }_{3}, \mathrm{ppm}\right): \delta=8.20-8.15(\mathrm{~m}, 1 \mathrm{H}), 7.84(\mathrm{dd}, J=8.0,1.5 \mathrm{~Hz}, 1 \mathrm{H}), 7.67(\mathrm{dd}, J=8.2,1.1 \mathrm{~Hz}$, 1H), $7.53-7.42(\mathrm{~m}, 3 \mathrm{H}), 7.38(\mathrm{dd}, J=7.3,1.3 \mathrm{~Hz}, 1 \mathrm{H}), 3.38(\mathrm{td}, J=11.5,3.4 \mathrm{~Hz}, 1 \mathrm{H}), 1.95-1.83(\mathrm{~m}, 4 \mathrm{H}), 1.77(\mathrm{tt}$, $J=11.5,3.0 \mathrm{~Hz}, 1 \mathrm{H}), 1.38-1.23(\mathrm{~m}, 2 \mathrm{H}), 1.13-1.04(\mathrm{~m}, 2 \mathrm{H}), 0.89(\mathrm{~d}, J=6.6 \mathrm{~Hz}, 3 \mathrm{H}), 0.81(\mathrm{~d}, J=7.0 \mathrm{~Hz}, 3 \mathrm{H}), 0.61$ $(\mathrm{d}, J=6.9 \mathrm{~Hz}, 3 \mathrm{H})$.

${ }^{13} \mathrm{C}-\mathrm{NMR}\left(100 \mathrm{MHz} \mathrm{CDCl}_{3}, \mathrm{ppm}\right): \delta=142.7,134.0,131.8,128.9,125.7,125.6,125.5,125.1,123.2,122.7,46.8$, $45.8,40.8,35.4,33.5,27.3,24.8,22.4,21.6,16.1$.

FT-IR (ATR, $\left.\mathbf{c m}^{-1}\right): \tilde{v}=3045,2925,2867,1595,1511,1455,1368,1016,905,794,776,729$.

MS (El, 70 eV): $m / z(\%)=266(100), 181$ (86), 154 (31), 142 (60), 141 (87), 69 (12).

HR-MS (EI, $70 \mathrm{eV}):$ [ $\left.\mathrm{C}_{20} \mathrm{H}_{26}\right]$, calcd.: 266.2035; found: 266.2020.

5-((1S,2R,5S)-2-Isopropyl-5-methylcyclohexyl)-1-methyl-1H-indole (4j) 


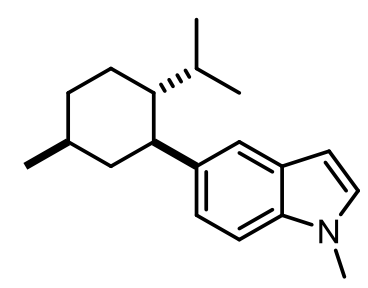

Isolated yield: $128 \mathrm{mg}, 0.38 \mathrm{mmol}, 75 \%$, white solid

dr: $96: 4$

Purification: $i$-hexane: ethyl acetate 99:1

${ }^{1} \mathrm{H}-\mathrm{NMR}\left(400 \mathrm{MHz}^{\mathrm{CDCl}} \mathrm{CD}_{3} \mathrm{ppm}\right): \delta=7.44-7.38(\mathrm{~m}, 1 \mathrm{H}), 7.28-7.23(\mathrm{~m}, 1 \mathrm{H}), 7.07(\mathrm{dd}, J=8.4,1.6 \mathrm{~Hz}, 1 \mathrm{H}), 7.02$ (d, J = $3.1 \mathrm{~Hz}, 1 \mathrm{H}), 6.43(\mathrm{dd}, J=3.1,0.9 \mathrm{~Hz}, 1 \mathrm{H}), 3.77(\mathrm{~s}, 3 \mathrm{H}), 2.52(\mathrm{td}, J=11.6,3.4 \mathrm{~Hz}, 1 \mathrm{H}), 1.89-1.78(\mathrm{~m}, 2 \mathrm{H})$, $1.77(\mathrm{dq}, J=12.4,3.1 \mathrm{~Hz}, 1 \mathrm{H}), 1.57-1.43(\mathrm{~m}, 3 \mathrm{H}), 1.30-1.11(\mathrm{~m}, 2 \mathrm{H}), 0.91(\mathrm{~d}, J=6.5 \mathrm{~Hz}, 3 \mathrm{H}), 0.79(\mathrm{~d}, J=6.9$ $\mathrm{Hz}, 4 \mathrm{H}), 0.70(\mathrm{~d}, J=6.8 \mathrm{~Hz}, 3 \mathrm{H})$.

${ }^{13} \mathrm{C}-\mathrm{NMR}\left(100 \mathrm{MHz}, \mathrm{CDCl}_{3}, \mathrm{ppm}\right): \delta=137.6,135.4,128.7,128.6,121.3,119.1,108.9,100.5,48.0,47.8,46.2$, $35.5,33.5,32.9,27.4,24.7,22.6,21.7,15.4$.

FT-IR $\left(A T R, \mathbf{c m}^{-1}\right): \tilde{v}=2957,2859,1600,1585,1484,1471,1443,1344,1278,1253,1159,1097,1001,908,837$, $781,726,700,662$.

MS (EI, 70 eV): m/z (\%) = 270 (15), 269 (82), 185 (13), 184 (100), 169 (6), 168 (5), 158 (9), 157 (11), 145 (25), $144(52), 131(7)$.

HR-MS (EI, $70 \mathrm{eV}$ ): [ $\mathrm{C}_{19} \mathrm{H}_{27} \mathrm{~N}$ ], calcd.: 269.2143; found: 269.2138.

2-((1R,2R)-2-(4-Methoxyphenyl)cyclohexyl)-5,5-dimethyl-1,3-dioxane (5a)<smiles>COc1ccc([C@H]2CCCC[C@H]2C2OCC(C)(C)CO2)cc1</smiles>

Isolated yield: $246 \mathrm{mg}, 0.41 \mathrm{mmol}, 81 \%$, colorless oil

dr: 99:1.

Purification: $i$-hexane: ethyl acetate $=95: 5$ 
${ }^{1} \mathrm{H}-\mathrm{NMR}\left(400 \mathrm{MHz}, \mathrm{CDCl}_{3}, \mathrm{ppm}\right): \delta=7.10(\mathrm{~d}, J=8.6 \mathrm{~Hz}, 2 \mathrm{H}), 6.83(\mathrm{~d}, J=8.7 \mathrm{~Hz}, 1 \mathrm{H}), 3.89(\mathrm{~d}, J=2.1 \mathrm{~Hz}, 2 \mathrm{H}), 3.80$ $(\mathrm{s}, 3 \mathrm{H}), 3.50(\mathrm{dd}, J=10.9,2.8 \mathrm{~Hz}, 2 \mathrm{H}), 3.15(\mathrm{dd}, J=10.9,1.0 \mathrm{~Hz}, 2 \mathrm{H}), 2.49(\mathrm{td}, J=11.9,3.5 \mathrm{~Hz}, 1 \mathrm{H}), 2.07$ (ddd, $J=$ $13.4,3.3,1.6 \mathrm{~Hz}, 1 \mathrm{H}), 1.88-1.66(\mathrm{~m}, 4 \mathrm{H}), 1.51-1.26(\mathrm{~m}, 4 \mathrm{H}), 1.13(\mathrm{~s}, 3 \mathrm{H}), 0.60(\mathrm{~s}, 3 \mathrm{H})$.

${ }^{13} \mathrm{C}-\mathrm{NMR}\left(100 \mathrm{MHz}, \mathrm{CDCl}_{3}, \mathrm{ppm}\right): \delta=157.7,137.8,128.4,113.6,101.6,55.1,47.6,44.7,35.4,30.1,26.7,25.94$, 24.3, 22.8, 21.6.

FT-IR $\left(A T R, \mathbf{c m}^{-1}\right): \tilde{v}=2931,2853,2248,1512,1394,1247,114,968,904,828,727,648$.

MS (El, $70 \mathrm{eV}): m / z(\%)=304$ (10), 200 (30), 196 (43), 17 (10), 120 (28), 114 (100), 69 (52), 45 (13), 41 (12).

HR-MS (EI, $70 \mathrm{eV}$ ): [ $\left.\mathrm{C}_{19} \mathrm{H}_{28} \mathrm{O}_{3}\right]$, calcd.: 304.2038; found: 304.2030.

5,5-Dimethyl-2-((1R,2R)-2-(4-(trifluoromethyl)phenyl)cyclohexyl)-1,3-dioxane (5b)

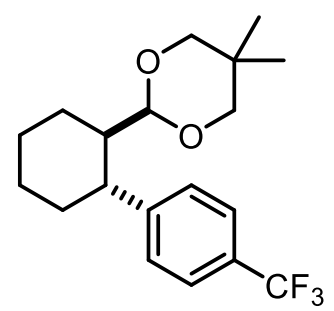

Isolated yield: $242 \mathrm{mg}, 0.36 \mathrm{mmol}, 71 \%$, colorless oil

dr: 99:1.

Purification: $i$-hexane:ethyl acetate $=95: 5$

${ }^{1} \mathrm{H}-\mathrm{NMR}\left(600 \mathrm{MHz}, \mathrm{CDCl}_{3}, \mathrm{ppm}\right): \delta=7.56-7.51(\mathrm{~m}, 2 \mathrm{H}), 7.29(\mathrm{~d}, J=8.0 \mathrm{~Hz}, 2 \mathrm{H}), 3.84(\mathrm{~d}, J=2.2 \mathrm{~Hz}, 1 \mathrm{H}), 3.49$ (ddd, $J=30.7,10.9,2.8 \mathrm{~Hz}, 2 \mathrm{H}$ ), 3.13 (ddd, $J=67.3,10.9,1.0 \mathrm{~Hz}, 2 \mathrm{H}$ ), $2.63(\mathrm{td}, J=11.9,3.3 \mathrm{~Hz}, 1 \mathrm{H}$ ), 2.10 (ddt, $J=$ $13.1,4.1,2.9 \mathrm{~Hz}, 1 \mathrm{H}), 1.90-1.76(\mathrm{~m}, 4 \mathrm{H}), 1.54-1.31(\mathrm{~m}, 4 \mathrm{H}), 1.11(\mathrm{~s}, 3 \mathrm{H}), 0.60(\mathrm{~s}, 3 \mathrm{H})$.

${ }^{13} \mathrm{C}-\mathrm{NMR}\left(100 \mathrm{MHz}, \mathrm{CDCl}_{3}, \mathrm{ppm}\right): \delta=149.8,127.9,126.4,125.2,104.3,101.2,47.1,45.5,35.1,30.1,26.5,25.8$, 24.2, 22.7, 21.5 .

FT-IR $\left(\right.$ ATR, cm $\left.^{-1}\right): \tilde{v}=2933,2856,2252,1618,1325,112,1116,1069,903,834,723,649,606$.

MS (EI, 70 eV): m/z (\%) = 341 (10), $196(23), 158(15), 115$ (100), 69 (47), 44 (12), 40 (13).

HR-MS (EI, $70 \mathrm{eV}$ ): [ $\mathrm{C}_{19} \mathrm{H}_{25} \mathrm{~F}_{3} \mathrm{O}_{2}$ ], calcd.: 342.1807; found: 342.1721.

tert-Butyldimethyl(3-((1R,2R,5R)-5-methyl-2-(prop-1-en-2-yl)cyclohexyl)phenoxy)silane (6a) 


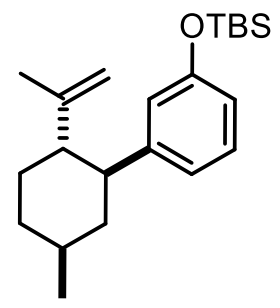

Isolated yield: $264 \mathrm{mg}, 0.39 \mathrm{mmol}, 77 \%$, colorless oil

dr: 99:1.

Purification: $i$-hexane:ethyl acetate $=95: 5$

${ }^{1} \mathrm{H}-\mathrm{NMR}\left(400 \mathrm{MHz}^{\mathrm{CDCl}} \mathrm{CDpm}_{3}\right.$ ): $\delta=7.16-7.06(\mathrm{~m}, 1 \mathrm{H}), 6.79-6.70(\mathrm{~m}, 1 \mathrm{H}), 6.64(\mathrm{dq}, J=8.8,1.3 \mathrm{~Hz}, 2 \mathrm{H}), 4.59-$ $4.52(\mathrm{~m}, 2 \mathrm{H}), 2.52(\mathrm{td}, J=11.6,3.4 \mathrm{~Hz}, 1 \mathrm{H}), 2.22(\mathrm{td}, J=11.6,3.3 \mathrm{~Hz}, 1 \mathrm{H}), 1.92-1.72(\mathrm{~m}, 3 \mathrm{H}), 1.67-1.28(\mathrm{~m}$, 6H), $1.24-1.06(\mathrm{~m}, 2 \mathrm{H}), 1.04-0.98(\mathrm{~m}, 7 \mathrm{H}), 0.94(\mathrm{~d}, J=6.6 \mathrm{~Hz}, 2 \mathrm{H}), 0.89(\mathrm{~d}, J=6.7 \mathrm{~Hz}, 2 \mathrm{H}), 0.20(\mathrm{~d}, J=0.6 \mathrm{~Hz}$, $6 \mathrm{H})$.

${ }^{13} \mathrm{C}-\mathrm{NMR}\left(100 \mathrm{MHz}, \mathrm{CDCl}_{3}, \mathrm{ppm}\right): \delta=155.4,148.3,147.7,128.8,120.6,119.4,117.5,111.1,51.1,47.9$, $44.5,41.4,35.1,33.1,32.8,25.7,22.5,19.8,18.2,11.5,-4.4,-4.4$.

FT-IR $\left(\right.$ ATR, cm $\left.{ }^{-1}\right): \tilde{v}=3072,2951,2926,2857,1645,1601,1483,1440,1374,1270,1252,1156,1002,982,936$, $907,866,779,731,698$.

MS (EI, 70 eV): m/z (\%) = 344 (63), 288 (38), 287 (100), 191 (33), 177 (93), 163 (41), 73 (14).

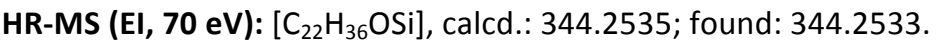

1-((1R,2R,5R)-5-Methyl-2-(prop-1-en-2-yl)cyclohexyl)-4-(trifluoromethyl)benzene (6b)

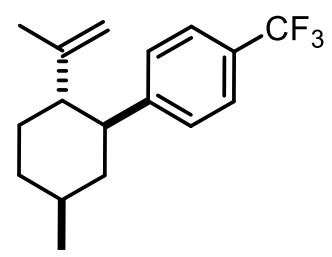

Isolated yield: $169 \mathrm{mg}, 0.30 \mathrm{mmol}, 60 \%$, colorless oil

dr: 99:1.

Purification: $i$-hexane

${ }^{1} \mathrm{H}-\mathrm{NMR}\left(400 \mathrm{MHz}^{\mathrm{CDCl}}{ }_{3}, \mathrm{ppm}\right): \delta=7.42(\mathrm{~d}, J=8.0 \mathrm{~Hz}, 2 \mathrm{H}), 7.16(\mathrm{~d}, J=8.0 \mathrm{~Hz}, 2 \mathrm{H}), 4.49-4.41(\mathrm{~m}, 2 \mathrm{H}), 2.57(\mathrm{td}$, $J=11.7,3.4 \mathrm{~Hz}, 1 \mathrm{H}), 2.20(\mathrm{td}, J=11.6,3.3 \mathrm{~Hz}, 1 \mathrm{H}), 1.74(\mathrm{dq}, J=13.4,3.3 \mathrm{~Hz}, 3 \mathrm{H}), 1.56-1.35(\mathrm{~m}, 5 \mathrm{H}), 1.13-0.95$ $(\mathrm{m}, 2 \mathrm{H}), 0.85(\mathrm{~d}, J=6.5 \mathrm{~Hz}, 3 \mathrm{H})$. 
${ }^{13} \mathrm{C}-\mathrm{NMR}\left(100 \mathrm{MHz}, \mathrm{CDCl}_{3}, \mathrm{ppm}\right): \delta=150.2,147.8,128.1,127.8,127.7,125.1,125.1,125.0,111.6,50.8,47.8$, $44.5,35.0,323.0,32.6,22.4,19.7$.

FT-IR $\left(A T R, \mathbf{c m}^{-1}\right): \tilde{v}=2927,2873,1463,1381,1326,1168,1131,1069,957,890,739,564$.

MS (El, 70 eV): $m / z(\%)=282(16), 218(35), 216(100), 148(32), 123$ (28), 91 (36), 83 (23), 57 (22).

HR-MS (EI, $70 \mathrm{eV}):\left[\mathrm{C}_{17} \mathrm{H}_{21} \mathrm{~F}_{3}\right]$, calcd.: 282.1595; found: 282.1570.

1-Methoxy-4-((1R,2R,5R)-5-methyl-2-(prop-1-en-2-yl)cyclohexyl)benzene (6c)

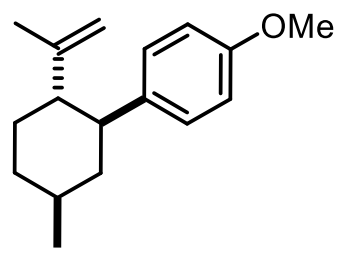

Isolated yield: $104 \mathrm{mg}, 0.43 \mathrm{mmol}, 85 \%$, colorless oil

dr: 99:1.

Purification: $i$-hexane:ethyl acetate $=100: 1$

${ }^{1} \mathrm{H}-\mathrm{NMR}\left(400 \mathrm{MHz}, \mathrm{CDCl}_{3}, \mathrm{ppm}\right): \delta=7.08-7.06(\mathrm{~m}, 1 \mathrm{H}), 7.05(\mathrm{~d}, J=2.2 \mathrm{~Hz}, 1 \mathrm{H}), 6.81(\mathrm{~d}, J=2.2 \mathrm{~Hz}, 1 \mathrm{H}), 6.79(\mathrm{~d}$, $J=2.1 \mathrm{~Hz}, 1 \mathrm{H}), 4.61-4.46(\mathrm{~m}, 2 \mathrm{H}), 3.78(\mathrm{~s}, 2 \mathrm{H}), 2.52(\mathrm{td}, J=11.7,3.4 \mathrm{~Hz}, 1 \mathrm{H}), 2.23(\mathrm{td}, J=11.6,3.4 \mathrm{~Hz}, 1 \mathrm{H}), 1.80$ (dddd, $J=11.3,9.6,4.9,2.4 \mathrm{~Hz}, 3 \mathrm{H}), 1.64-1.42(\mathrm{~m}, 6 \mathrm{H}), 1.22-1.02(\mathrm{~m}, 2 \mathrm{H}), 0.92(\mathrm{~d}, J=6.5 \mathrm{~Hz}, 2 \mathrm{H}), 0.91-0.85$ $(\mathrm{m}, 1 \mathrm{H})$.

${ }^{13} \mathrm{C}-\mathrm{NMR}\left(100 \mathrm{MHz}, \mathrm{CDCl}_{3}, \mathrm{ppm}\right): \delta=157.5,148.6,138.3,129.4,128.2,113.9,113.4,111.0,55.1,51.1,47.0$, $45.1,35.1,33.1,32.8,22.4,19.7$.

FT-IR (ATR, cm $\left.^{-1}\right)$ : $\tilde{v}=3070,2948,2916,1645,1610,1511,1454,1373,1301,1243,1177,1037,906,885,826$, $754,729,692$.

MS (EI, 70 eV): $m / z(\%)=244$ (44), 229 (11), 173 (26), 161 (100), 134 (33), 121 (92), 91 (23), 55(23).

HR-MS (EI, $70 \mathrm{eV}):\left[\mathrm{C}_{17} \mathrm{H}_{24} \mathrm{O}\right]$, calcd.: 244.1827; found: 244.1823.

1-((1S,2S,4S)-2-(4-methoxyphenyl)-4-methylcyclohexyl)ethan-1-one (7) 


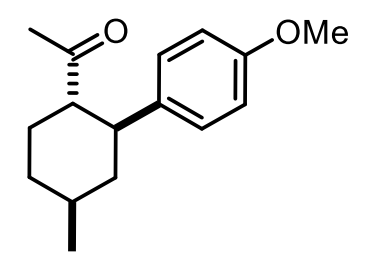

Substrate $6 \mathrm{c}(244 \mathrm{mg}, 1 \mathrm{mmol})$ and was dissolved in dry $\mathrm{CH}_{2} \mathrm{Cl}_{2}(15 \mathrm{~mL})$ and the solution was cooled to $-78^{\circ} \mathrm{C}$, at which point a stream of $\mathrm{O}_{3}$ was introduced through a disposable pipet for $3 \mathrm{~min}$. After $15 \mathrm{~min}$, the reaction was sparged with $\mathrm{O}_{2}$ and then argon. The crude reaction mixture was diluted with $\mathrm{CH}_{2} \mathrm{Cl}_{2}(10 \mathrm{~mL})$ and sat. aq. $\mathrm{NaHCO}_{3}(15 \mathrm{~mL})$. The aqueous layer was extracted with $\mathrm{CH}_{2} \mathrm{Cl}_{2}(3 \times 5 \mathrm{~mL})$ and the combined organic layers were dried over $\mathrm{Na}_{2} \mathrm{SO}_{4}$ and filtered. The residue was purified via flash chromatography with hexanes/ethyl acetate 100:1 to furnish the ketone 7 as a white solid (150 $\mathrm{mg}, 0.61 \mathrm{mmol}, 61 \%$ ).

dr: 99:1.

m.p.: $102.8-104.5^{\circ} \mathrm{C}$

${ }^{1} \mathrm{H}-\mathrm{NMR}\left(400 \mathrm{MHz}^{\mathrm{CDCl}}{ }_{3}, \mathrm{ppm}\right): \delta=7.01(\mathrm{~d}, J=8.6 \mathrm{~Hz}, 2 \mathrm{H}), 6.73(\mathrm{~d}, J=8.7 \mathrm{~Hz}, 2 \mathrm{H}), 3.69(\mathrm{~s}, 3 \mathrm{H}), 2.61-2.65(\mathrm{~m}$, $2 \mathrm{H}), 1.89-1.68(\mathrm{~m}, 6 \mathrm{H}), 1.58-1.34(\mathrm{~m}, 2 \mathrm{H}), 1.18-0.91(\mathrm{~m}, 2 \mathrm{H}), 0.86(\mathrm{~d}, J=6.5 \mathrm{~Hz}, 3 \mathrm{H})$.

${ }^{13} \mathrm{C}-\mathrm{NMR}\left(100 \mathrm{MHz}, \mathrm{CDCl}_{3}, \mathrm{ppm}\right): \delta=212.8,157.9,136.7,128.2,113.9,57.3,55.2,45.6,43.3,34.1,32.6,30.1$, $29.5,22.4$

FT-IR (ATR, $\mathbf{c m}^{-1}$ ): $\tilde{v}=2924,2253,1706,1610,1512,1455,1355,1245,1178,1035,905,827,726$.

MS (EI, 70 eV): m/z (\%) = 246 (77), 161 (100), 121 (84), 97 (28), 71 (28), 69 (34), 57 (54), 55 (48), 43 (44), 42 (58).

HR-MS (EI, $70 \mathrm{eV}$ ): [ $\mathrm{C}_{16} \mathrm{H}_{22} \mathrm{O}_{2}$ ], calcd.: 246.1620; found: 246.1621.

(3S,4R)-3-Isopropyl-4-(4-(trifluoromethyl)phenyl)tetrahydrofuran (8a)

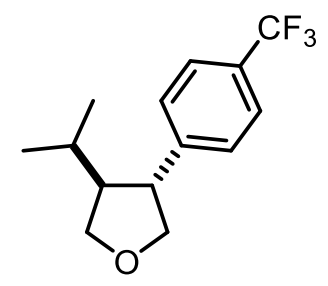

Isolated yield: $92 \mathrm{mg}, 0.36 \mathrm{mmol}, 71 \%$, colorless liquid

dr: $97: 3$.

Purification: $i$-hexane:ethyl acetate $=95: 5$ 
${ }^{1} \mathrm{H}-\mathrm{NMR}\left(300 \mathrm{MHz}, \mathrm{CDCl}_{3}, \mathrm{ppm}\right): \delta=7.55(\mathrm{~d}, J=8.1 \mathrm{~Hz}, 2 \mathrm{H}), 7.38(\mathrm{~d}, J=8.3 \mathrm{~Hz}, 2 \mathrm{H}), 4.18(\mathrm{dd}, J=8.7,7.8 \mathrm{~Hz}$, $1 \mathrm{H}$ ), 4.11 (ddd, $J=8.9,7.8,0.5 \mathrm{~Hz}, 1 \mathrm{H}$ ), 3.74 (dd, $J=8.9,6.7 \mathrm{~Hz}, 1 \mathrm{H}$ ), $3.64(\mathrm{t}, J=8.5 \mathrm{~Hz}, 1 \mathrm{H}$ ), $3.14(\mathrm{q}, J=7.3 \mathrm{~Hz}$, $1 \mathrm{H}), 2.16(\mathrm{p}, J=7.8 \mathrm{~Hz}, 1 \mathrm{H}), 1.79-1.60(\mathrm{~m}, 1 \mathrm{H}), 0.88(\mathrm{~d}, J=6.7 \mathrm{~Hz}, 3 \mathrm{H}), 0.84(\mathrm{~d}, J=6.7 \mathrm{~Hz}, 3 \mathrm{H})$.

${ }^{13} \mathrm{C}-\mathrm{NMR}\left(75 \mathrm{MHz}, \mathrm{CDCl}_{3}, \mathrm{ppm}\right): \delta=148.4(\mathrm{q}, J=1.8,1.1 \mathrm{~Hz}), 128.7(\mathrm{q}, J=32.5 \mathrm{~Hz}), 127.9,125.5(\mathrm{q}, J=3.8 \mathrm{~Hz})$, $124.0(q, J=271.8 \mathrm{~Hz}), 76.3,72.6,50.0,31.3,21.6,20.5$.

FT-IR (ATR, cm $\left.^{-1}\right): \tilde{v}=2962,2874,1620,1469,1423,1390,1370,1323,1242,1188,1163,1119,1110,1089$, $1067,1018,950,927,836,761,716,670,647,637,605,576,566,556$.

MS (EI, 70 eV): m/z (\%) = 258 (4), 228 (6), 213 (9), 186 (7), 185 (23), 173 (10), 172 (100), 165 (7), 159 (9), 115 (8), 91 (5), 95 (6).

HR-MS (EI, $70 \mathrm{eV}):\left[\mathrm{C}_{14} \mathrm{H}_{17} \mathrm{~F}_{3} \mathrm{O}\right]$, calcd.: 258.1231; found: 258.1233.

Ethyl 3-((3R,4S)-4-isopropyltetrahydrofuran-3-yl)benzoate (8b)

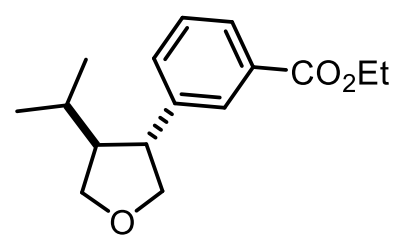

Isolated yield: $84 \mathrm{mg}, 0.32 \mathrm{mmol}, 64 \%$, colorless oil

dr: 99:1.

Purification: $i$-hexane:ethyl acetate $=95: 5$

${ }^{1} \mathrm{H}-\mathrm{NMR}\left(400 \mathrm{MHz}, \mathrm{CDCl}_{3}, \mathrm{ppm}\right): \delta=7.94(\mathrm{~s}, 1 \mathrm{H}), 7.89(\mathrm{~d}, J=7.8 \mathrm{~Hz}, 1 \mathrm{H}), 7.46(\mathrm{~d}, J=7.8 \mathrm{~Hz}, 1 \mathrm{H}), 7.37(\mathrm{t}, J=7.7$ $\mathrm{Hz}, 1 \mathrm{H}), 4.38(\mathrm{q}, J=7.3 \mathrm{~Hz}, 2 \mathrm{H}), 4.18(\mathrm{t}, J=8.4 \mathrm{~Hz}, 1 \mathrm{H}), 4.13(\mathrm{t}, J=8.3 \mathrm{~Hz}, 1 \mathrm{H}), 3.72(\mathrm{t}, J=8.0 \mathrm{~Hz}, 1 \mathrm{H}), 3.66(\mathrm{t}, J=$ $8.4 \mathrm{~Hz}, 1 \mathrm{H}), 3.14(\mathrm{q}, J=7.7 \mathrm{~Hz}, 1 \mathrm{H}), 2.20(\mathrm{p}, J=7.9 \mathrm{~Hz}, 1 \mathrm{H}), 1.78-1.61(\mathrm{~m}, J=6.8 \mathrm{~Hz}, 1 \mathrm{H}), 1.40(\mathrm{t}, J=7.2 \mathrm{~Hz}, 3 \mathrm{H})$, $0.88(\mathrm{~d}, J=6.7 \mathrm{~Hz}, 3 \mathrm{H}), 0.82(\mathrm{~d}, J=6.7 \mathrm{~Hz}, 3 \mathrm{H})$.

${ }^{13} \mathrm{C}-\mathrm{NMR}\left(100 \mathrm{MHz}, \mathrm{CDCl}_{3}, \mathrm{ppm}\right): \delta=166.7,144.1,132.2,130.8,128.7,128.7,127.6,72.6,61.1,55.0,50.4$, $31.3,21.7,20.5,14.4$.

FT-IR (ATR, cm $\left.^{-1}\right): \tilde{v}=2961,1618,1424,1323,1303,1185,1158,1122,1107,1094,1067,1048,1027,1016$, $1004,840,814,740,709,665,653$.

MS (EI, $70 \mathrm{eV}): m / z(\%)=262$ (17), 253 (14), 216 (28), 177 (22), 176 (100), 164 (21), 148 (27), 145 (14), 131 (37), 129 (12), 128 (11), 117 (50), $116(15), 115$ (33), 77 (17).

HR-MS (EI, $70 \mathrm{eV}$ ): [ $\mathrm{C}_{16} \mathrm{H}_{22} \mathrm{O}_{3}$ ], calcd.: 262.1569; found: 262.1562. 


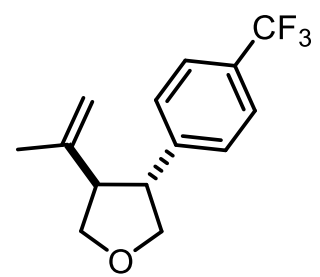

Isolated yield: $69 \mathrm{mg}, 0.27 \mathrm{mmol}, 54 \%$, colorless oil

dr: 99:1.

Purification: $i$-hexane:ethyl acetate $=9: 1$

${ }^{1} \mathrm{H}-\mathrm{NMR}\left(400 \mathrm{MHz}, \mathrm{CDCl}_{3}, \mathrm{ppm}\right): \delta=7.49(\mathrm{~d}, J=8.1 \mathrm{~Hz}, 2 \mathrm{H}), 7.29(\mathrm{~d}, J=8.1 \mathrm{~Hz}, 2 \mathrm{H}), 4.75-4.64(\mathrm{~m}, 1 \mathrm{H}), 4.68(\mathrm{~d}$, $J=0.8 \mathrm{~Hz}, 1 \mathrm{H}), 4.18(\mathrm{t}, J=8.3 \mathrm{~Hz}, 1 \mathrm{H}), 4.11(\mathrm{t}, J=8.3 \mathrm{~Hz}, 1 \mathrm{H}), 3.82-3.64(\mathrm{~m}, 2 \mathrm{H}), 3.30(\mathrm{q}, J=8.3 \mathrm{~Hz}, 1 \mathrm{H}), 2.94$ $(\mathrm{q}, J=8.6 \mathrm{~Hz}, 1 \mathrm{H}), 1.66(\mathrm{~s}, 3 \mathrm{H})$.

${ }^{13}$ C-NMR (100 MHz, CDCl 3 ppm): $\delta=145.6,142.5,129.0(q, J=32.4 \mathrm{~Hz}), 127.8,125.6(q, J=3.7 \mathrm{~Hz}), 124.2(q, J$ $=275.1 \mathrm{~Hz}), 112.6,75.2,72.6,55.1,49.5,20.3$.

FT-IR $\left(A T R, \mathbf{c m}^{-1}\right): \tilde{v}=2936,2863,1620,1423,1323,1163,1114,1067,1017,945,923,896,835$.

MS (EI, 70 eV): m/z (\%) = 256 (15), 237 (11), 212 (14), 211 (100), 198 (18), 192 (35), 188 (81), 177 (16), 172 (43), 159 (19), 157 (32), 142 (31), 141 (11).

HR-MS (EI, $70 \mathrm{eV}$ ): [ $\mathrm{C}_{14} \mathrm{H}_{15} \mathrm{~F}_{3} \mathrm{O}$ ], calcd.: 256.1075; found: 256.1068.

(3R,4R)-3-(4-Methoxyphenyl)-4-(prop-1-en-2-yl)tetrahydrofuran (8d)

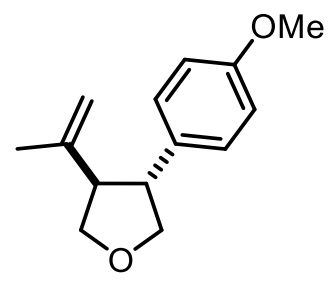

Isolated yield: $62 \mathrm{mg}, 0.29 \mathrm{mmol}, 57 \%$, colorless oil

dr: 99:1.

Purification: $i$-hexane: ethyl acetate $=95: 5$ 
${ }^{1} \mathrm{H}-\mathrm{NMR}\left(400 \mathrm{MHz}, \mathrm{CDCl}_{3}, \mathrm{ppm}\right): \delta=7.20-7.13(\mathrm{~m}, 2 \mathrm{H}), 6.88-6.83(\mathrm{~m}, 2 \mathrm{H}), 4.80-4.74(\mathrm{~m}, 2 \mathrm{H}), 4.23(\mathrm{t}, J=8.2$ $\mathrm{Hz}, 1 \mathrm{H}), 4.15(\mathrm{t}, J=8.3 \mathrm{~Hz}, 1 \mathrm{H}), 3.79(\mathrm{~s}, 3 \mathrm{H}), 3.78-3.73(\mathrm{~m}, 2 \mathrm{H}), 3.28(\mathrm{q}, J=8.8 \mathrm{~Hz}, 1 \mathrm{H}), 2.98(\mathrm{q}, J=8.7 \mathrm{~Hz}, 1 \mathrm{H})$, $1.72(\mathrm{~s}, 3 \mathrm{H})$.

${ }^{13} \mathrm{C}-\mathrm{NMR}\left(100 \mathrm{MHz}, \mathrm{CDCl}_{3}, \mathrm{ppm}\right): \delta=158.3,143.0,132.8,128.4,114.0,112.3,75.6,72.6,55.3,54.8,48.9,20.3$. FT-IR (ATR, $\left.\mathbf{c m}^{-1}\right)$ : $\tilde{v}=2936,2863,1620,1423,1323,1163,1114,1067,1017,945,923,896,835$.

MS (El, 70 eV): m/z (\%) = 218 (21), 203 (15), 173 (12), 150 (28), 135 (15), 134 (16), 122 (11), 121 (100), 43 (10). HR-MS (EI, $70 \mathrm{eV}$ ): [ $\mathrm{C}_{14} \mathrm{H}_{18} \mathrm{O}_{2}$ ], calcd.: 218.1307; found: 218.1304.

trans-3-Isopropyl-4-(3-methoxyphenyl)-1-tosylpyrrolidine (8e)

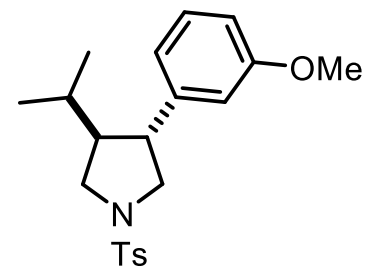

Isolated yield: $246 \mathrm{mg}, 0.33 \mathrm{mmol}, 66 \%$, colorless oil

dr: 99:1.

Purification: $i$-hexane:ethyl acetate $=95: 5$

${ }^{1} \mathrm{H}-\mathrm{NMR}\left(600 \mathrm{MHz}, \mathrm{CDCl}_{3}, \mathrm{ppm}\right): \delta=7.68-7.62(\mathrm{~m}, 2 \mathrm{H}), 7.29-7.24(\mathrm{~m}, 2 \mathrm{H}), 7.09(\mathrm{t}, J=7.9 \mathrm{~Hz}, 1 \mathrm{H}), 6.68-6.54$ $(\mathrm{m}, 4 \mathrm{H}), 3.68(\mathrm{~s}, 2 \mathrm{H}), 3.51(\mathrm{ddd}, J=39.1,9.9,8.0 \mathrm{~Hz}, 2 \mathrm{H}), 3.03(\mathrm{dt}, J=34.1,9.6 \mathrm{~Hz}, 2 \mathrm{H}), 2.81(\mathrm{td}, J=9.6,8.0 \mathrm{~Hz}$, 1H), $2.37(\mathrm{~s}, 3 \mathrm{H}), 2.00(\mathrm{tdd}, J=9.7,8.0,6.1 \mathrm{~Hz}, 1 \mathrm{H}), 1.52-1.39(\mathrm{~m}, 1 \mathrm{H}), 0.69(\mathrm{~d}, J=6.8 \mathrm{~Hz}, 3 \mathrm{H}), 0.64(\mathrm{~d}, J=6.8$ $\mathrm{Hz}, 3 \mathrm{H})$.

${ }^{13} \mathrm{C}-\mathrm{NMR}\left(100 \mathrm{MHz}, \mathrm{CDCl}_{3}, \mathrm{ppm}\right): \delta=159.8,143.5,142.5,133.6,129.7,127.6,119.9,113.7,111.8,55.9,55.2$, $51.7,50.5,48.1,29.2,21.5,18.7$.

FT-IR $\left(\right.$ ATR, cm $\left.^{-1}\right): \tilde{v}=2959,1598,1487,1466,1341,1287,1263,1156,1094,1042,1015,909,814,781,730$, 662.

MS (EI, $70 \mathrm{eV}): m / z(\%)=222$ (100), 219 (15), 190 (17), 146 (16), 134 (23), 121 (16), 110 (16), 91 (29), 83 (14), $42(56)$.

HR-MS (EI, $70 \mathrm{eV}):\left[\mathrm{C}_{21} \mathrm{H}_{27} \mathrm{NO}_{3} \mathrm{~S}\right]$, calcd.: 373.1712; found: 373.1709. 
trans-3-Isopropyl-4-(3-methoxyphenyl)-1-tosylpyrrolidine (8f)

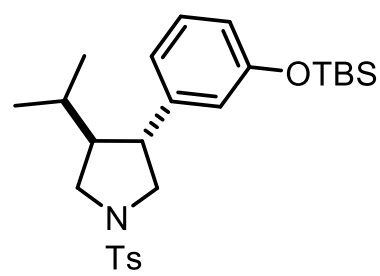

Isolated yield: $416 \mathrm{mg}, 0.44 \mathrm{mmol}, 88 \%$, colorless oil

dr: 99:1.

Purification: $i$-hexane:ethyl acetate $=95: 5$

${ }^{1} \mathrm{H}-\mathrm{NMR}\left(600 \mathrm{MHz}^{\mathrm{CDCl}}{ }_{3}, \mathrm{ppm}\right): \delta=7.69-7.63(\mathrm{~m}, 2 \mathrm{H}), 7.31-7.22(\mathrm{~m}, 2 \mathrm{H}), 7.02(\mathrm{t}, J=7.8 \mathrm{~Hz}, 1 \mathrm{H}), 6.60(\mathrm{ddt}, J$ = 8.0, 3.4, 1.3 Hz, 2H), $6.51(\mathrm{t}, J=2.0 \mathrm{~Hz}, 1 \mathrm{H}), 3.52$ (ddd, J=38.5, 9.9, 8.0 Hz, 2H), $3.02(\mathrm{dt}, J=28.5,9.7 \mathrm{~Hz}, 2 \mathrm{H}$ ), $2.78(\mathrm{td}, J=9.7,7.9 \mathrm{~Hz}, 1 \mathrm{H}), 2.37(\mathrm{~s}, 3 \mathrm{H}), 2.04-1.89(\mathrm{~m}, 1 \mathrm{H}), 1.45(\mathrm{dq}, J=13.4,6.7 \mathrm{~Hz}, 1 \mathrm{H}), 0.89(\mathrm{~s}, 9 \mathrm{H}), 0.69(\mathrm{~d}$, $J=6.7 \mathrm{~Hz}, 3 \mathrm{H}), 0.63(\mathrm{~d}, J=6.8 \mathrm{~Hz}, 3 \mathrm{H}), 0.09(\mathrm{~s}, 6 \mathrm{H})$.

${ }^{13} \mathrm{C}-\mathrm{NMR}\left(100 \mathrm{MHz}, \mathrm{CDCl}_{3}, \mathrm{ppm}\right): \delta=155.9,143.5,142.4,133.6,129.7,129.6,127.6,120.7,119.2,118.7,55.9$, $51.7,50.6,47.9,29.2,25.7,21.6,21.5,18.8,18.2,-4.4$

FT-IR (ATR, $\mathbf{c m}^{-1}$ ): $\tilde{v}=2957,2859,1600,1585,1484,1471,1443,1344,1278,1253,1159,1097,1001,908,837$, $781,726,700,662$.

MS (EI, 70 eV): m/z (\%) = 417 (26), 416 (100), 149 (13), 91 (4), 73 (3), 42 (6).

HR-MS (EI, 70 eV): [C $\left.{ }_{26} \mathrm{H}_{39} \mathrm{NO}_{3} \mathrm{SSi}\right]$, calcd.: 473.2420; found: 473.2329. 


\section{${ }^{1} \mathrm{H}$ NMR and ${ }^{13} \mathrm{C}$ NMR Spectra}

Triisopropyl(((1R,2S,5R)-5-methyl-2-(prop-1-en-2-yl)cyclohexyl)ethynyl)silane (3a)

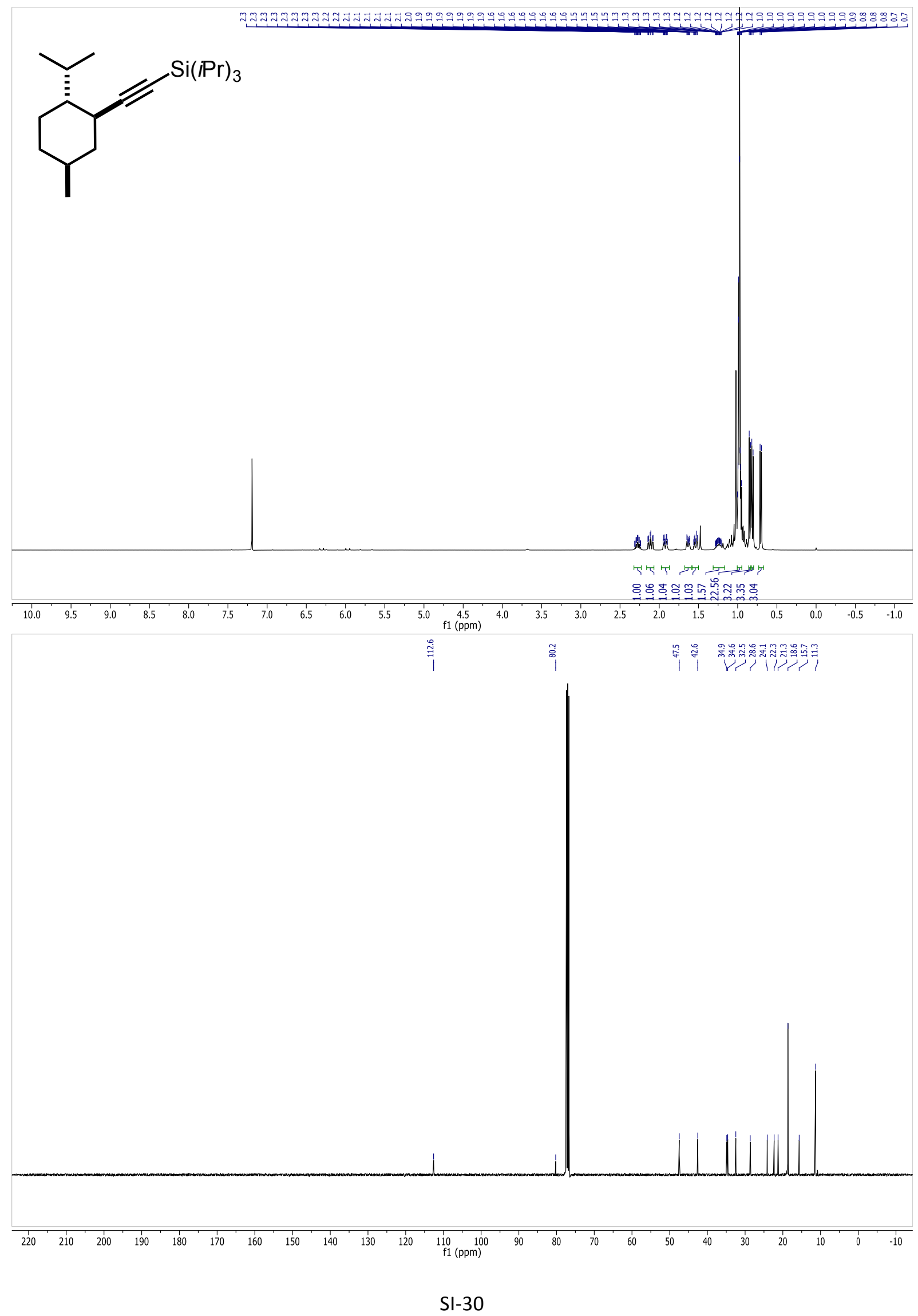


1-(((1R,2S,5R)-2-Isopropyl-5-methylcyclohexyl)ethynyl)-4-methoxybenzene (3b)
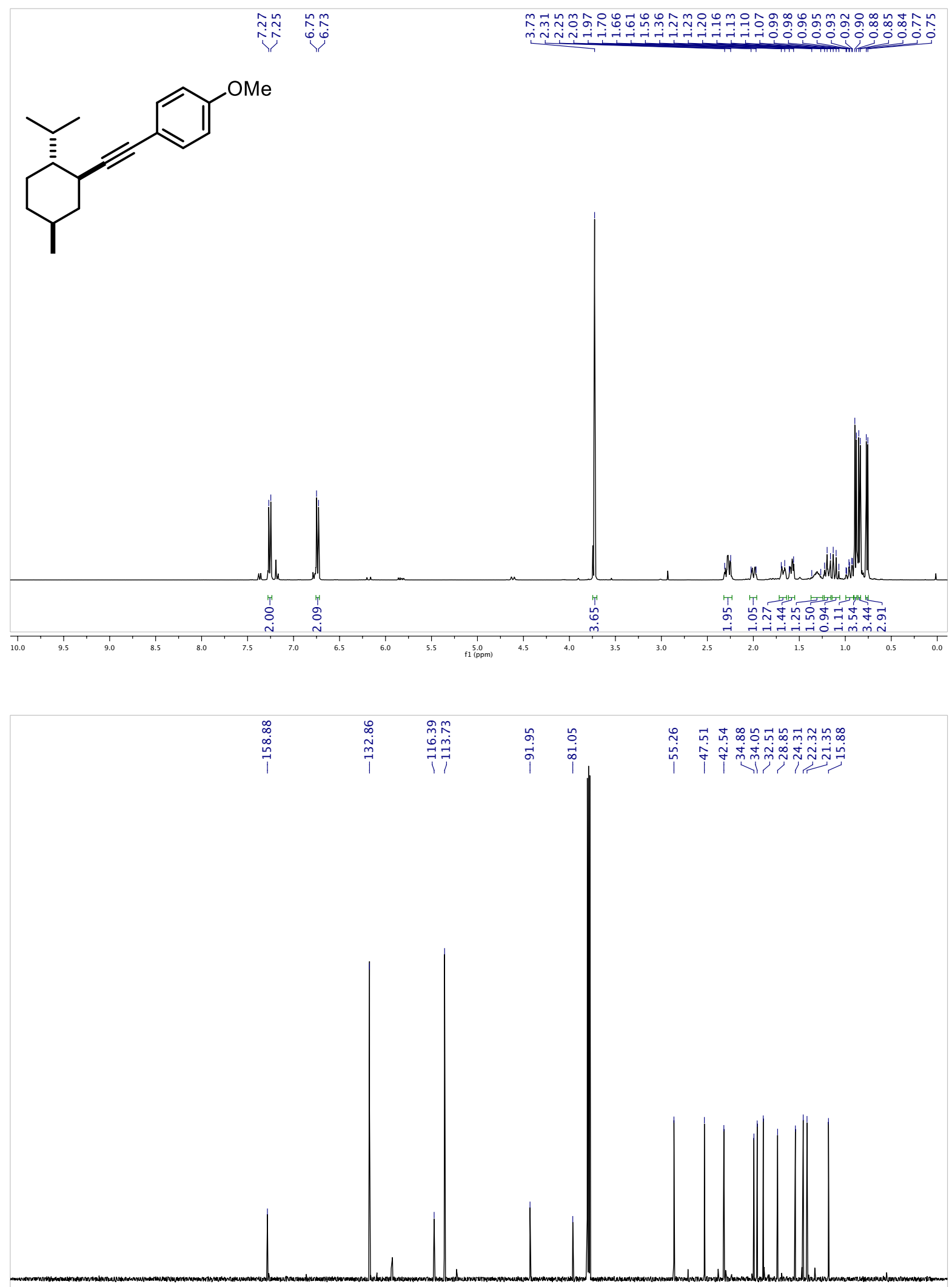
tert-Butyl(((1R,2S)-2-((4-methoxyphenyl)ethynyl)cyclopentyl)oxy)dimethylsilane (3c)
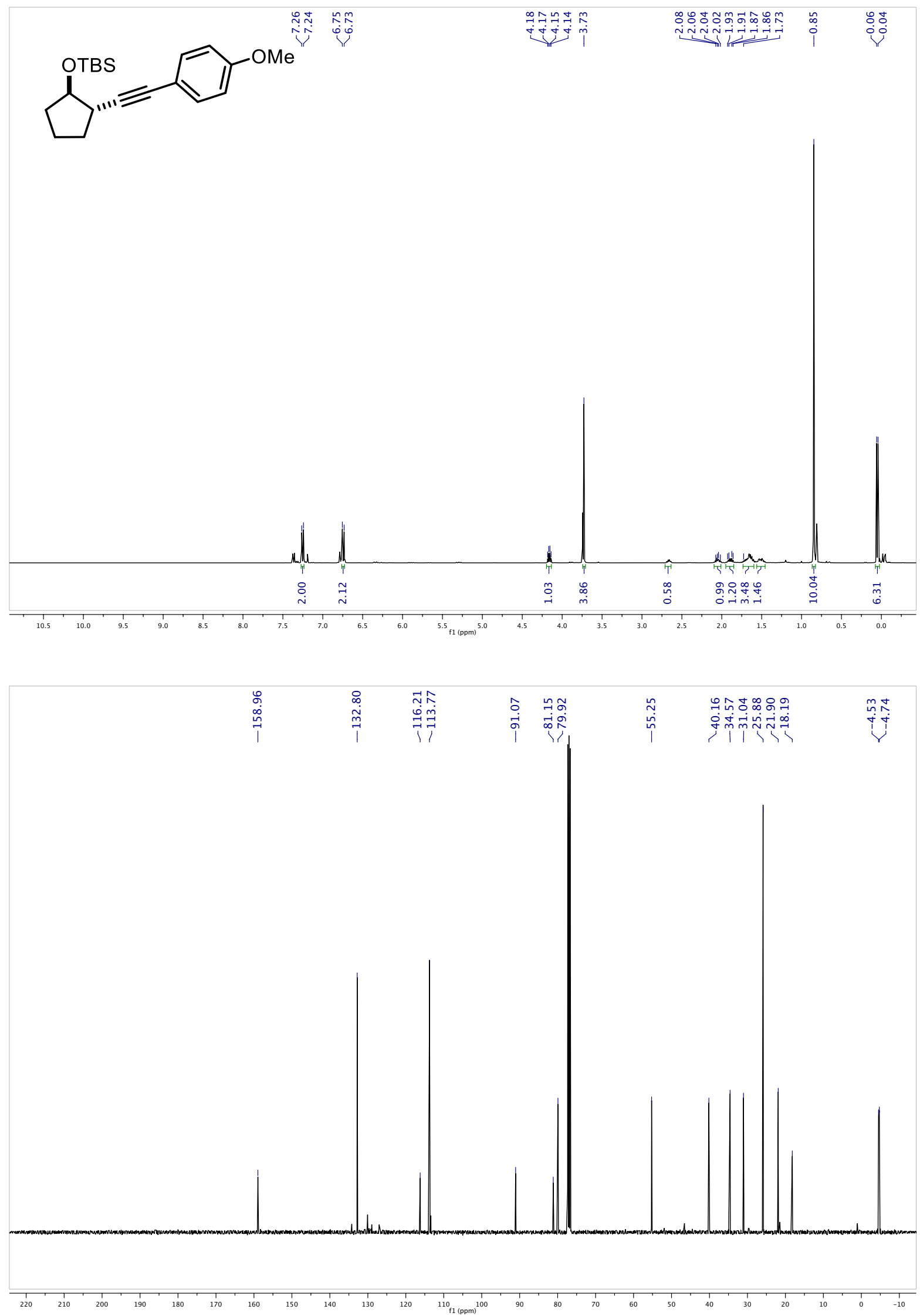
tert-Butyl(((1R,2S,5R)-2-isopropyl-5-methylcyclohexyl)ethynyl)dimethylsilane (3d)
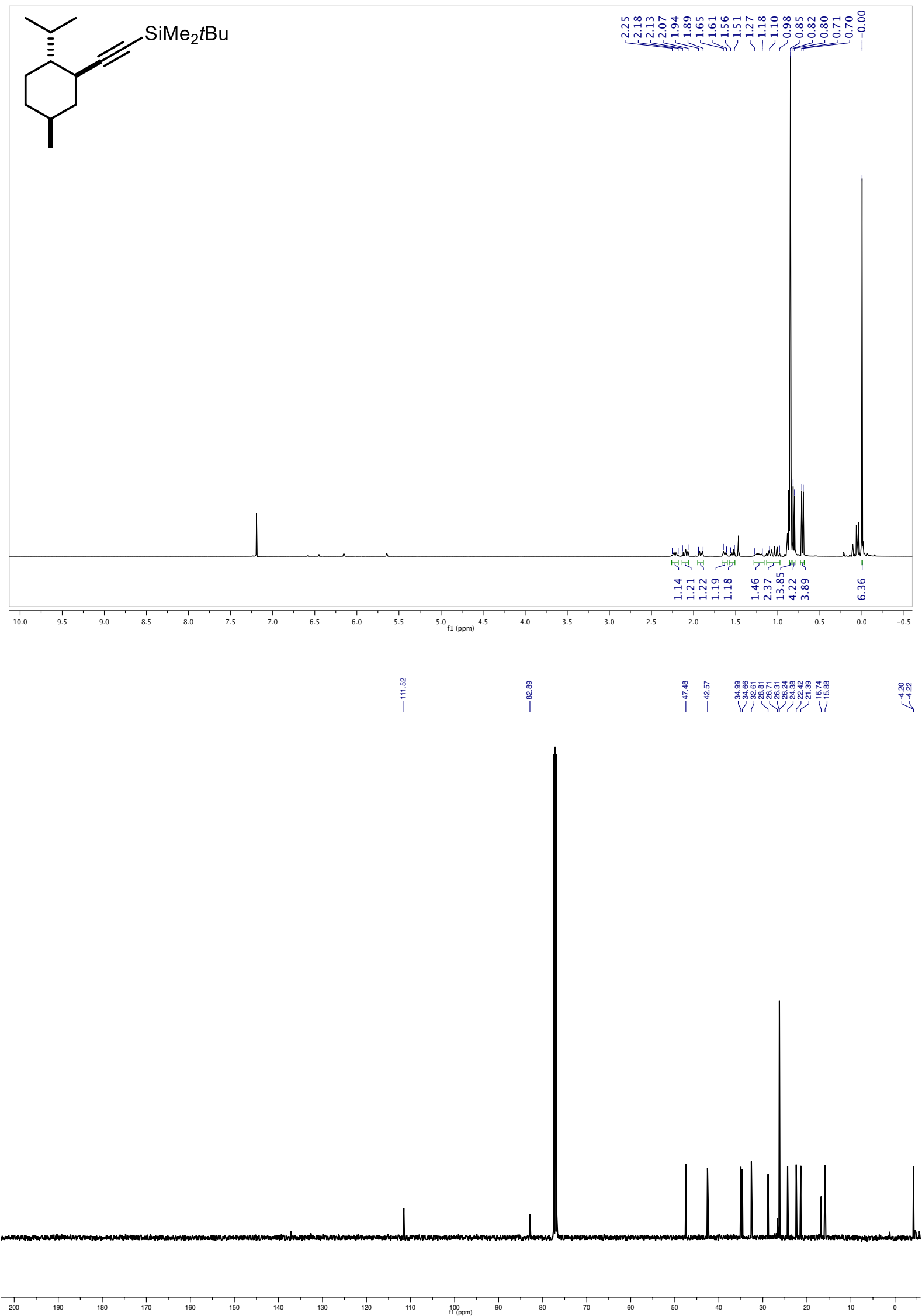
(((1S,2R,5S)-2-Isopropyl-5-methylcyclohexyl)ethynyl)trimethylsilane (3e)
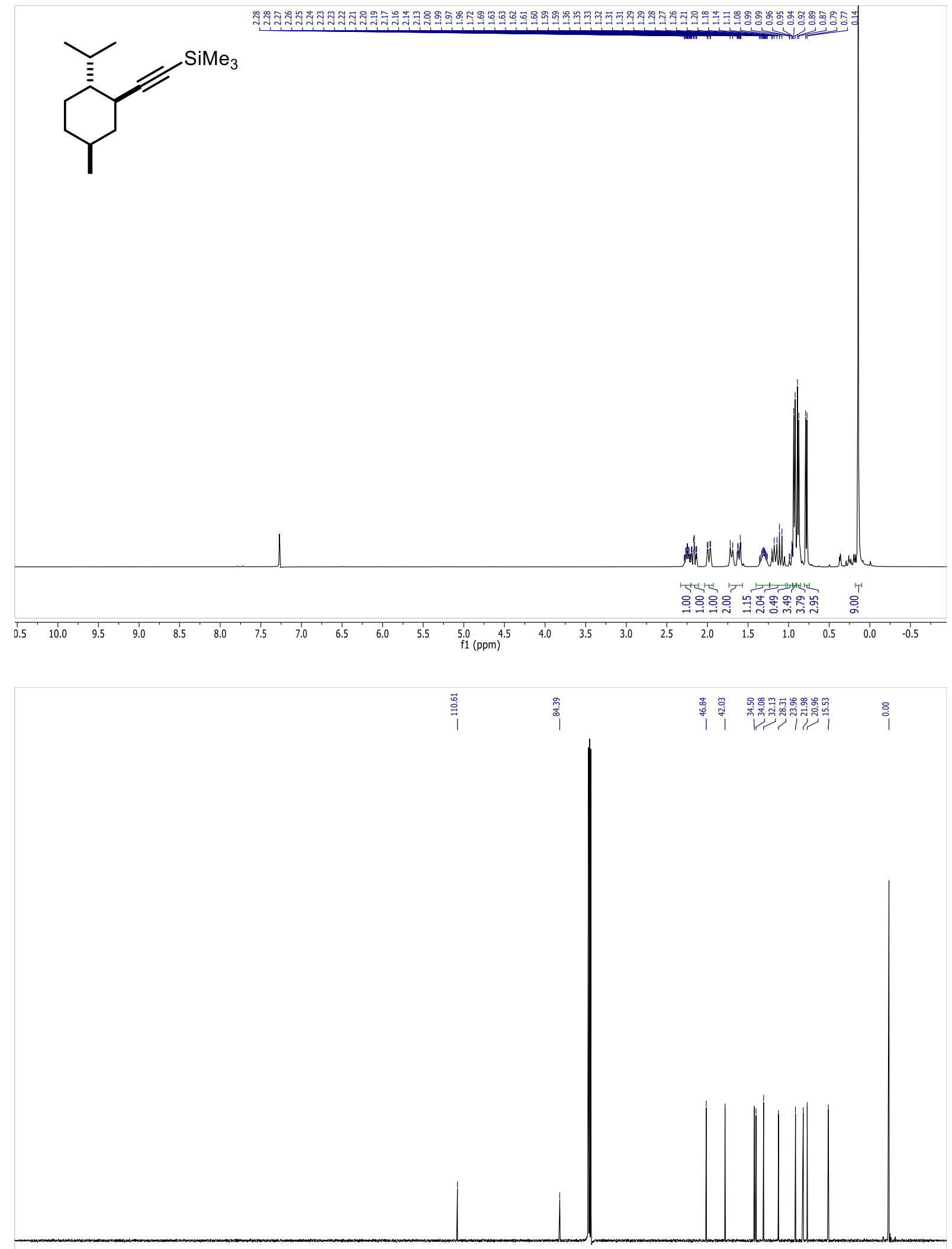

$\begin{array}{lllllllllllll}220 & 210 & 200 & 190 & 180 & 170 & 160 & 150 & 140 & 130 & 120 & 110 & 100 \\ \mathrm{f} 1(\mathrm{ppm})\end{array}$ 
tert-Butyldimethyl(((1R,2S,5R)-5-methyl-2-(prop-1-en-2-yl)cyclohexyl)ethynyl)silane (3f)

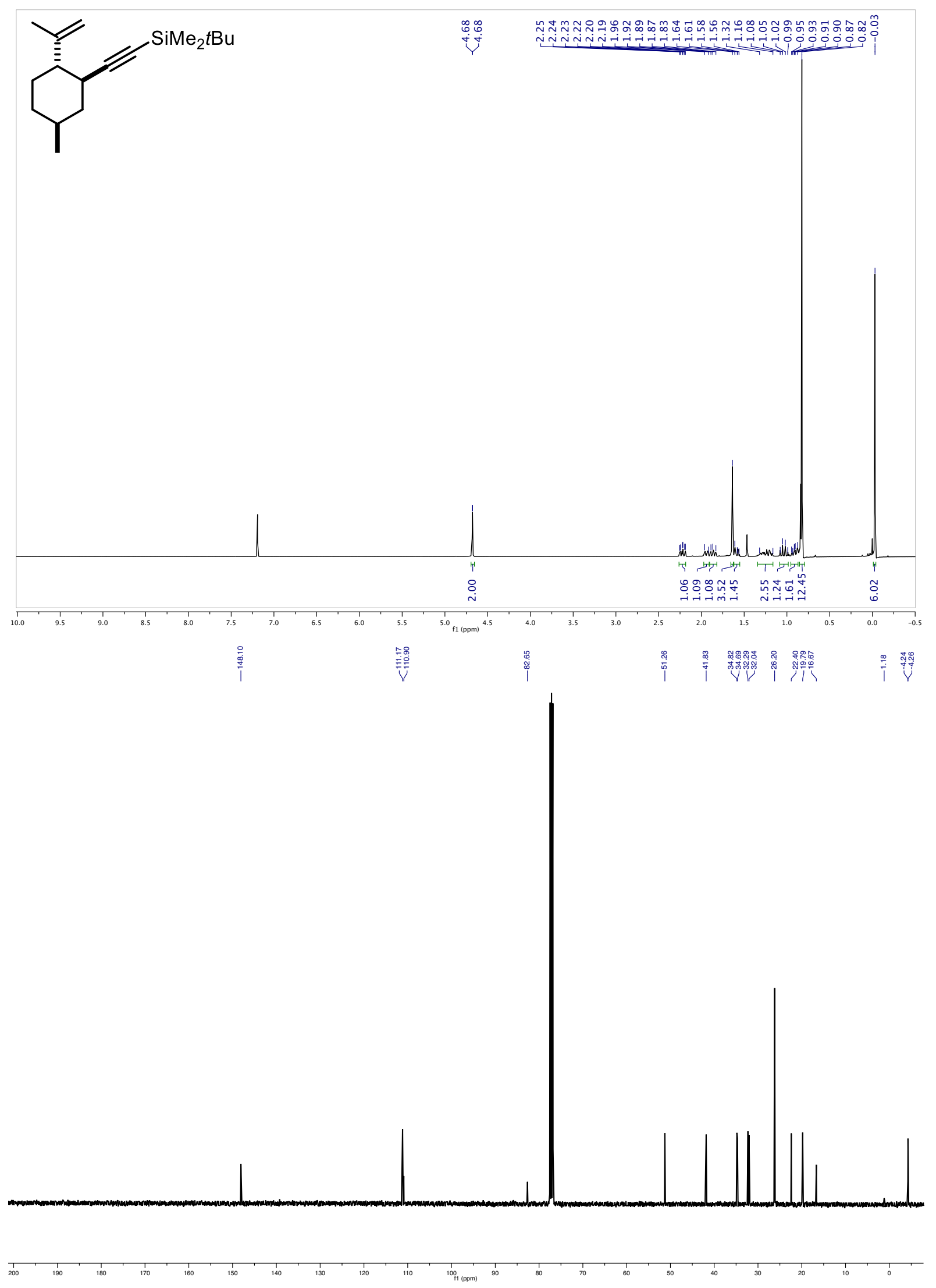

SI-35 
Triisopropyl(((1S,2S,5S)-5-methyl-2-(prop-1-en-2-yl)cyclohexyl)ethynyl)silane (3g)
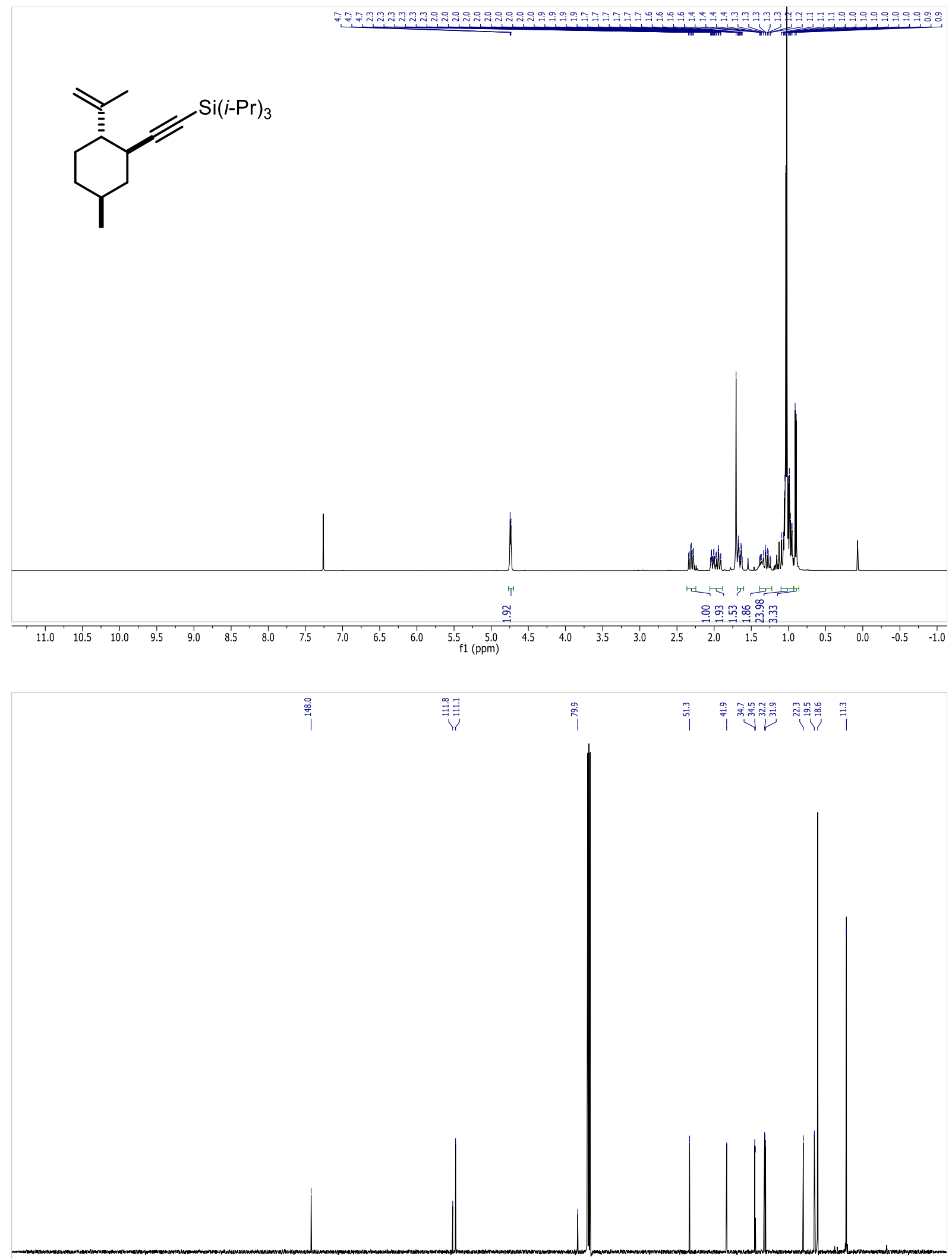

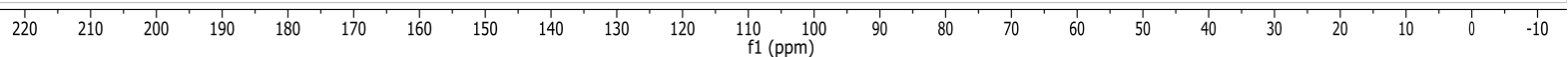


tert-Butyl(((1R,2S)-2-(cyclopentylethynyl)cyclopentyl)oxy)dimethylsilane (3h)

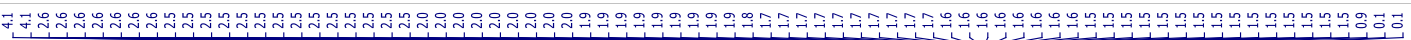

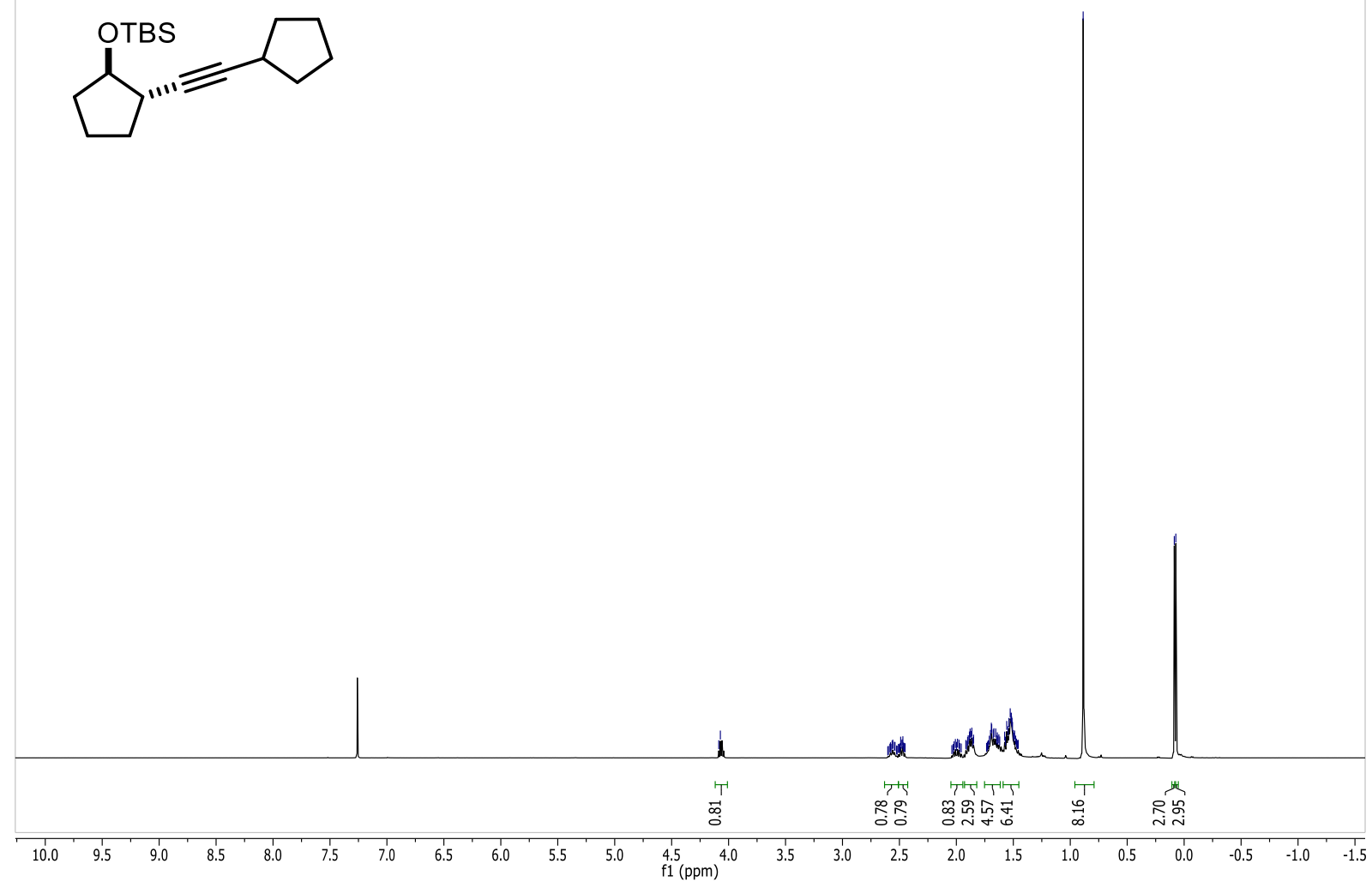

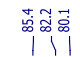

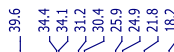

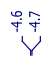

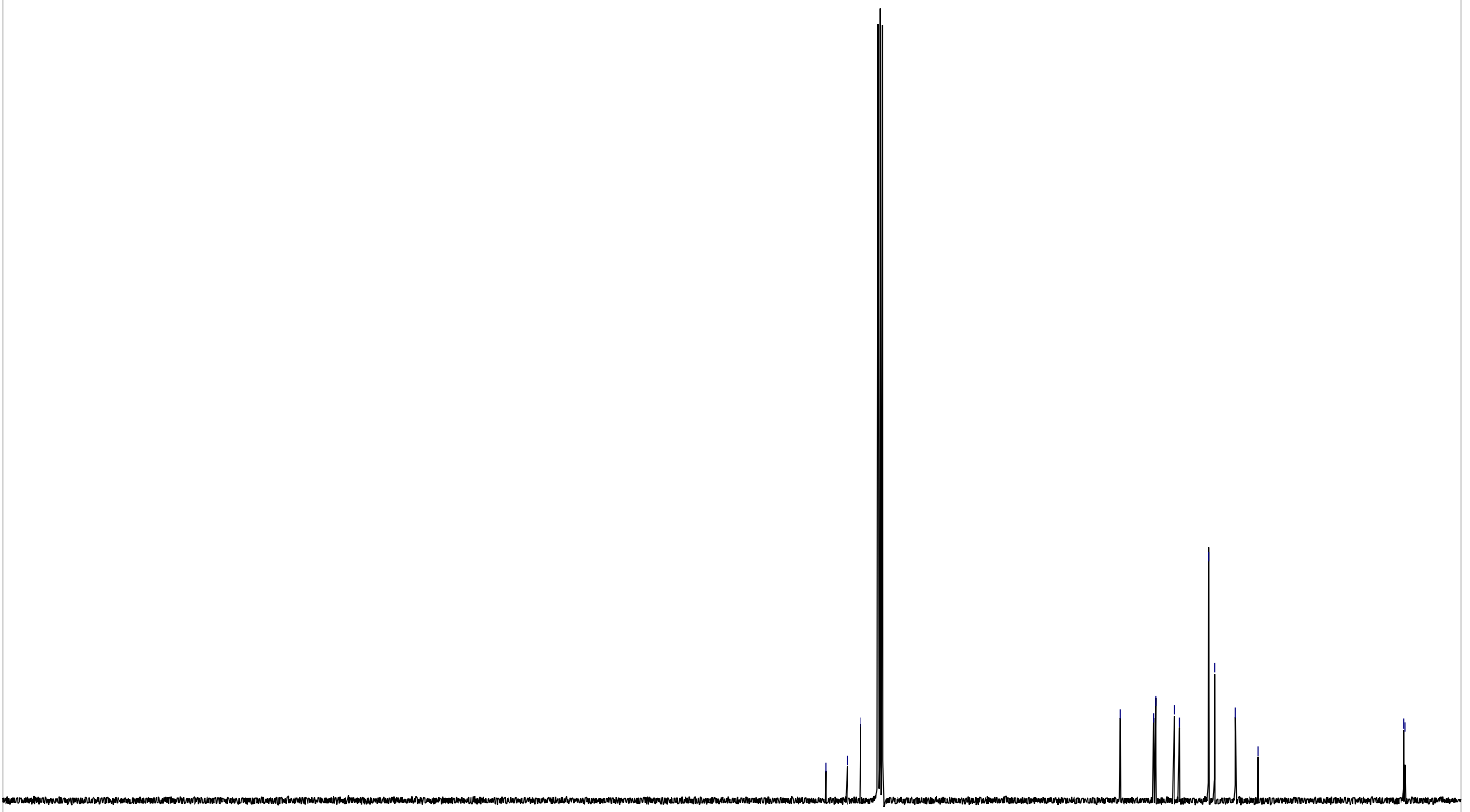

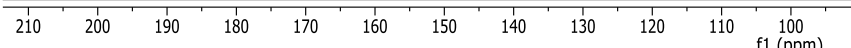

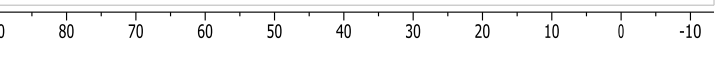


tert-butyl(((1R,2S)-2-(cyclohex-1-en-1-ylethynyl)cyclopentyl)oxy)dimethylsilane (3i)
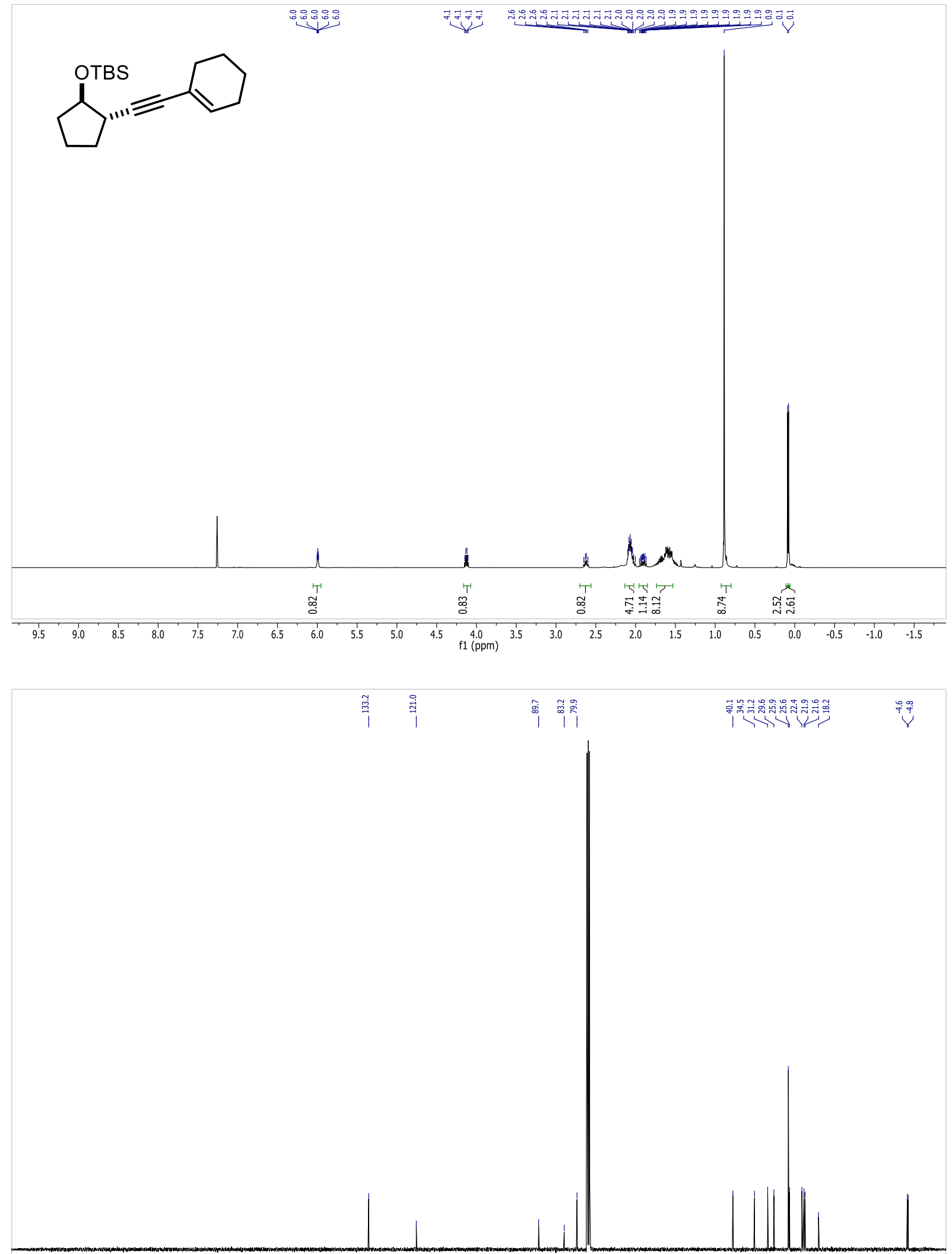

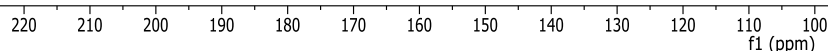


4-(((1S,2R)-2-((tert-butyldimethylsilyl)oxy)cyclohexyl)ethynyl)benzonitrile (3j)

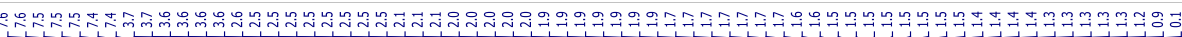<smiles>N#Cc1ccc(C#C[C@@H]2CCCC[C@@H]2[OH2+])cc1</smiles>
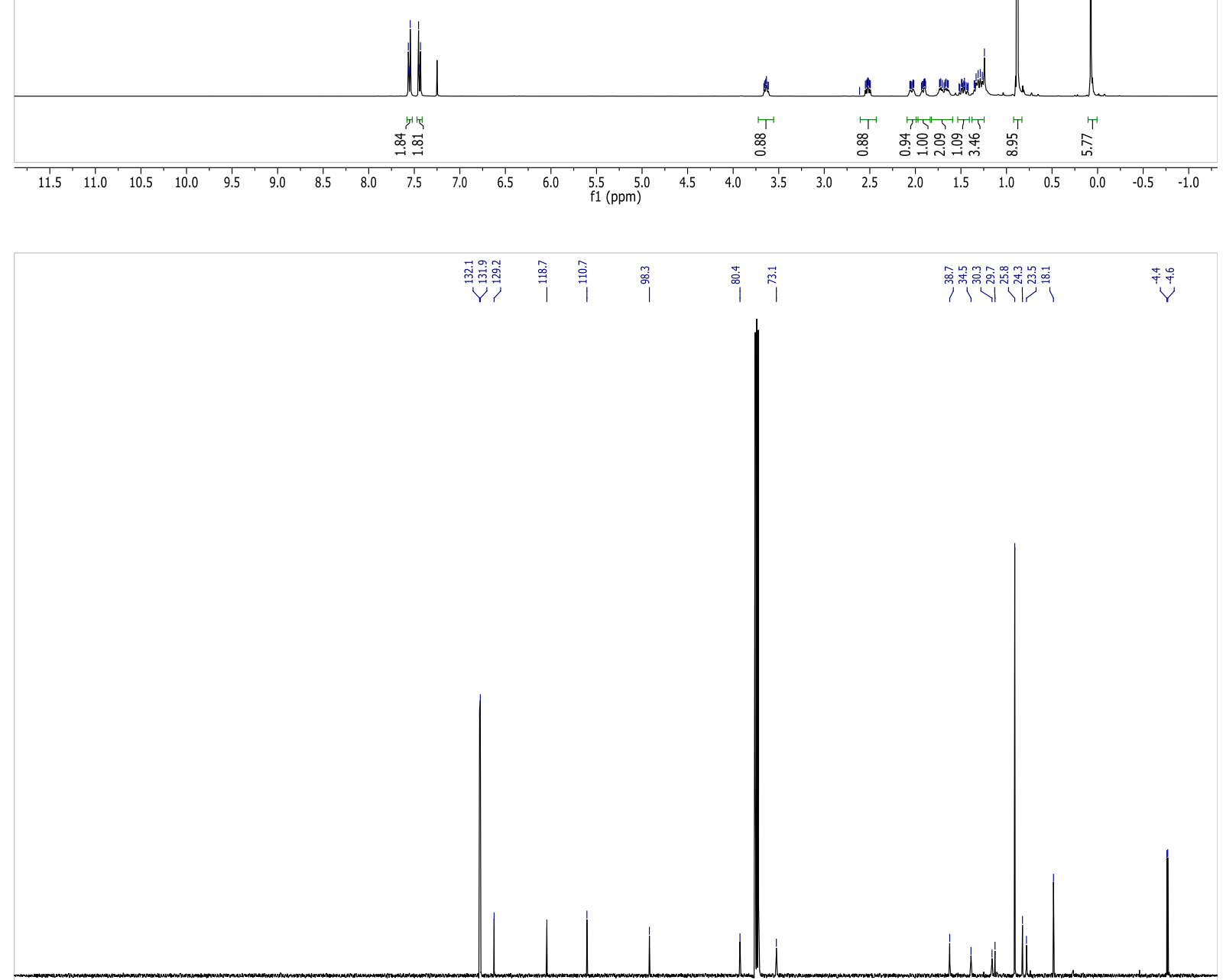

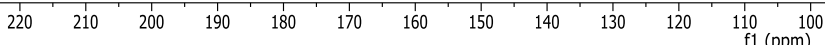


tert-Butyldimethyl((trans-2-((triisopropylsilyl)ethynyl)cyclohexyl)oxy)silane (3k)
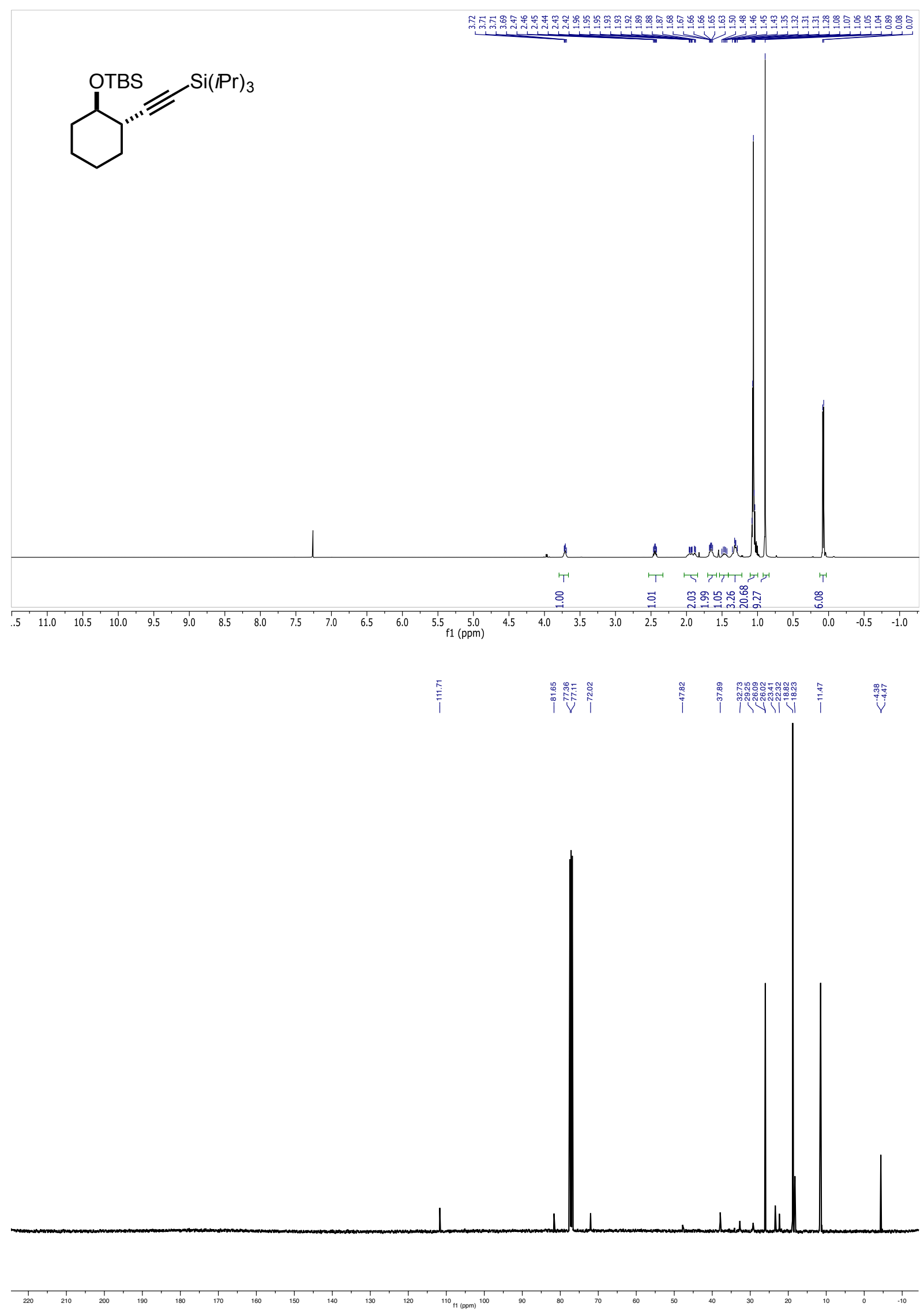
1-((1R,2S,5R)-2-Isopropyl-5-methylcyclohexyl)-4-(trifluoromethyl)benzene (4a)

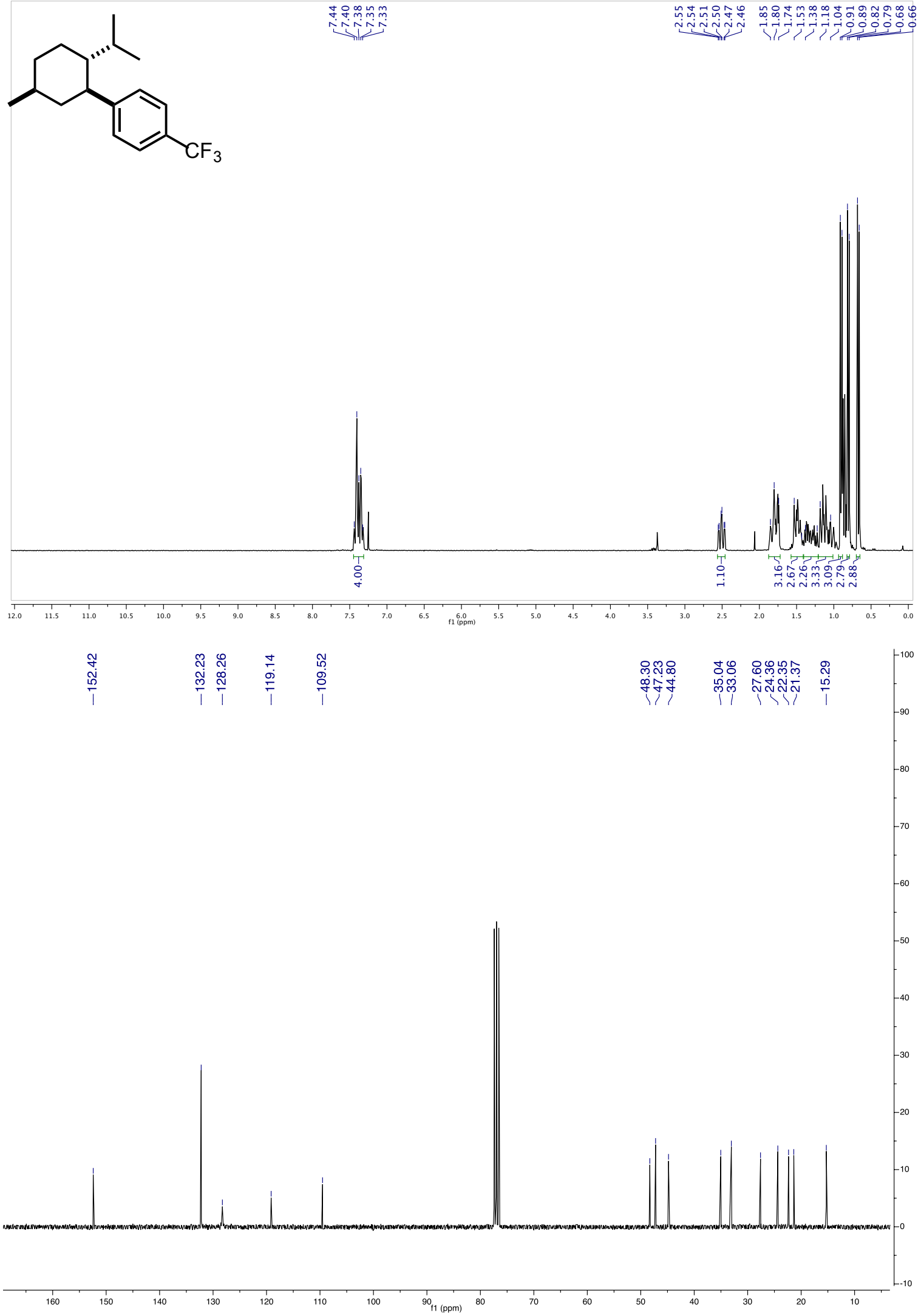


1-(4-((1R,2S,5R)-2-Isopropyl-5-methylcyclohexyl)phenyl)-2,2-dimethylpropan-1-one (4b)
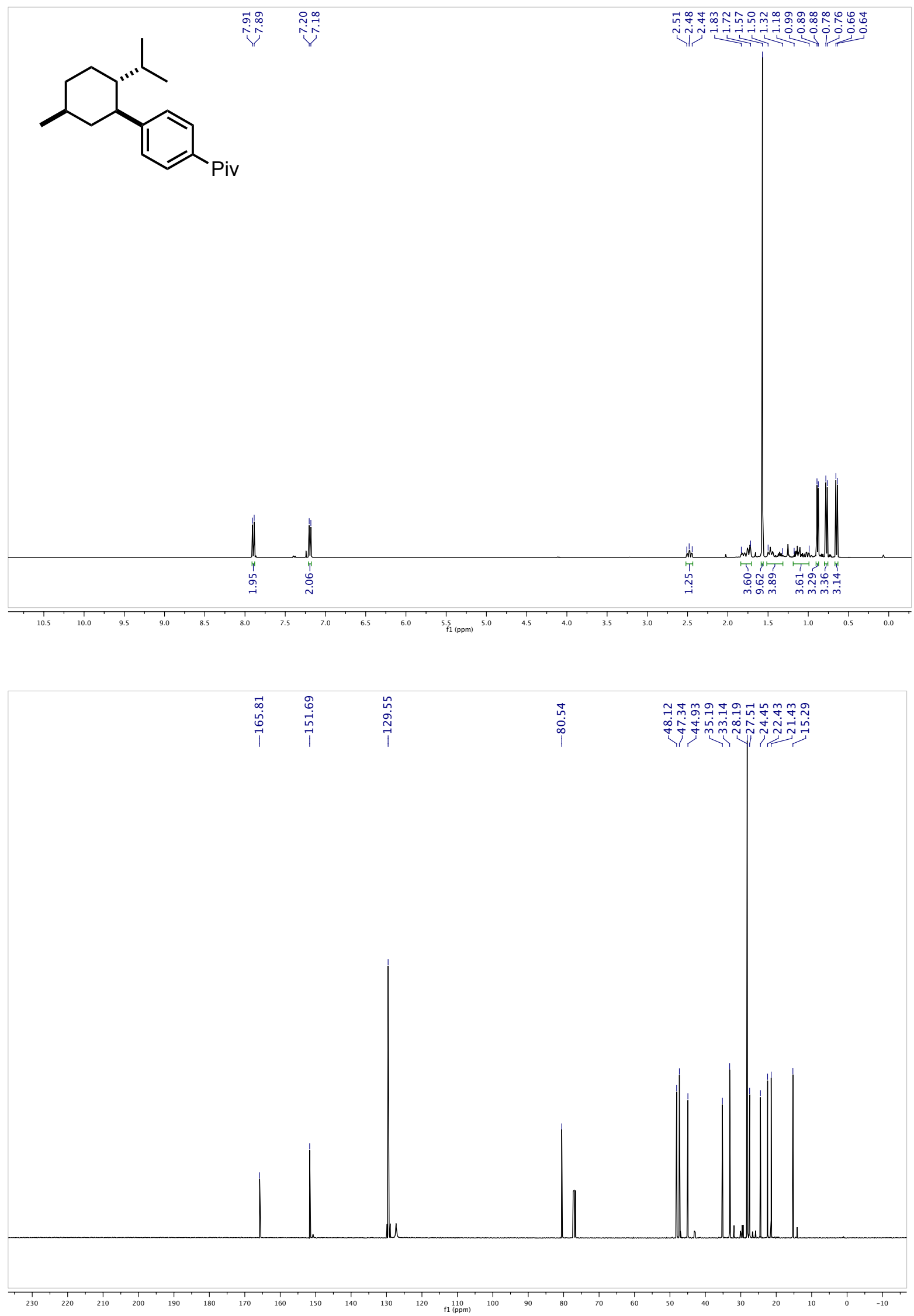
4-((1R,2S,5R)-2-Isopropyl-5-methylcyclohexyl)benzonitrile (4c)
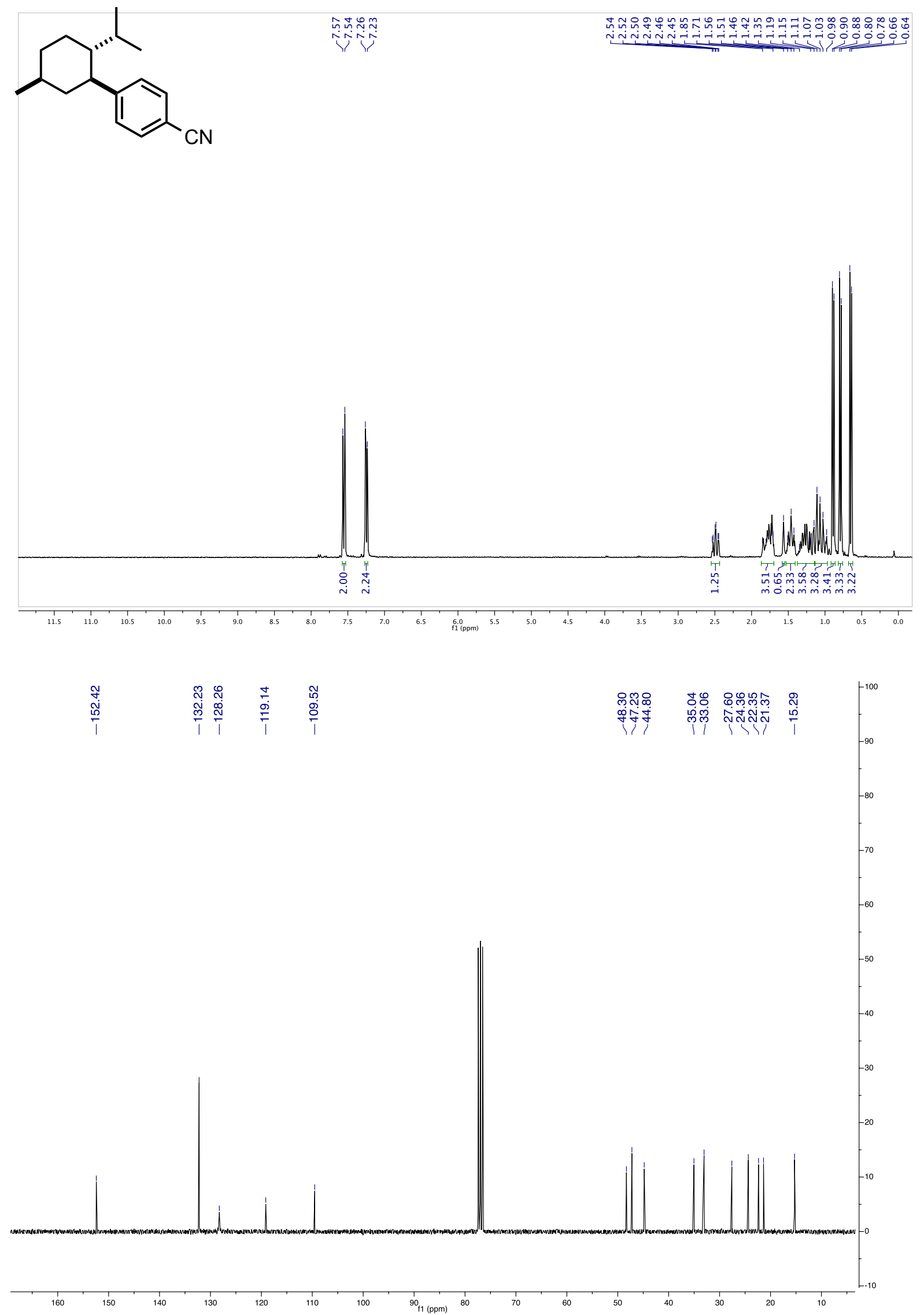
4-((1S,2R,5S)-2-Isopropyl-5-methylcyclohexyl)phenyl pivalate (4d)<smiles>CC(C)[C@H]1CC[C@@H](C)C[C@H]1c1ccc(O[Na])cc1</smiles>

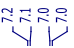

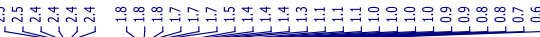
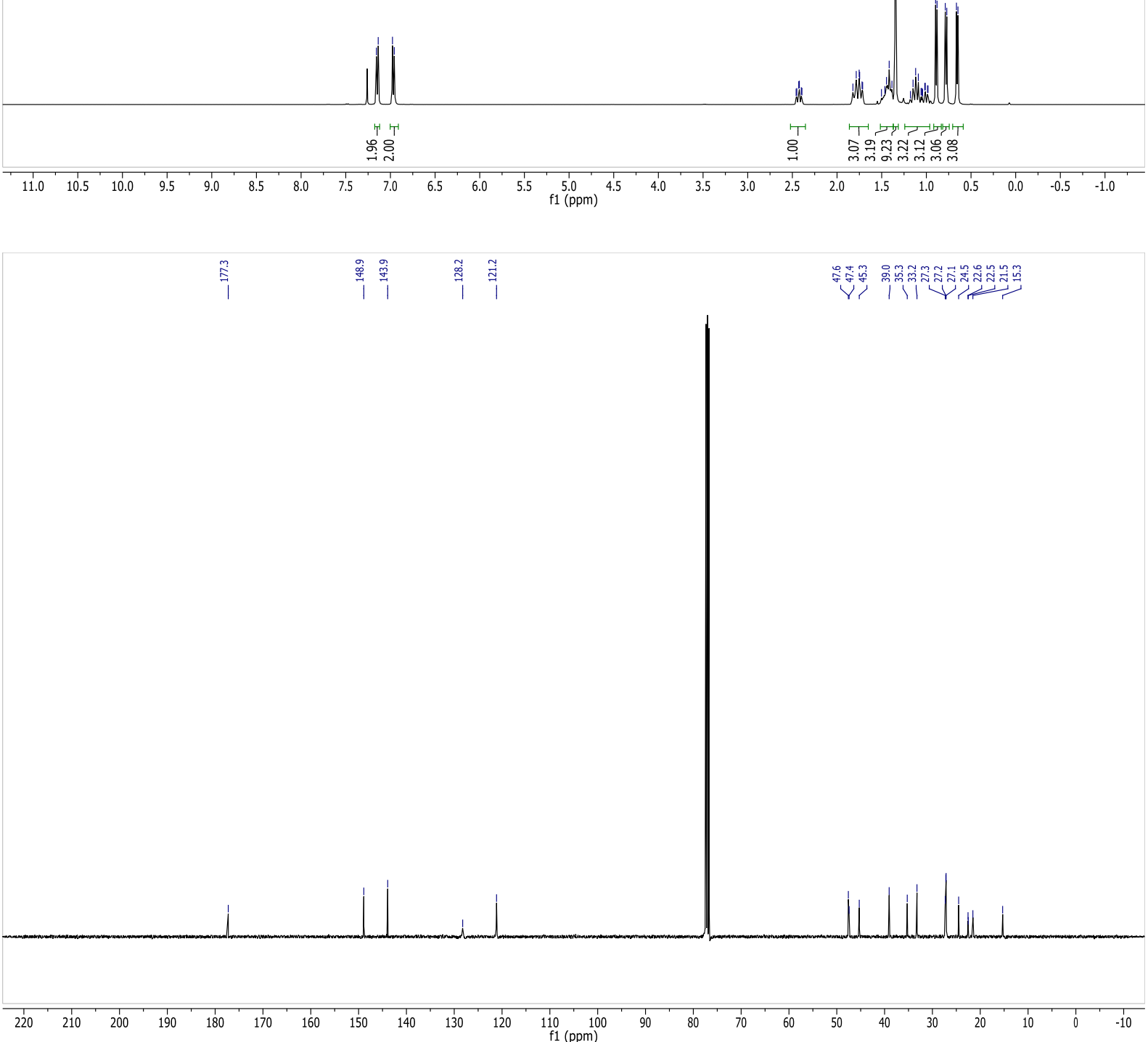
N,N-Diethyl-0-(4-((1R,2S,5R)-2-isopropyl-5-methylcyclohexyl)benzoyl)hydroxylamine (4f)
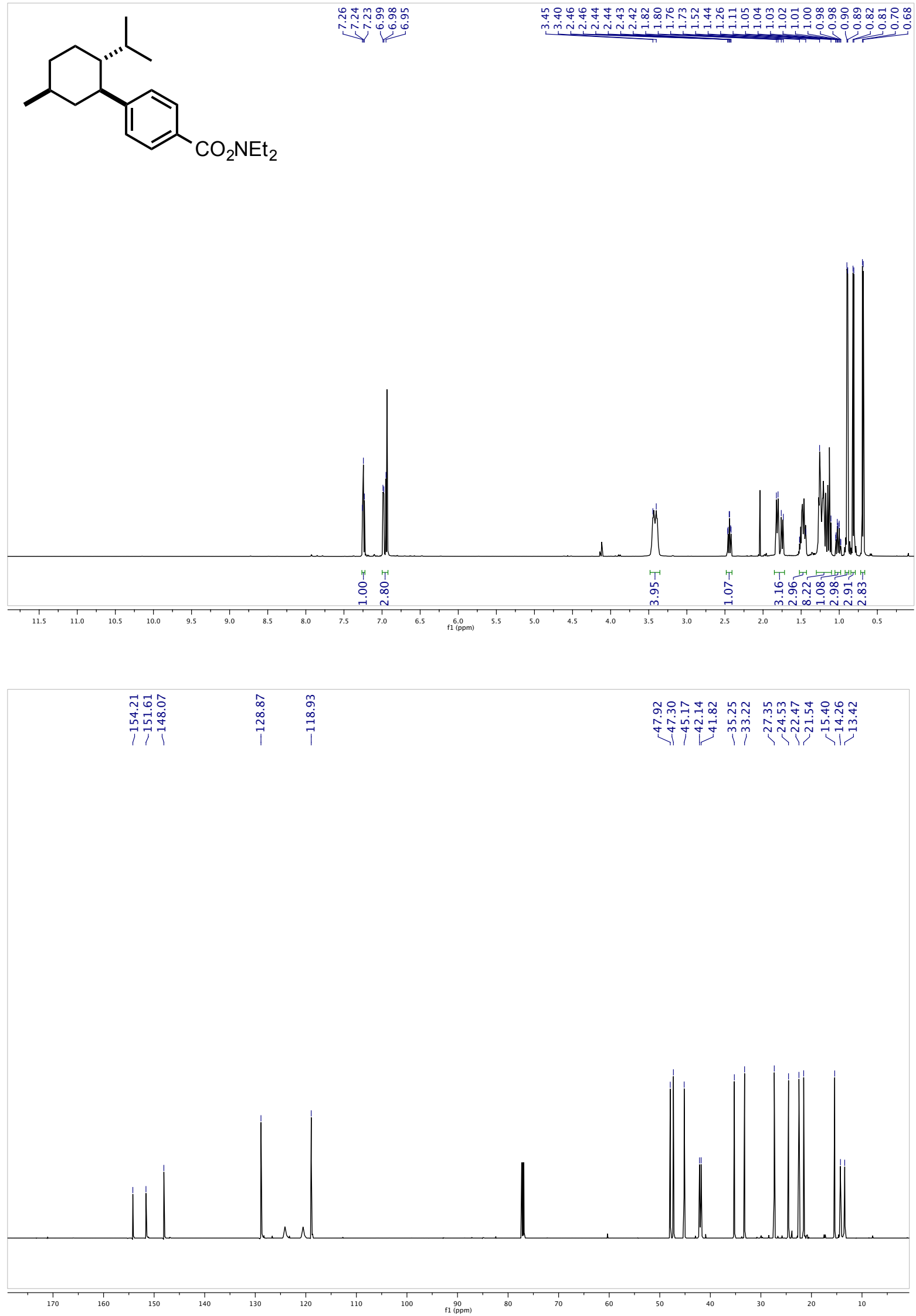
Pentafluoro(3-((1S,2R,5S)-2-isopropyl-5-methylcyclohexyl)phenyl)- $\lambda^{6}$-sulfane (4g)
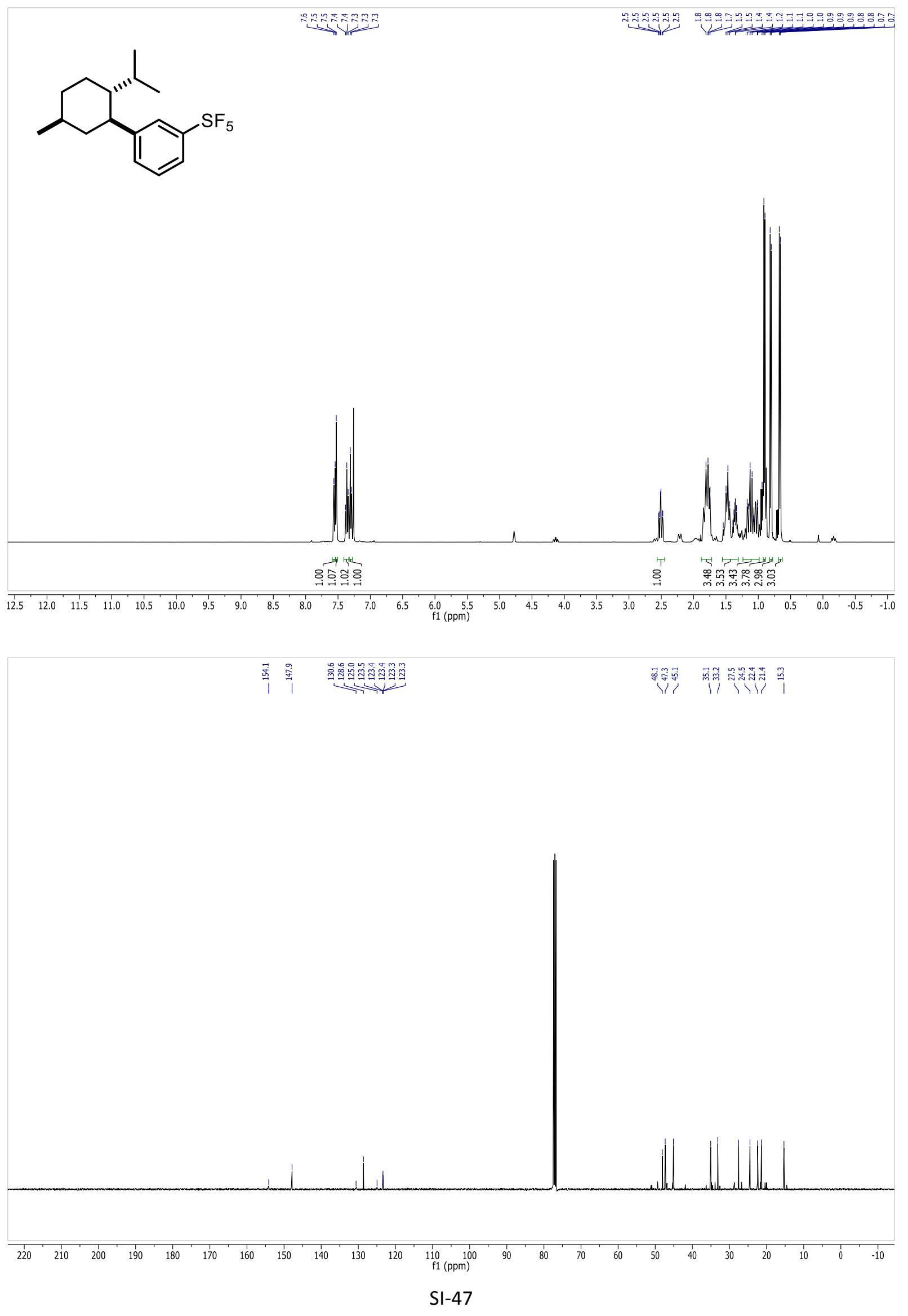
tert-Butyl(3-((1R,2S,5R)-2-isopropyl-5-methylcyclohexyl)phenoxy)dimethylsilane (4h)

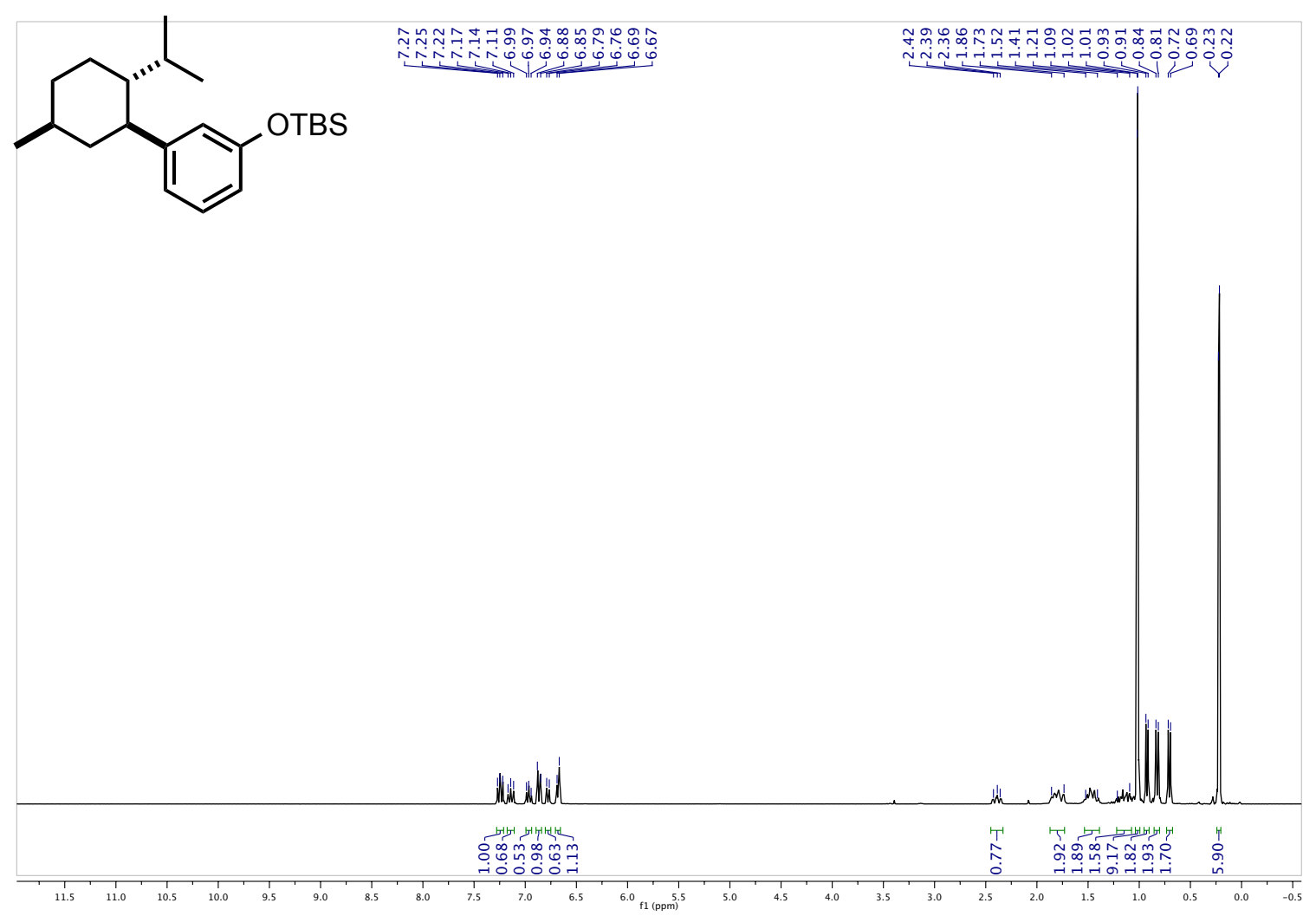

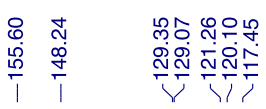
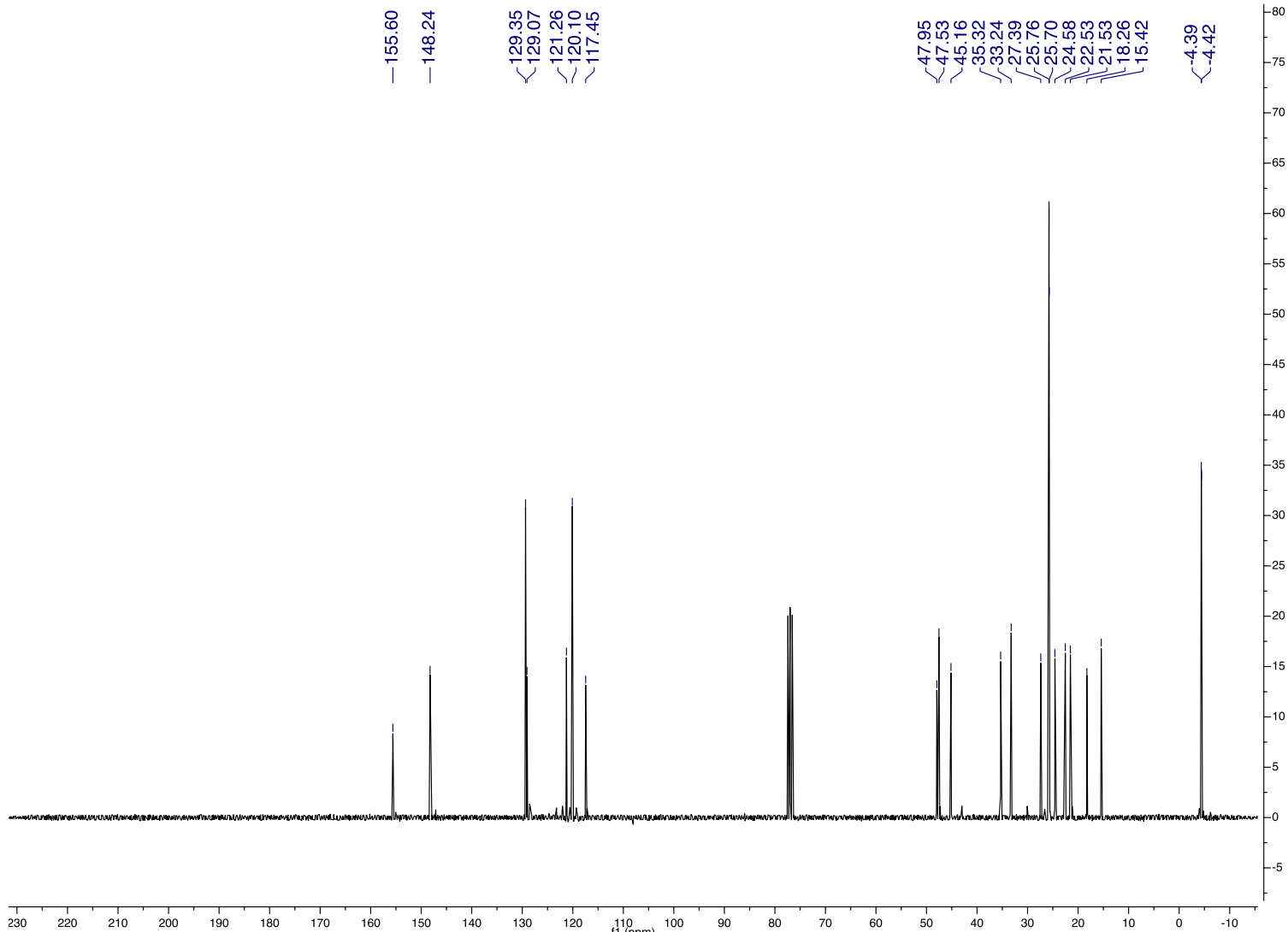
1-((1R,2S,5R)-2-Isopropyl-5-methylcyclohexyl)naphthalene (4i)
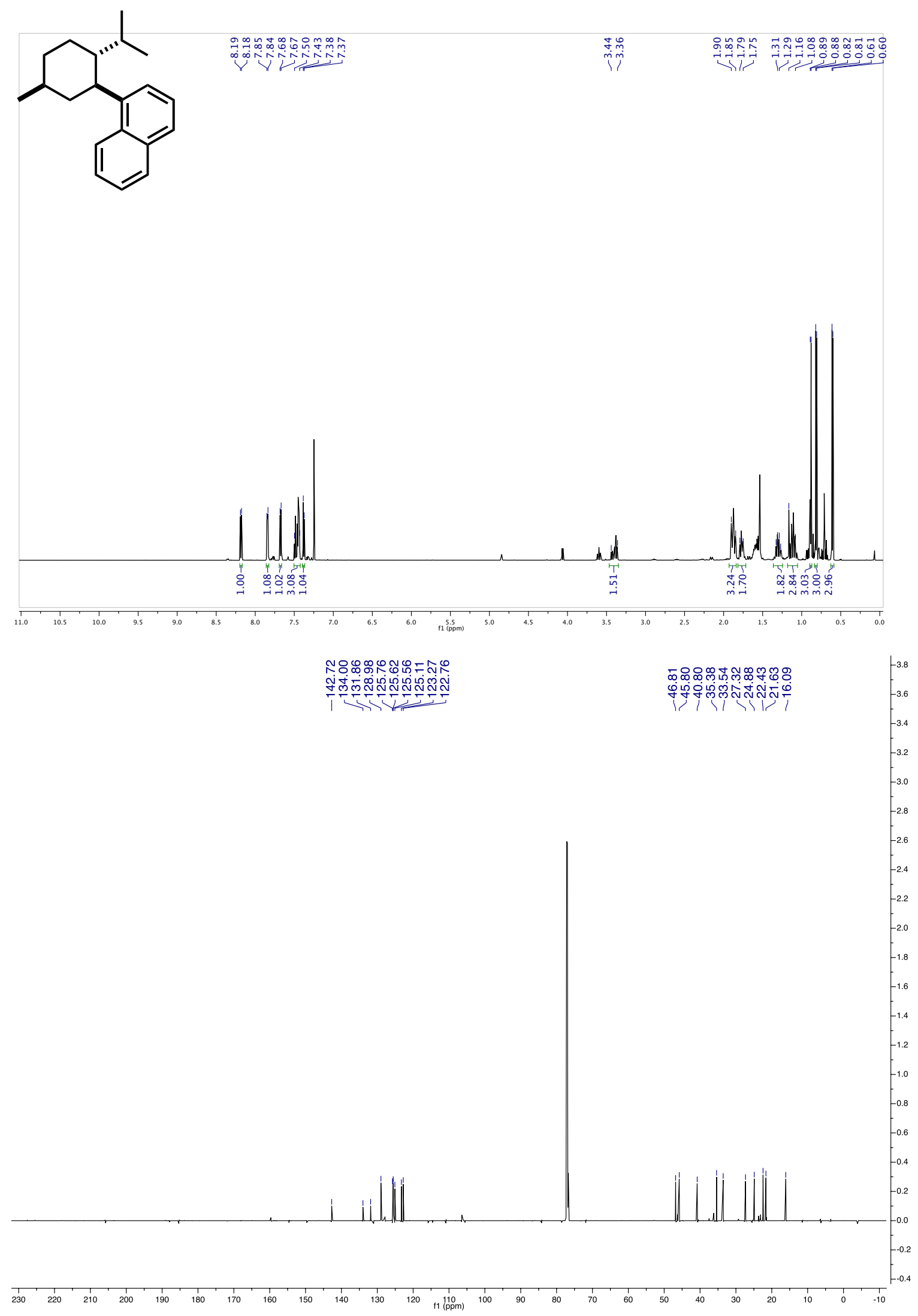
5-((1S,2R,5S)-2-isopropyl-5-methylcyclohexyl)-1-methyl-1H-indole (4j)

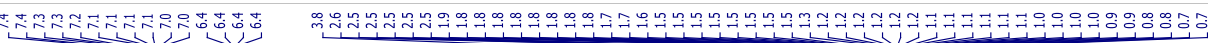<smiles>CC1CC[C@H](C(C)C)C(c2ccc3c(ccn3C)c2)C1</smiles>

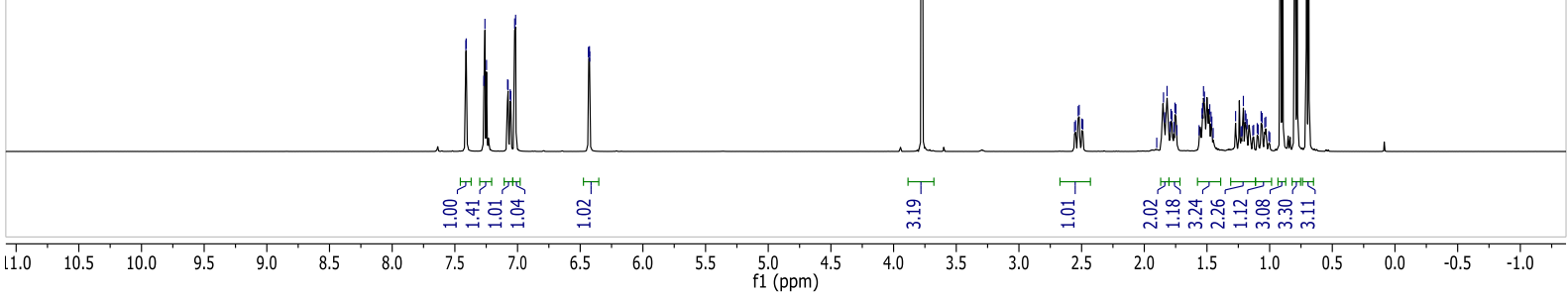

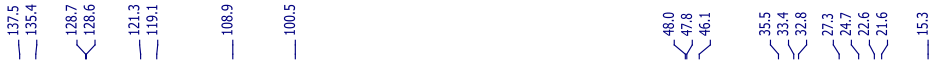

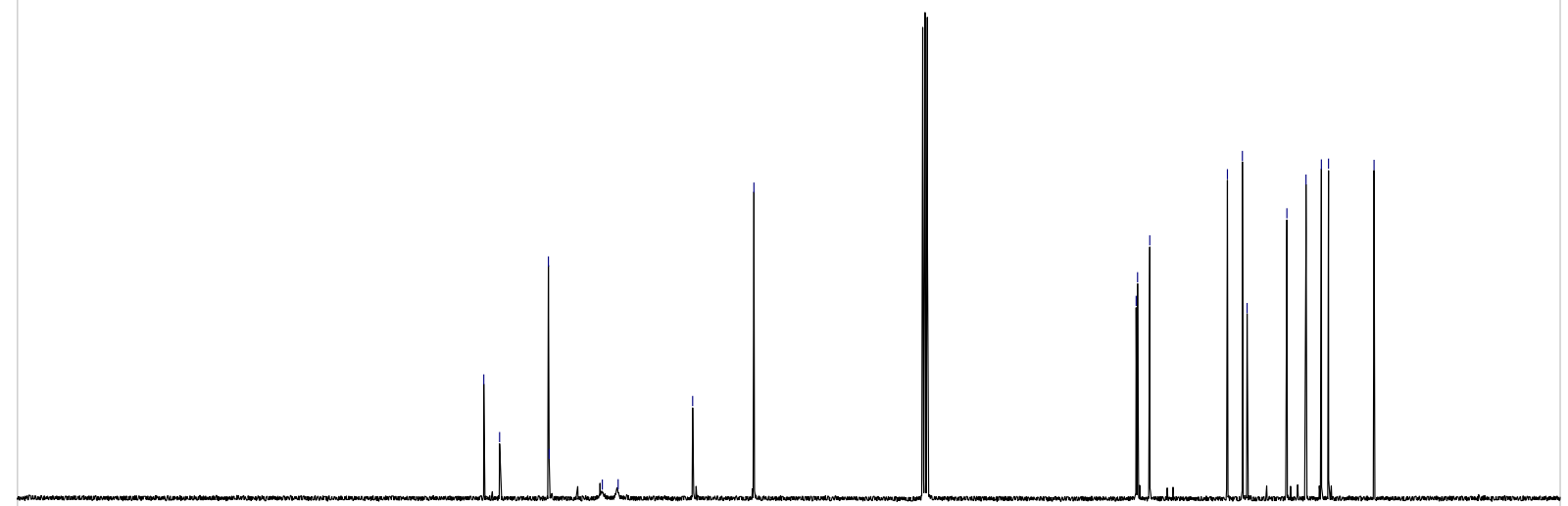

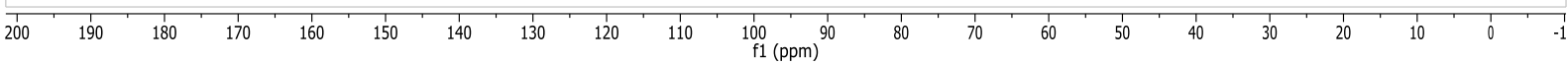


2-((1R,2R)-2-(4-Methoxyphenyl)cyclohexyl)-5,5-dimethyl-1,3-dioxane (5a)
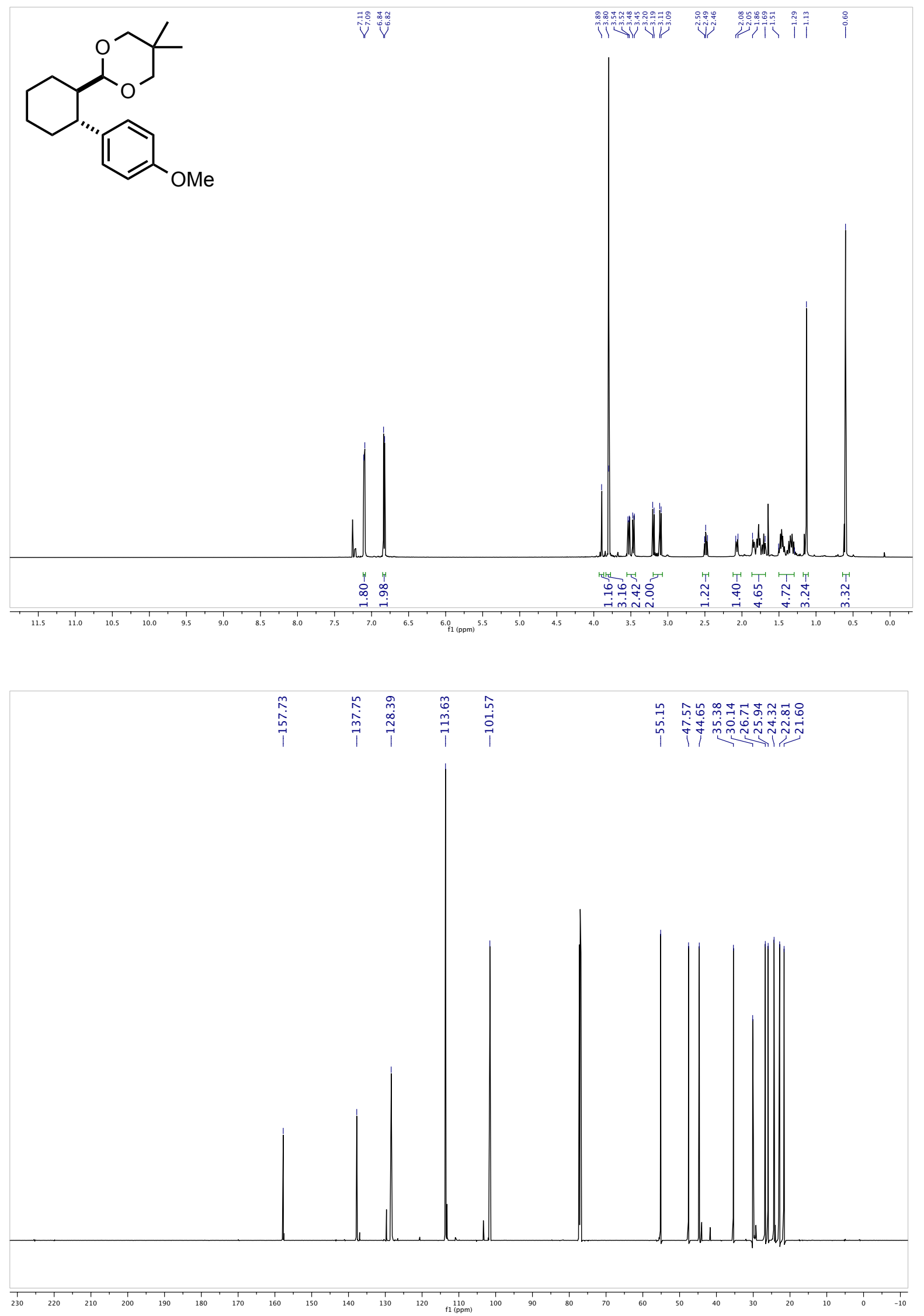

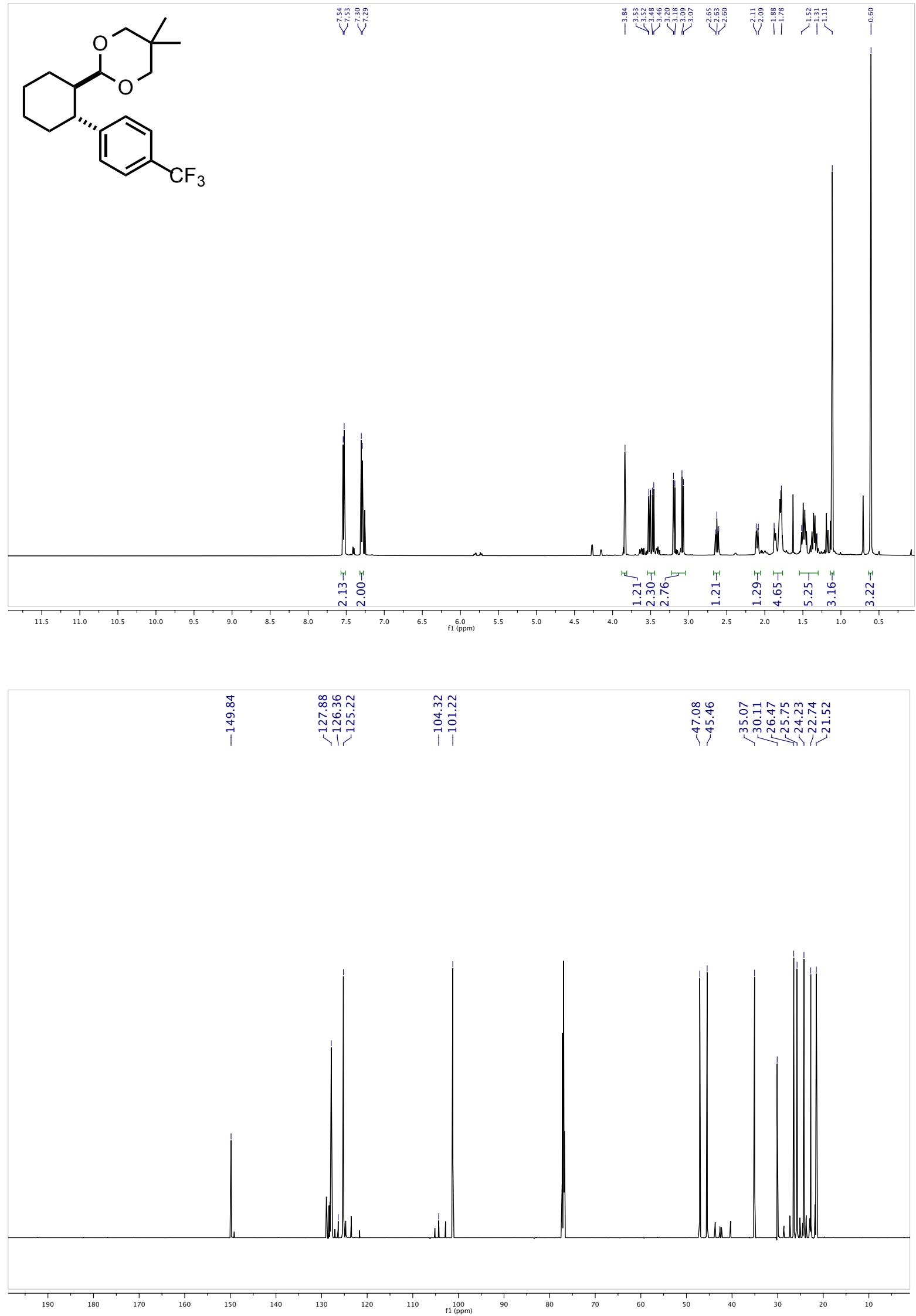
tert-Butyldimethyl(3-((1R,2R,5R)-5-methyl-2-(prop-1-en-2-yl)cyclohexyl)phenoxy)silane (6a)<smiles>C=C(C)[C@H]1CCC(C)CC1c1cccc([18OH])c1</smiles>

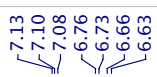

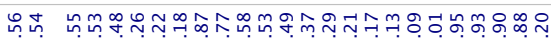

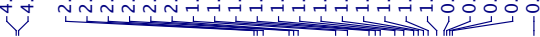
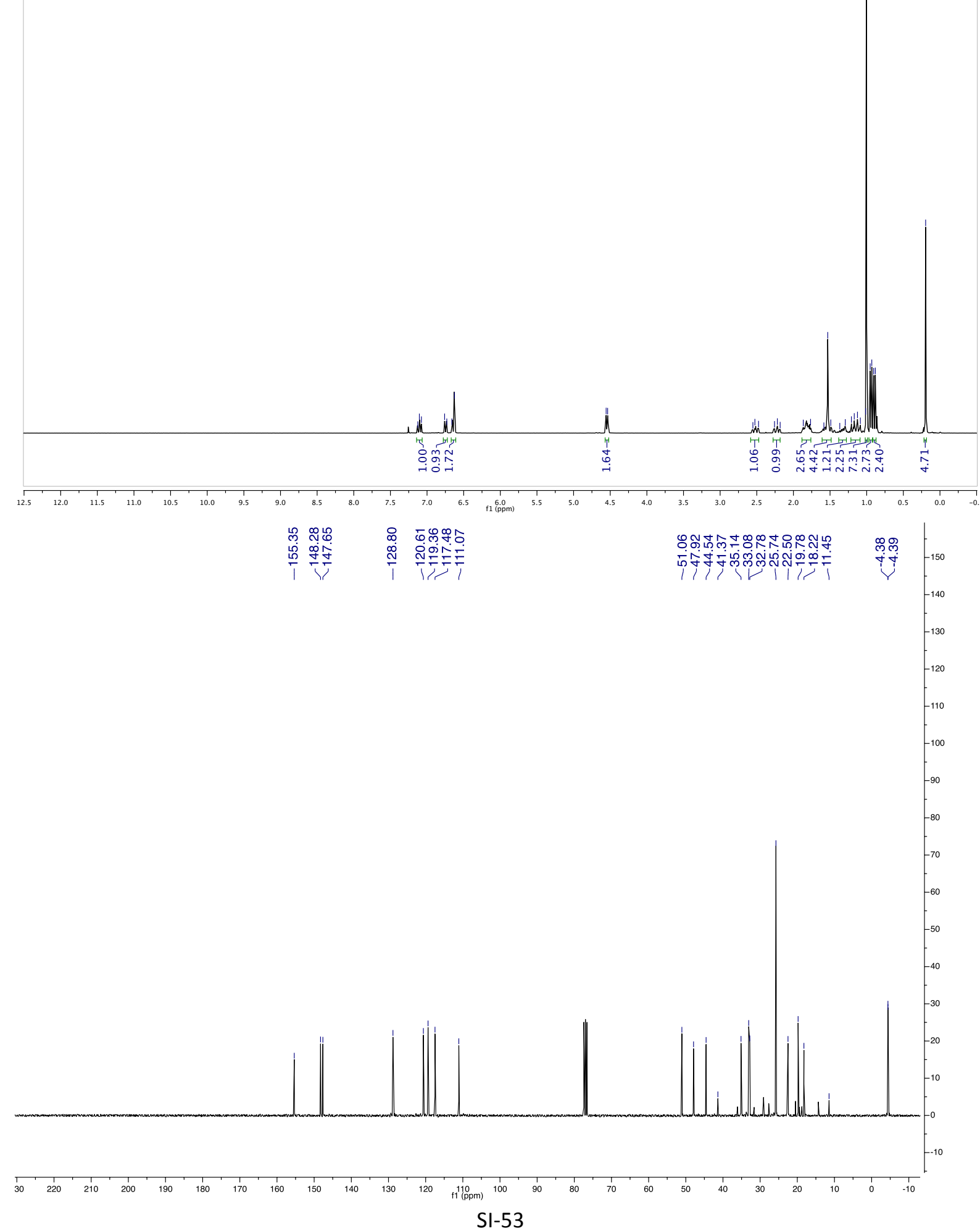
1-((1R,2R,5R)-5-Methyl-2-(prop-1-en-2-yl)cyclohexyl)-4-(trifluoromethyl)benzene (6b)
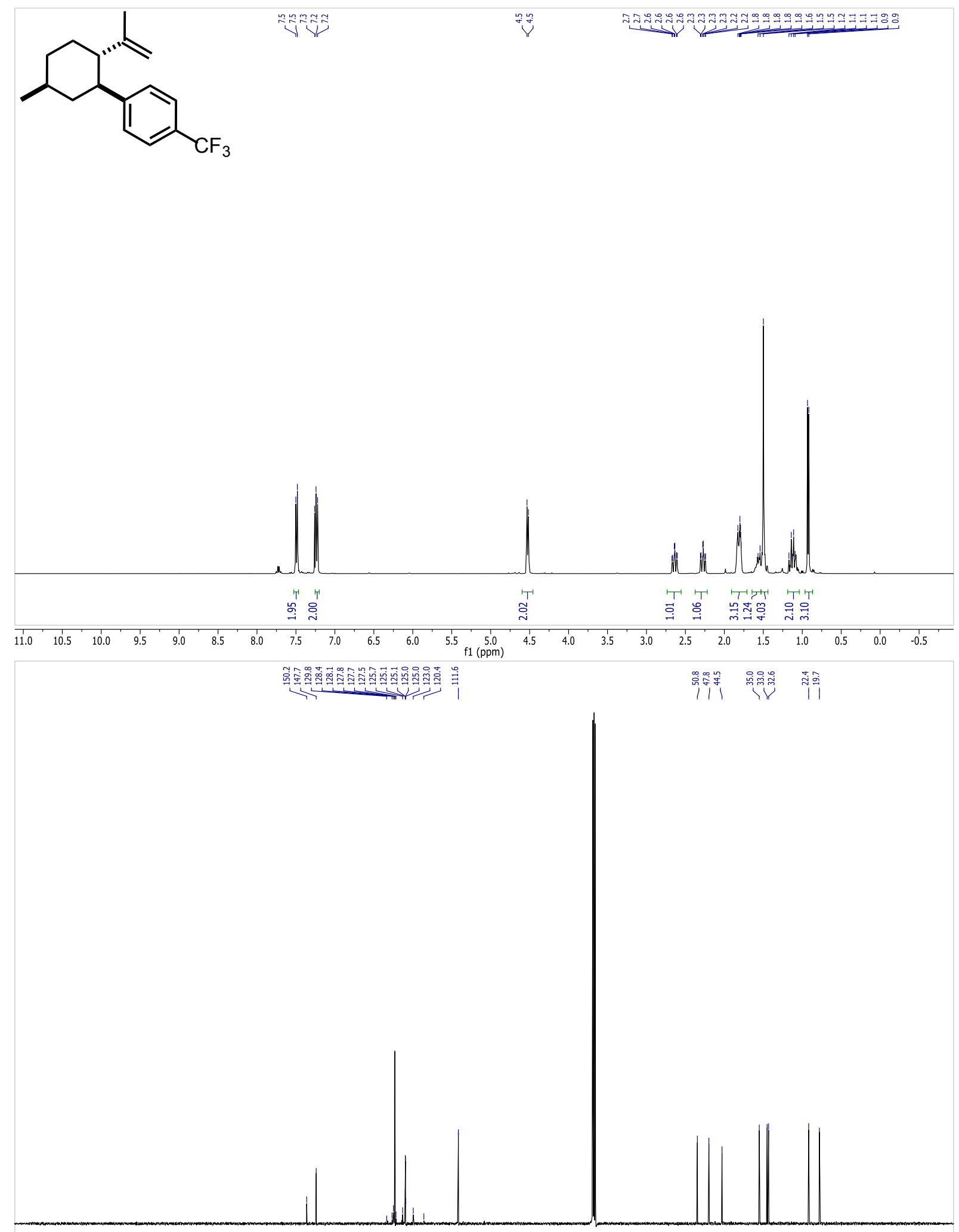

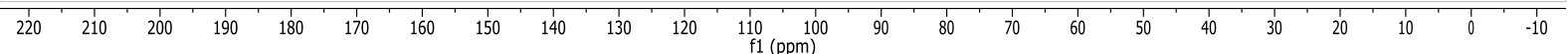


1-Methoxy-4-((1R,2R,5R)-5-methyl-2-(prop-1-en-2-yl)cyclohexyl)benzene (6c)

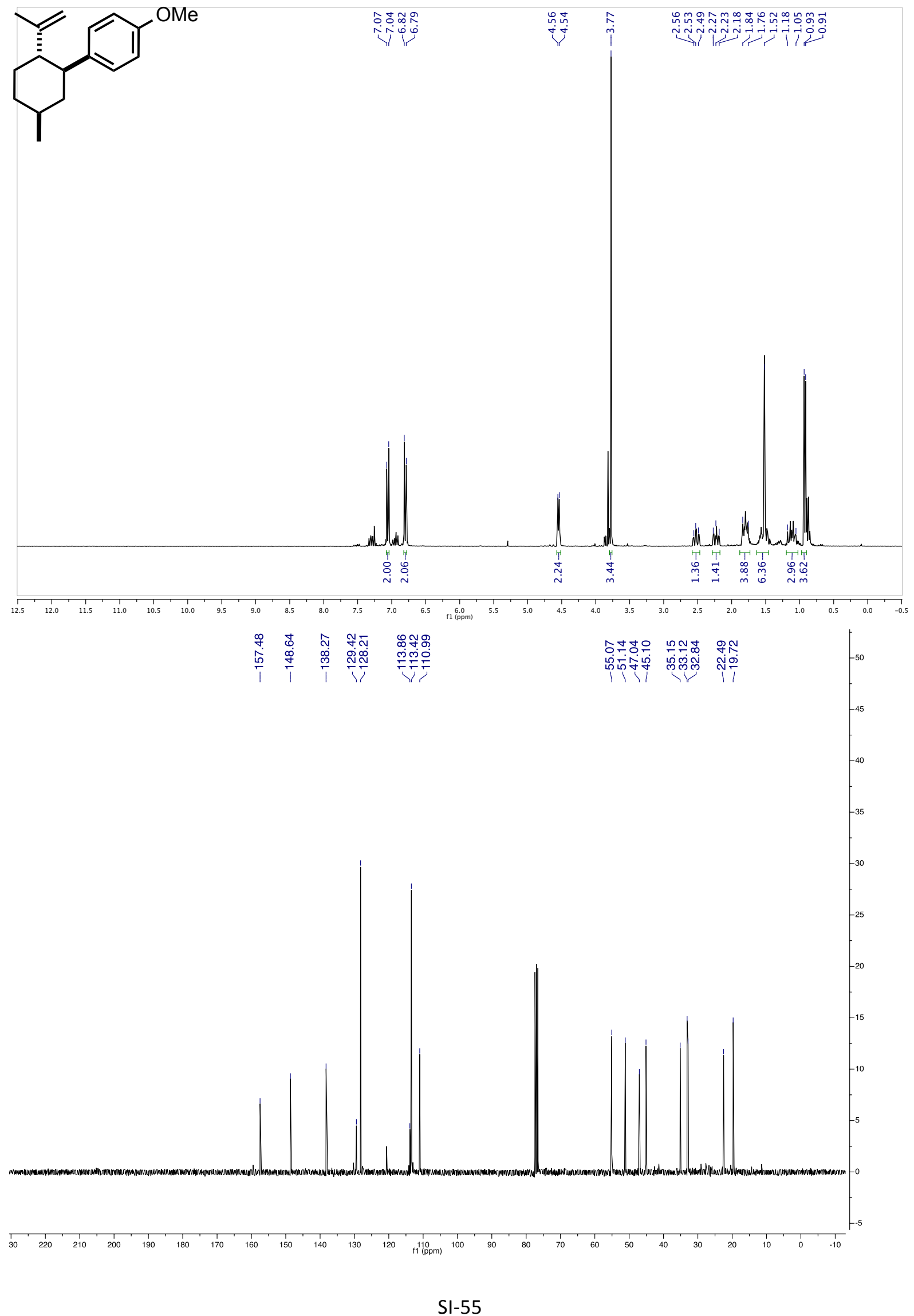


1-((1S,2S,4S)-2-(4-methoxyphenyl)-4-methylcyclohexyl)ethan-1-one (7)
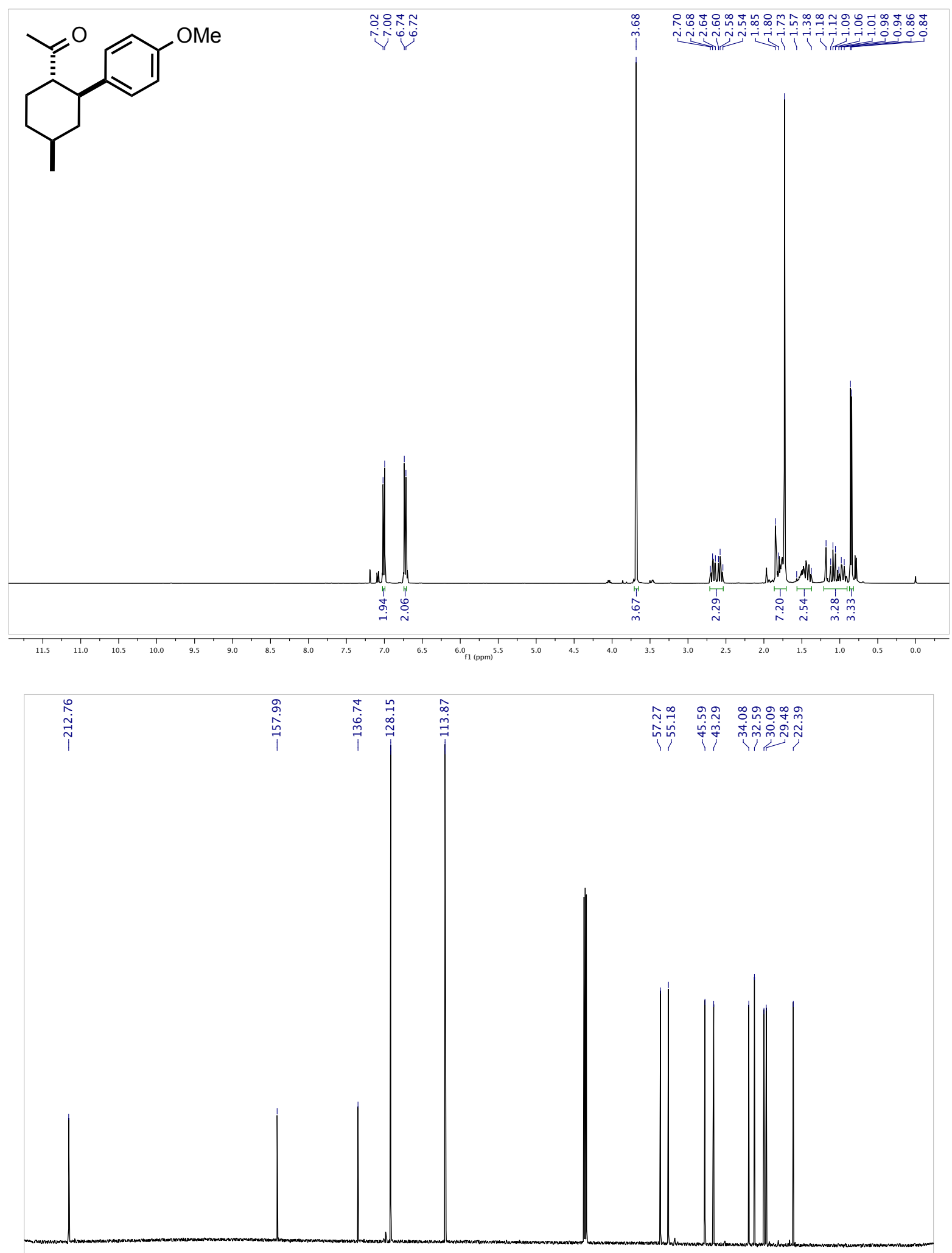
(3S,4R)-3-Isopropyl-4-(4-(trifluoromethyl)phenyl)tetrahydrofuran (8a)
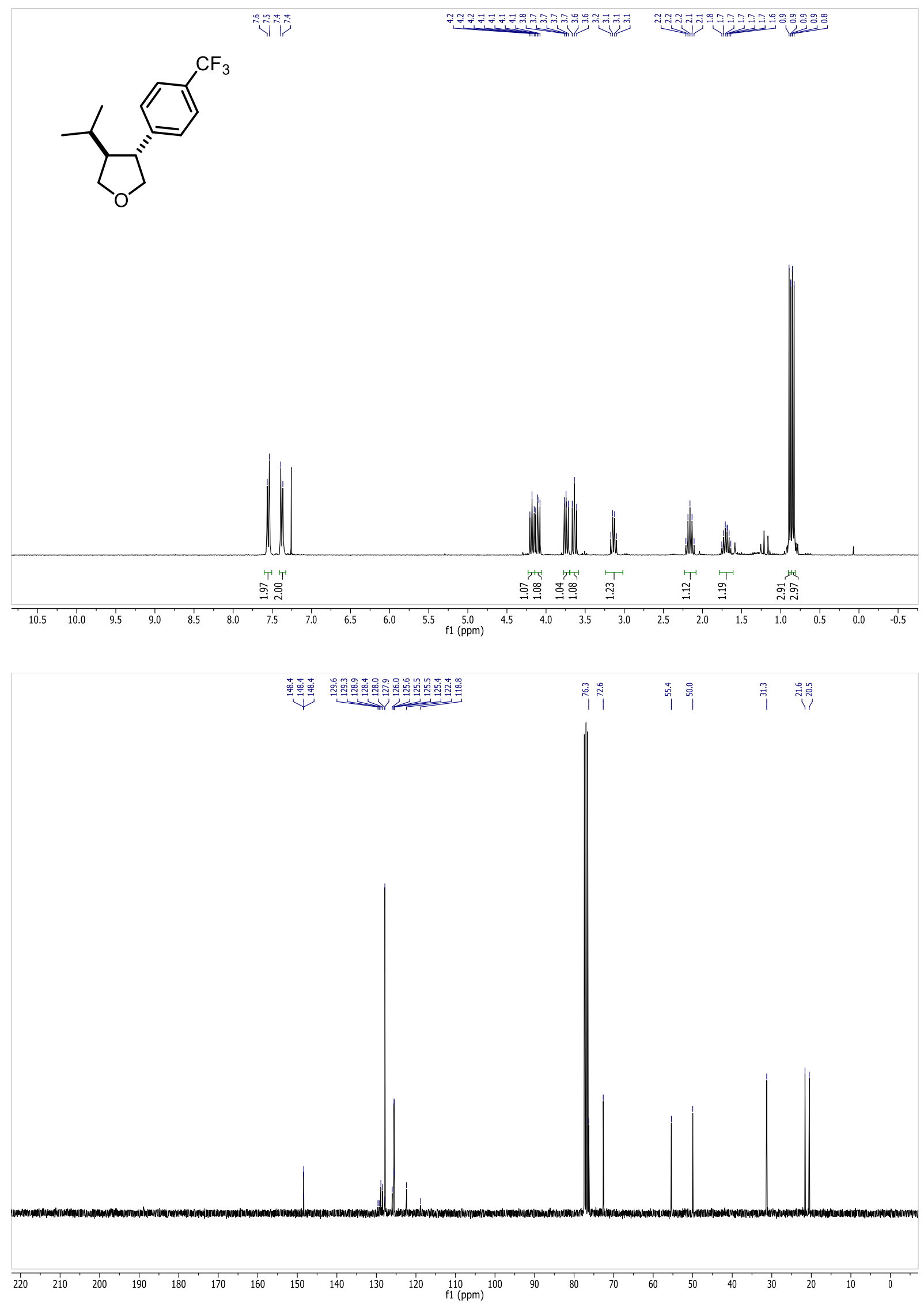
Ethyl 3-((3R,4S)-4-isopropyltetrahydrofuran-3-yl)benzoate (8b)

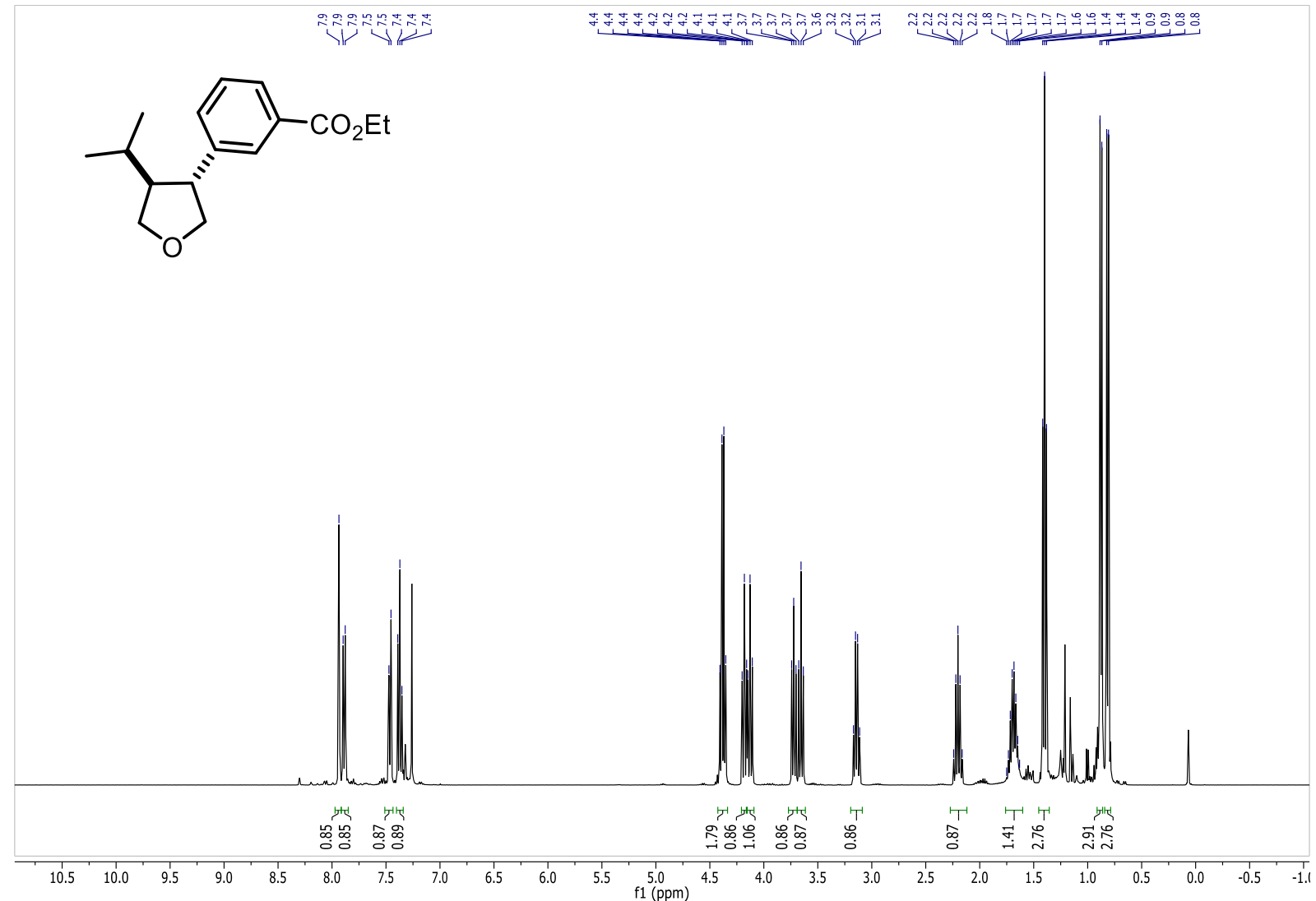

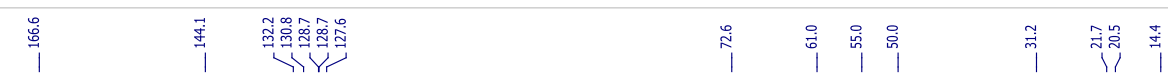

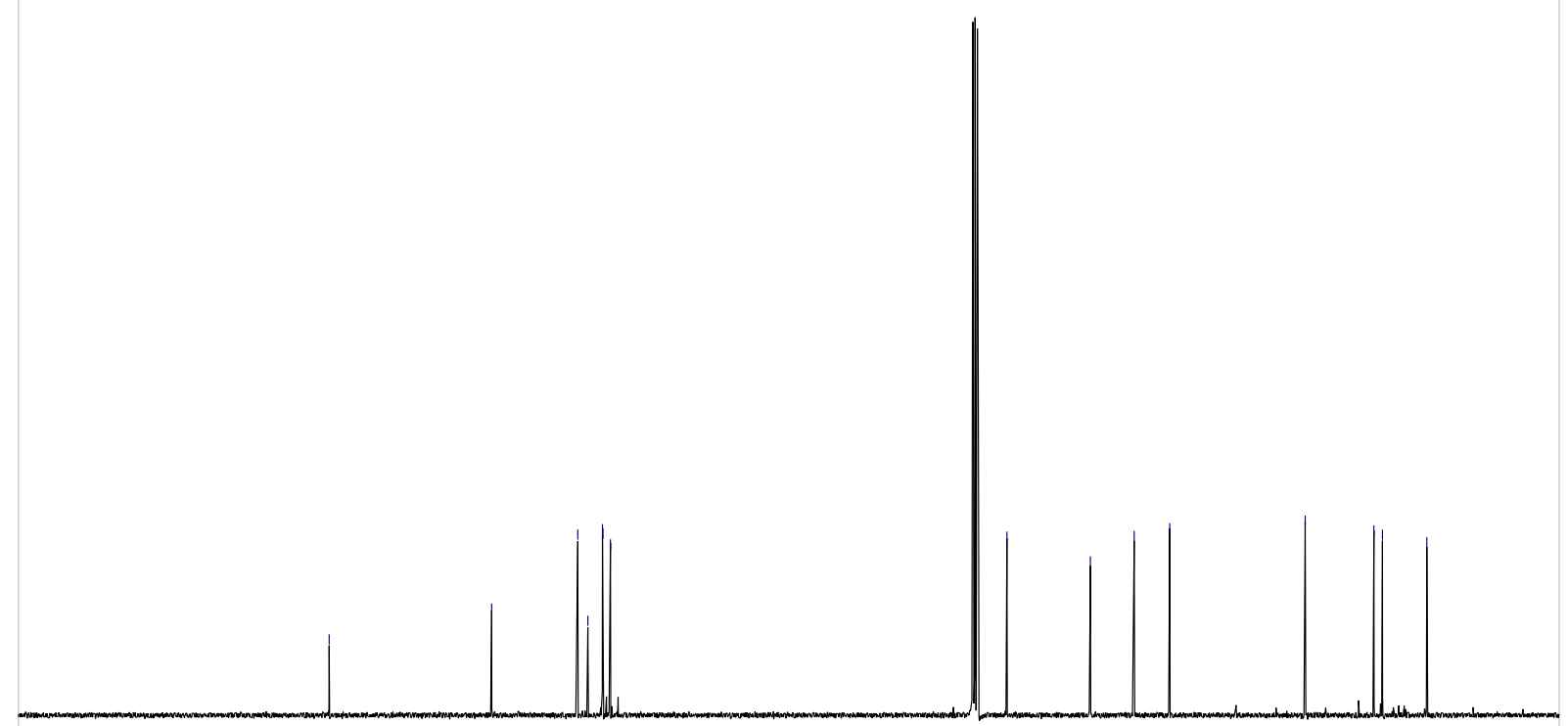

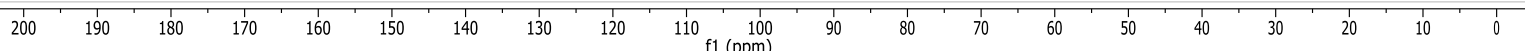


(3R,4R)-3-(Prop-1-en-2-yl)-4-(4-(trifluoromethyl)phenyl)tetrahydrofuran (8c)
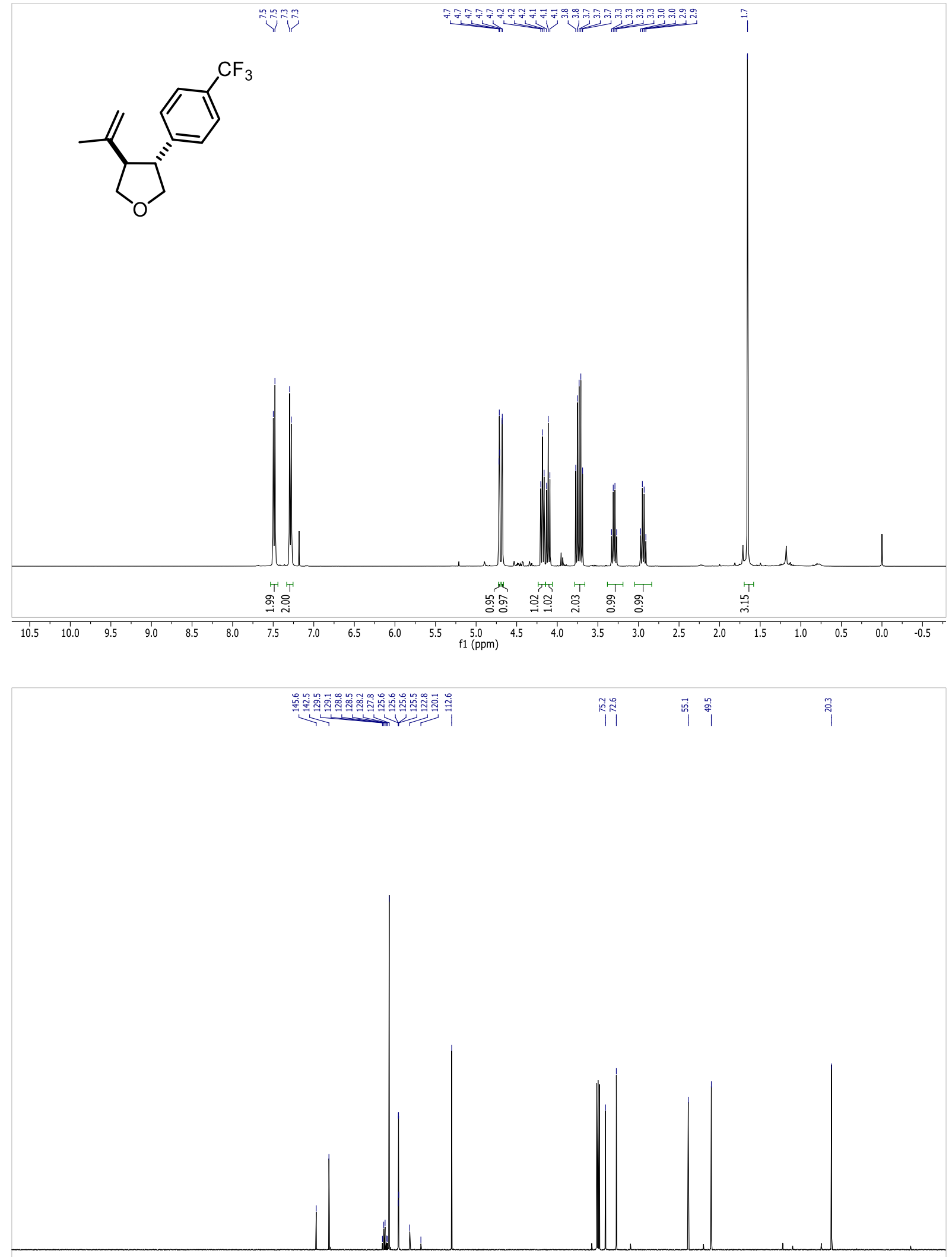

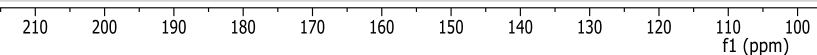


(3R,4R)-3-(4-Methoxyphenyl)-4-(prop-1-en-2-yl)tetrahydrofuran (8d)
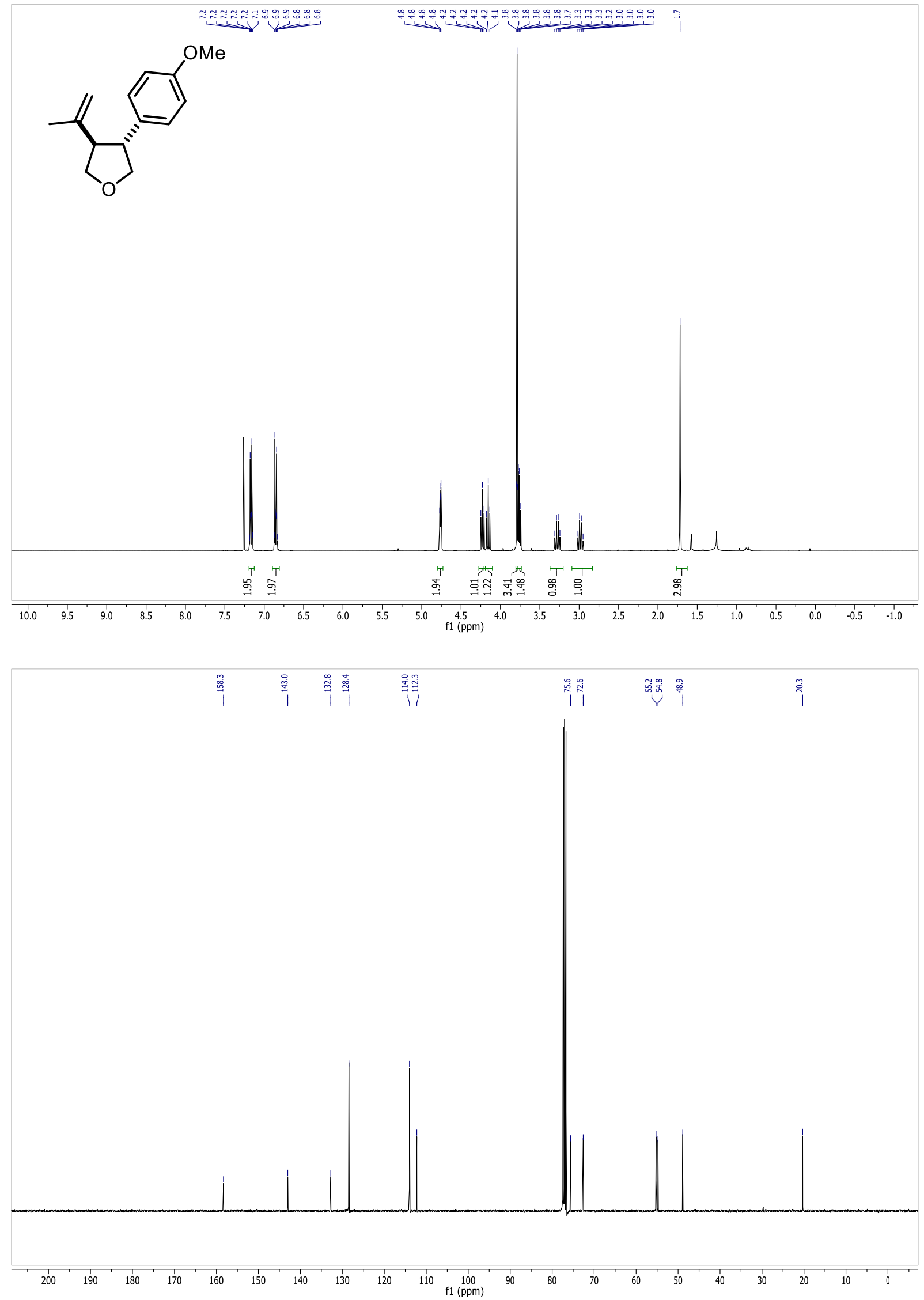
trans-3-Isopropyl-4-(3-methoxyphenyl)-1-tosylpyrrolidine (8e)
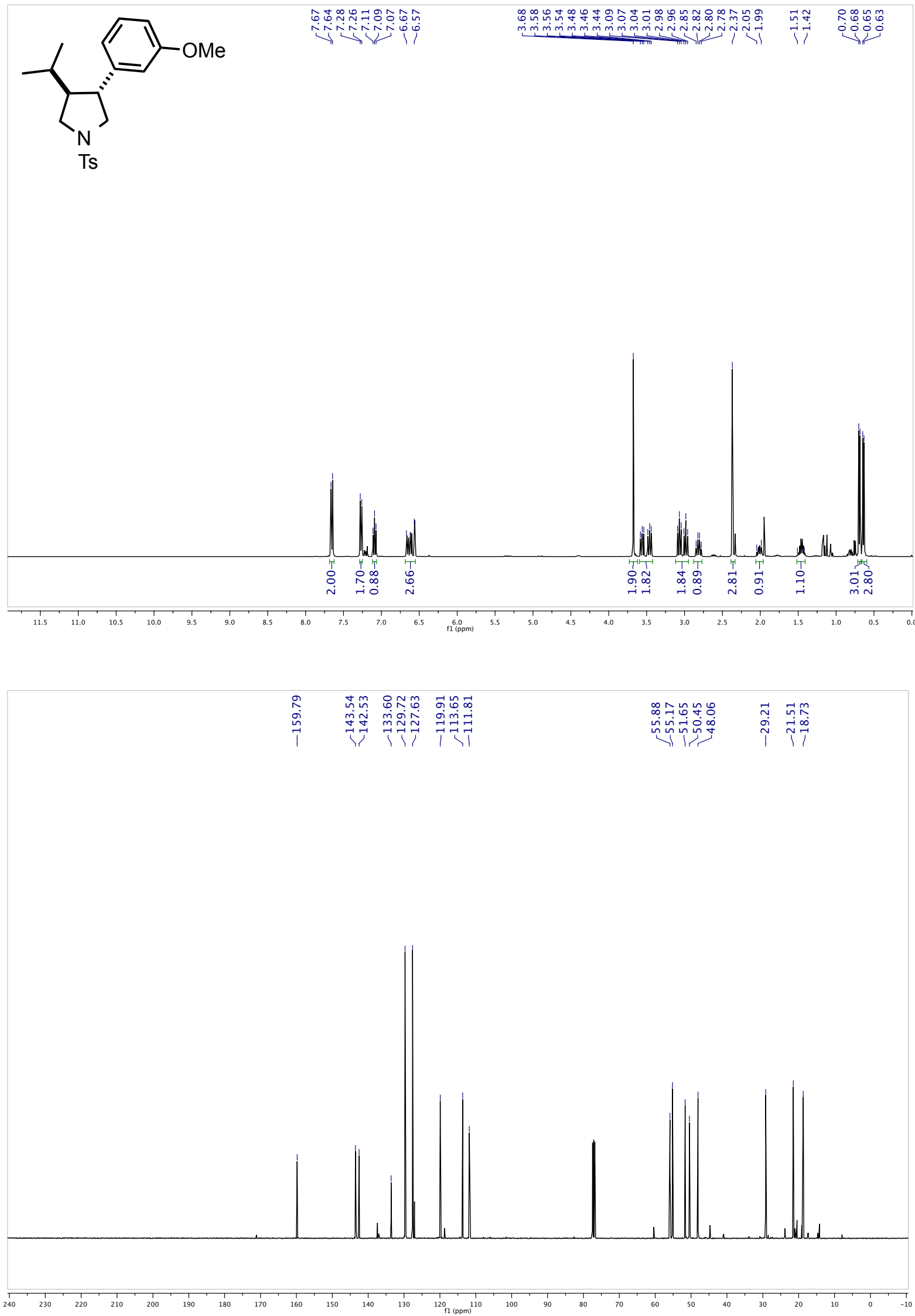
trans-3-Isopropyl-4-(3-methoxyphenyl)-1-tosylpyrrolidine (8f)

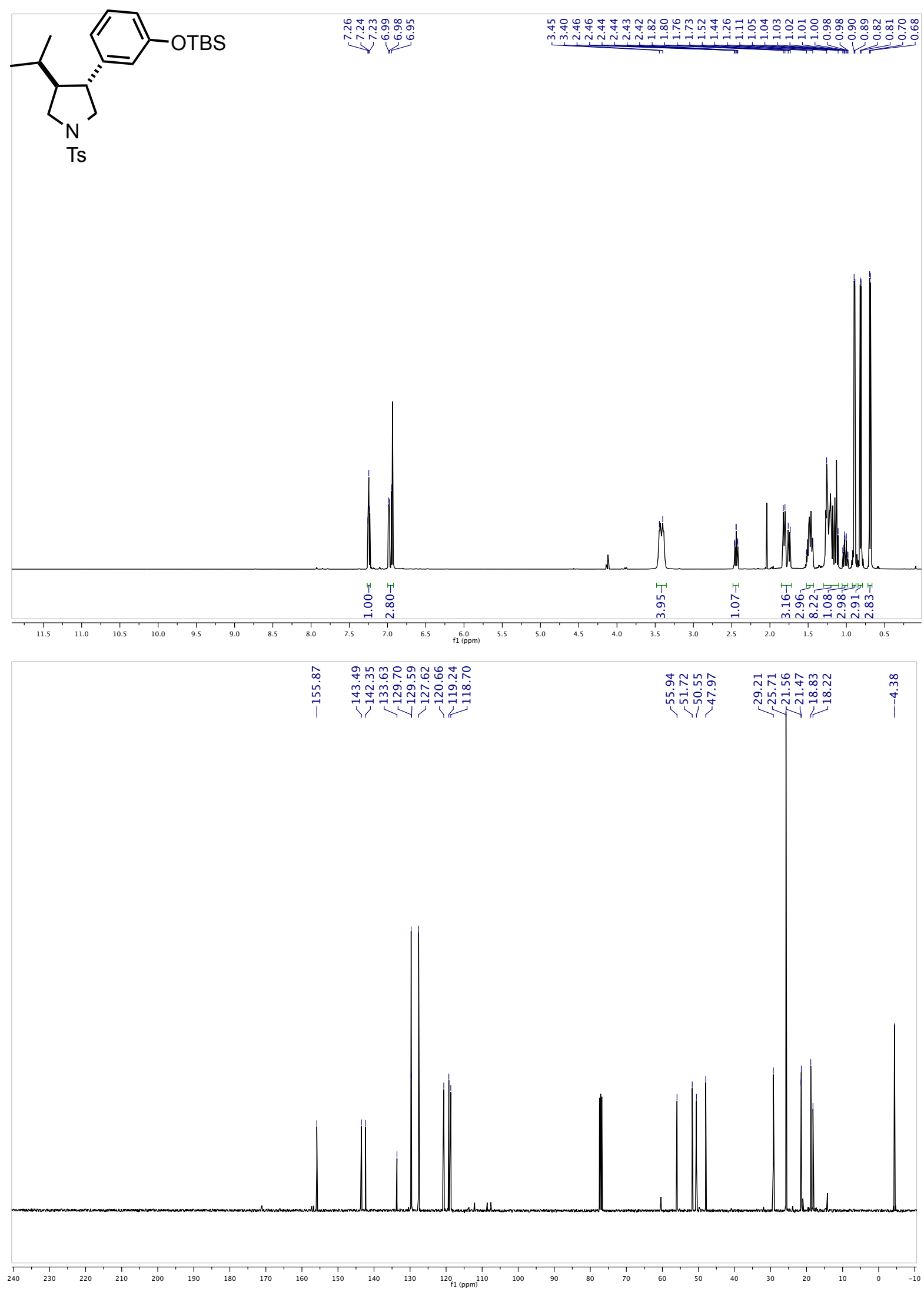

